\title{
On the Semisolvability of Semisimple Hopf Algebras of Low Dimension
}

\author{
Sonia Natale
}

Author address:

Facultad de Matemática, Astronomía y Física, UniverSidad Nacional de Córdoba, CIEM-CONICET, Ciudad Universitaria, (5000) Córdoba, Argentina

E-mail address: natale@mate.uncor.edu

URL: http://www.mate.uncor.edu/natale 


\section{Contents}

Introduction and Main Results 1

$\begin{array}{ll}\text { Conventions and Notation. } & 7\end{array}$

Chapter 1. Semisimple Hopf Algebras 8

1.1. Algebra structure $\quad 8$

1.2. Irreducible characters 9

1.3. Coinvariants of Hopf algebra maps 11

1.4. Yetter-Drinfeld modules $\quad 12$

1.5. Yetter-Drinfeld modules and the character algebra 13

1.6. One dimensional Yetter-Drinfeld modules 14

$\begin{array}{lll}\text { 1.7. } H^{\operatorname{co} B} \text { as a left coideal of } H & 15\end{array}$

Chapter 2. The Nichols-Richmond Theorem 16

2.1. Irreducible characters of degree $2 \quad 16$

2.2. The Nichols-Richmond theorem 18

2.3. An application to $D(H) \quad 19$

2.4. Existence of proper Hopf subalgebras 19

2.5. Hopf subalgebras of index $3 \quad 21$

Chapter 3. Quotient Coalgebras 23

3.1. A multiplicity formula 23

3.2. Stable subcoalgebras $\quad 25$

3.3. Quotients modulo group-like Hopf subalgebras 28

$\begin{array}{ll}\text { 3.4. On the structure of } G(H) & 29\end{array}$

3.5. A criterion of normality 30

Chapter 4. Braided Hopf Algebras 31

4.1. Radford-Majid biproduct construction 31

4.2. Coalgebra structure of $R \quad 32$

4.3. Hopf subalgebras 34

4.4. Biproducts over finite groups 35

4.5. Cocommutative braided Hopf algebras 37

4.6. Cocommutative braided Hopf algebras over $\mathbb{Z}_{p}$

4.7. Transitive actions of central subgroups 41 
Chapter 5. Cocycle Deformations of Some Hopf Algebras 42

5.1. Lifting from abelian groups 42

5.2. Examples in dimension $p q^{2} ; p=1 \bmod q \quad 43$

5.3. Examples in dimension $p q^{2} ; q=1 \bmod p \quad 44$

5.4. Normal Hopf subalgebras in cocycle twists 44

Chapter 6. Dimension $24 \quad 46$

6.1. Possible (co)-algebra structures 46

6.2. Upper and lower semisolvability 51

Chapter 7. Dimension $30 \quad 53$

7.1. Possible (co)-algebra structures 53

7.2. Classification 56

Chapter 8. Dimension $36 \quad 57$

8.1. Reduction of the problem $\quad 57$

8.2. Main result 63

Chapter 9. Dimension $40 \quad 65$

9.1. Reduction of the problem 65

9.2. Main result 69

$\begin{array}{lll}\text { Chapter 10. Dimension } 42 & 70\end{array}$

10.1. Possible (co)-algebra structures $\quad 70$

10.2. Classification $\quad 74$

$\begin{array}{lll}\text { Chapter 11. Dimension } 48 & 75\end{array}$

11.1. First reduction $\quad 75$

11.2. Further reductions $\quad 86$

11.3. Main result up to cocycle twists 90

11.4. Main result 95

Chapter 12. Dimension $54 \quad 100$

12.1. First reduction 100

12.2. Main result 104

Chapter 13. Dimension $56 \quad 105$

13.1. First reduction 105

13.2. Main result 107

Appendix A. Drinfeld Double of $H_{8} \quad 108$

A.1. Structure of $D\left(H_{8}\right) \quad 108$

A.2. Proof of Theorem A.1.1 109

A.3. Proof of Theorem A.1.2 111

$\begin{array}{lll}\text { Appendix. Bibliography } & 112\end{array}$ 


\begin{abstract}
We prove that every semisimple Hopf algebra of dimension less than 60 over an algebraically closed field $k$ of characteristic zero is either upper or lower semisolvable up to a cocycle twist.
\end{abstract}

Received by the editor September 2006.

1991 Mathematics Subject Classification. Primary 16W30; Secondary 17B37.

Key words and phrases. semisimple Hopf algebra; semisolvability; Hopf algebra extension.

This work was partially supported by CONICET, Agencia Córdoba Ciencia, ANPCyT, Fundación Antorchas and Secyt (UNC). 



\section{Introduction and Main Results}

In recent papers several notions and results from the theory of finite groups have been generalized or adapted to the context of (semisimple) Hopf algebras. Simultaneously, many results on the classification of semisimple Hopf algebras have also appeared. Some conjectural analogies, such as Kaplansky's conjecture about the dimensions of the irreducible modules, still remain an open problem.

Let $H$ be a finite dimensional Hopf algebra over a field $k$. A Hopf subalgebra $A \subseteq H$ is called normal if $h_{1} A \mathcal{S}\left(h_{2}\right) \subseteq A$, for all $h \in H$. If $H$ does not contain proper normal Hopf subalgebras then it is called simple.

If $A \subseteq H$ is a normal Hopf subalgebra then the structure of $H$ can be reconstructed from $A$ and the quotient Hopf algebra $\bar{H}=H / H A^{+}$; more precisely, it is known that in this case $H$ is isomorphic to a bicrossed product $H \simeq A \#_{\rightarrow, \sigma}^{\rho, \tau} \bar{H}$, where $(\rightarrow, \sigma, \rho, \tau)$ is a compatible datum; see for instance $[\mathbf{A}, \mathbf{M}, \mathbf{M} \mathbf{1 0}]$. This fact implies that, when trying to classify Hopf algebras of a given finite dimension, it is an important problem to decide whether the Hopf algebra is simple or not.

We shall assume from now on that the field $k$ is algebraically closed of characteristic zero.

We say that a finite dimensional Hopf algebra $H$ is trivial if it is isomorphic to a group algebra or to a dual group algebra. Then, $H$ is trivial if and only if it is commutative or cocommutative.

The notions of upper and lower semisolvability for finite-dimensional Hopf algebras have been introduced in $[\mathbf{M W}]$, as generalizations of the notion of solvability for finite groups. By definition, $H$ is called lower semisolvable if there exists a chain of Hopf subalgebras $H_{n+1}=k \subseteq$ $H_{n} \subseteq \cdots \subseteq H_{1}=H$ such that $H_{i+1}$ is a normal Hopf subalgebra of $H_{i}$, for all $i$, and all factors $\bar{H}_{i}:=H_{i+1} / H_{i+1} H_{i}^{+}$are trivial. Dually, $H$ is called upper semisolvable if there exists a chain of quotient Hopf algebras $H_{(0)}=H \rightarrow H_{(1)} \rightarrow \cdots \rightarrow H_{(n)}=k$ such that each of the maps $H_{(i-1)} \rightarrow H_{(i)}$ is normal, and all factors $H_{i}:=H_{(i-1)}^{\mathrm{co} \pi_{i}}$ are trivial; here, $H_{(i-1)}^{\mathrm{co} \pi_{i}}$ is the space of coinvariants of the map $\pi_{i}$, see Section 1.3. 
We have that $H$ is upper semisolvable if and only if $H^{*}$ is lower semisolvable $[\mathbf{M W}]$. If this is the case, then $H$ can be obtained from group algebras and their duals by means of (a finite number of) extensions; in particular, $H$ is semisimple.

The smallest non-solvable group is the simple alternating group $\mathbb{A}_{5}$ of order 60. It is thus natural to ask if an analogous statement is true for semisimple Hopf algebras. A version of the following question was posed by S. Montgomery in [Mo1, Question, pp. 269].

QUESTION 1. Let $H$ be a semisimple Hopf algebra of dimension less than 60. Is $H$ necessarily upper or lower semisolvable?

Let $H$ be a semisimple Hopf algebra over $k$. If $\operatorname{dim} H=p^{n}$, where $p$ is a prime number, then $H$ has a nontrivial central group-like element [M6]; inductively, one can see that $H$ is both upper and lower semisolvable [MW]. Also, if $\operatorname{dim} H=p q^{2}$, where $p \neq q$ are prime numbers, then it was shown in $[\mathbf{N}, \mathbf{N} 2, \mathbf{N} 3]$ that, under the assumption that $H$ and $H^{*}$ are both of Frobenius type, either $H$ or $H^{*}$ contains a nontrivial central group-like element. This implies that these Hopf algebras are also semisolvable, since semisimple Hopf algebras of dimension $p$, $p q$ and $q^{2}$ are trivial. In [N3] we showed that all semisimple Hopf algebras of dimension $p q^{2}<100$ are of Frobenius type (some instances of this fact, e.g., dimension 44, appeared in [K2]); so that these are all semisolvable.

However, not every nontrivial semisimple Hopf algebra $H$ is semisolvable. An example of a simple nontrivial semisimple Hopf algebra $H$ of dimension 60 and was constructed by D. Nikshych in $[\mathbf{N k}]$ : in this case $H$ is a cocycle twist of the group algebra of the simple group $\mathbb{A}_{5}$. Moreover, it was shown in $[\mathbf{N k}]$ that if $G$ is a finite simple group and $\phi \in k G \otimes k G$ is a nontrivial invertible pseudo 2-cocycle, then the twisted group algebra $(k G)_{\phi}$ is a nontrivial semisimple Hopf algebra, which is simple as a Hopf algebra.

The smallest example of a semisimple Hopf algebra which is not semisolvable is a cocycle twist of a group of order $36[\mathbf{G N}]$. So the answer to Question 1 is negative, and it can only be expected to be affirmative up to a cocycle twist. The dimensions where the problem remains open are $24,30,36,40,42,48,54$ and 56 . We refer the reader to $[\mathbf{A 2}$, Mo1] for an account of previous results on the problem of classification.

We also point out that, in the related context of Kac algebras, several classification results in low dimension were obtained by Izumi and Kosaki in their work $[\mathbf{I K}]$; in that paper, the authors classify all Kac algebras of dimensions 16, 24, $p q^{2}<60$ and $p q r<60$. 
Our main result is the following theorem, giving an affirmative answer to Question 1 up to cocycle twists.

TheOREm 1. Let $H$ be a semisimple Hopf algebra of dimension $<60$. Then $H$ is either upper or lower semisolvable up to a cocycle twist.

We prove that a semisimple Hopf algebra of dimension 24, 30, 40, 42, 48, 54 or 56 is not simple, and moreover, in dimension 36 the only simple example is a twisting of a finite group. This is equivalent to the statement in the theorem, in view of previous results. Indeed, if $H$ is a nontrivial semisimple Hopf algebra of dimension $<60$, then the following are equivalent (see $[\mathbf{N 4}]$ ):

- $H$ is not simple;

- $H$ is either upper or lower semisolvable.

Our approach to the problem is the following: for each fixed dimension, we first consider the possible algebra and coalgebra decompositions (which turn out to be of Frobenius type). Then, for each possible type, we derive the existence of proper normal Hopf subalgebras.

In order to do this, we discuss some general result on semisimple Hopf algebras. Many of these results are new. We discuss some properties of irreducible characters of low degree which allow, in most cases, to prove the existence of quotient Hopf algebras or Hopf subalgebras, for each fixed algebra or coalgebra structure, respectively. One of the main tools for this is the use of the Nichols-Richmond theorem on irreducible characters of degree $2[\mathbf{N R}]$ and some of its consequences, which we develope in Chapter 2.

Using the character theory of $H$, we get a general result on Hopf subalgebras with index 3, which is often of use in low dimensions. See Theorem 2.5.1.

In Chapter 3 we consider an inclusion $A \subseteq H$ of semisimple Hopf algebras. We show that if $C$ is a simple subcoalgebra of $H$ such that $C a \subseteq C$, for all $a \in A$, then the dual of the quotient coalgebra $C / C A^{+}$ and the crossed product $A_{\alpha}$, where $\alpha: A \otimes A \rightarrow k$ is a certain 2-cocycle, constitute a commuting pair in $C^{*}$.

This relates the corepresentation theory of $C / C A^{+}$with the representation theory of the Galois object $A_{\alpha}$ of $A$. Applied in combination with Masuoka's main result in [M5] on deformations of cosemisimple Hopf algebras, the result allows us to prove that some coalgebra decompositions are impossible. The result is also useful to get some information on the structure of the Hopf subalgebra $A$, especially when $A$ is a group algebra; see Section 3.4. 
We also discuss braided Hopf algebras in relation with the RadfordMajid biproduct construction; see Chapter 4. It often happens, mainly because of the 'self-dual' nature of the assumption of simplicity, that if a given semisimple Hopf algebra $H$ is simple, then $H$ must have the structure of a biproduct $H=R \# A$, where $A$ is a semisimple Hopf subalgebra. We get several results on existence of proper (normal) Hopf subalgebras in biproducts; in particular, we give such a result in Corollary 4.3 .4 for the case where $A$ is not cocommutative of dimension $p^{3}, p$ a prime number.

Let $p \neq q$ be prime numbers. We describe the known families in dimension $p q^{2}$ as cocycle twists of group algebras; we also prove that other families cannot be obtained in this fashion. See Chapter 5.

The classification of semisimple Hopf algebras of dimension $p q r$, where $p, q$ and $r$ are distinct prime numbers, was given in $[\mathbf{N}]$ under the assumption that $H$ admits an extension with commutative 'kernel' and cocommutative 'cokernel' (a so called abelian extension).

In this paper we also prove that semisimple Hopf algebras of dimension 30 and 42 admit abelian extensions. This allows us to give the complete classification of semisimple Hopf algebras of these dimensions. We obtain the following theorems. See Chapters 7, 10.

THEOREM 2. Let $H$ be a semisimple Hopf algebra of dimension 30 over $k$. Then $H$ is isomorphic to a group algebra $k G$ or to a dual group algebra $k^{G}$, where $G$ is a group of order 30 .

The known nontrivial examples in dimension 42 were constructed in $[\mathbf{A N}]$ : these are denoted $\mathcal{A}_{7}(2,3)$ and $\mathcal{A}_{7}(3,2) \simeq \mathcal{A}_{7}(2,3)^{*}$. We prove in this paper the following result.

THEOREM 3. Let $H$ be a nontrivial semisimple Hopf algebra of dimension 42 over $k$. Then $H$ is isomorphic to one of the Hopf algebras $\mathcal{A}_{7}(2,3)$ or $\mathcal{A}_{7}(3,2)$.

In Table 1 we resume some known facts about the classification of semisimple Hopf algebras of dimension less than 60. In the first column, $p, q$ and $r$ are distinct prime numbers. The table is organized as follows: the first column indicates the factorizations of the dimensions $\leq 60$ in terms of prime numbers; the second and third column contain the references where nontrivial examples were constructed and where the classification was given, respectively. The fourth column gives additional information for each specific type.

We include an appendix where we describe the structure of the Drinfeld doubles of the three non-commutative semisimple Hopf algebras of dimension 8. Tambara and Yamagami show in $[\mathbf{T Y}]$ that the 


\begin{tabular}{|c|c|c|c|}
\hline $\operatorname{dim} H$ & $\begin{array}{l}\text { Nontrivial } \\
\text { examples }\end{array}$ & Classification & Remarks \\
\hline$p$ & - & 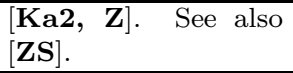 & $H \simeq k \mathbb{Z}_{p}$ \\
\hline$p^{2}$ & - & [M7]. & $\begin{array}{l}H \simeq k G, G \text { abelian of order } \\
p^{2}\end{array}$ \\
\hline$p q$ & - & {$[\mathrm{M} 4, \mathbf{E G}, \mathbf{G W}]$} & $\begin{array}{l}H \simeq k G, \text { or } k^{G} \text {. Other proofs } \\
\text { in }[\mathbf{S o}, \mathbf{N}] .\end{array}$ \\
\hline$p^{3}$ & $\begin{array}{l}\mathbf{K P}] \text { for } \operatorname{dim} 8 . \\
{[\mathbf{M} 7] \text { for } \operatorname{dim} 27 .}\end{array}$ & [M4, M7]. & $\begin{array}{l}\text { All semisolvable by } \\
{[\mathbf{M} 6, \mathbf{M W}]}\end{array}$ \\
\hline$p q^{2}$ & {$[\mathbf{F}, \mathbf{G}, \mathbf{M} 3, \mathbf{N}]$} & $\begin{array}{l}{[\mathbf{F}] \text { for } \operatorname{dim} 12,} \\
{[\mathbf{M} 3] \text { for } \operatorname{dim} 18,} \\
{[\mathbf{N}, \mathbf{N 2}, \mathbf{N 3}]}\end{array}$ & $\begin{array}{l}\text { All semisolvable. } \\
{[\mathbf{I K}, \text { Chapter X] for Kac al- }} \\
\text { gebras. }\end{array}$ \\
\hline$p^{4}(16)$ & {$[\mathbf{K}]$.} & {$[\mathbf{K}]$} & $\begin{array}{l}\text { All semisolvable by } \\
{[\mathbf{M} 6, \mathbf{M W}] .}\end{array}$ \\
\hline $\begin{array}{l}p^{3} q(24,40 \\
54,56)\end{array}$ & [IK] for $\operatorname{dim} 24$ & $\begin{array}{l}{[\mathbf{I K}, \text { Chapter XIV] }} \\
\text { for Kac algebras of } \\
\operatorname{dim} 24 .\end{array}$ & $\begin{array}{l}\text { Semisolvable; } \\
\text { Chapters } 6,9,12,13 .\end{array}$ \\
\hline$p q r(30,42)$ & $\begin{array}{l}\mathbf{A N}] \text { for } \operatorname{dim} 42 . \\
\text { nontrivial example in } \\
\operatorname{dim} 30 .\end{array}$ & Chapters $7,10$. & $\begin{array}{l}\text { Abelian extensions in } \\
\operatorname{dim} p q r \text { classified in }[\mathbf{N}] \text {. } \\
{[\mathbf{I K}, \text { Chapter X] for Kac al- }} \\
\text { gebras. }\end{array}$ \\
\hline$\overline{p^{5}(32)}$ & Yes. & - & $\begin{array}{l}\text { All semisolvable by } \\
{[\mathbf{M} 6, \mathbf{M W}] .}\end{array}$ \\
\hline$p^{2} q^{2}(36)$ & $\begin{array}{l}{[\mathbf{E G 4}] ; D\left(\mathbb{S}_{3}\right) ;} \\
\left(k D_{3} \times D_{3}\right)_{\phi} \text { simple } \\
{[\mathbf{G N}] ; \text { more. }}\end{array}$ & - & $\begin{array}{l}\text { First non-semisolvable } \\
\text { example. Chapter } 8 .\end{array}$ \\
\hline$p^{4} q(48)$ & Yes. & - & Semisolvable; Chapter 11 \\
\hline$p^{2} q r(60)$ & $\begin{array}{l}\left(k \mathbb{A}_{5}\right)_{\phi}(\text { simple })[\mathbf{N k}] \\
\left(k D_{3} \times D_{5}\right)_{\phi}(\text { simple }) \\
{[\mathbf{G N}] .}\end{array}$ & $\overline{-}$ & - \\
\hline
\end{tabular}

TABLE 1. Semisimple Hopf algebras of dimension $\leq 60$

categories of representations of these three Hopf algebras are not equivalent as monoidal categories. The results in this appendix have been motivated by the paper [Mo3], where the Schur indicators for the three Hopf algebras are compared: this gives evidence that the representation theory of $H_{8}$ is in some sense closer to that of $k D_{4}$ than to that of $k Q$. Here, $H_{8}$ denotes the unique nontrivial 8-dimensional semisimple Hopf algebra over $k$ [KP, M4], while $D_{4}$ and $Q$ denote, respectively, the dihedral and quaternionic groups of order 8 .

On the other hand, note that $H_{8}$ is an extension of $k^{\mathbb{Z}_{2} \times \mathbb{Z}_{2}}$ by $k \mathbb{Z}_{2}$, such that the bicrossproduct group $\left(\mathbb{Z}_{2} \times \mathbb{Z}_{2}\right) \bowtie \mathbb{Z}_{2}$ associated to the matched pair of the extension is $D_{4}$. As a consequence of [N5, Theorem 1.3], we know that $D\left(H_{8}\right)$ is a cocycle twist of the DijkgraafPasquier-Roche quasi-Hopf algebra $D^{\omega}\left(D_{4}\right)$, where $\omega \in H^{3}\left(D_{4}, k^{\times}\right)$ is the 3-cocycle associated to the extension corresponding to $H_{8}$ via the Kac exact sequence $[\mathbf{K a}]$. We present more evidence of this facts 
involving the Drinfeld doubles. More precisely, we prove on the one hand that $D\left(H_{8}\right)$ has no quotient Hopf algebra isomorphic to $k Q$. We also show that $D\left(H_{8}\right)$ is a (central) extension of $k^{G}$ by $k G$, where $G=G\left(D\left(H_{8}\right)^{*}\right) \simeq \mathbb{Z}_{2} \times \mathbb{Z}_{2} \times \mathbb{Z}_{2}$. See Theorems A.1.1, A.1.2.

Acknowledgements. Most of the results in this paper have been announced in [N4]. They were also communicated in the conferences Hopf algebras in Noncommutative Geometry and Physics (Brussels, May 2002) and Colloquium on Homology Theories, Representations and Hopf Algebras (Luminy, June 2002).

The author is grateful to N. Andruskiewitsch, S. Montgomery, L. Vainerman and H.-J. Schneider for interesting discussions, comments and references. She also thanks Y. Kashina and Y. Sommerhäuser for helpful remarks on a previous version of this paper, and the referee for many valuable comments.

This research has been done during a postdoctoral stay at the Department of Mathematics of the École Normale Supérieure, Paris. The author is grateful to Marc Rosso for his kind hospitality. 


\section{Conventions and Notation.}

Throughout, $k$ will denote an algebraically closed field of characteristic zero. The symbols Hom, $\otimes$, etc., will mean $\operatorname{Hom}_{k}, \otimes_{k}$, etc. Our references for the theory of Hopf algebras are [Mo, Sc]. The notation for Hopf algebras is standard; for instance, the group of group-like elements in $H$ is denoted by $G(H)$. For an algebra $A$ (respectively, for a coalgebra $C$ ) the notation $\operatorname{Hom}_{A}$ (resp. Hom ${ }^{C}$ ) is used to indicate the Hom bifunctor in the category of (left) $A$-modules (resp. $C$-comodules).

A Hopf algebra $H$ is called semisimple (respectively, cosemisimple) if it is semisimple as an algebra (respectively, if it is cosemisimple as a coalgebra). Let $H$ be a finite-dimensional Hopf algebra over $k$. By a result of Larson and Radford, it is known that $H$ is semisimple if and only if $H$ is cosemisimple, if and only if $\mathcal{S}^{2}=\mathrm{id}$. See $[\mathbf{L R}, \mathbf{L R 2}]$. The character algebra of $H$, denoted $R(H)$, is the subalgebra of $H^{*}$ spanned by the irreducible characters of $H$; if $H$ is semisimple, $R(H)$ coincides with the subalgebra of cocommutative elements in $H^{*}$.

Suppose $H$ is finite dimensional. For a Hopf subalgebra $A \subseteq H$, the index of $A$ in $H$ is defined by $[H: A]:=\frac{\operatorname{dim} H}{\operatorname{dim} A}$; it is an integer by [NZ]. Suppose $q: H \rightarrow B$ is a surjective Hopf algebra map, and identify $B^{*}$ with a Hopf subalgebra of $H^{*}$ via $q^{*}$; by abuse of terminology, the index $\left[H^{*}: B^{*}\right]$ will be also called the index of $B$ in $H$. 


\section{CHAPTER 1}

\section{Semisimple Hopf Algebras}

Along this chapter, $H$ will be a semisimple (thus finite-dimensional) Hopf algebra over $k$.

\subsection{Algebra structure}

As an algebra, $H$ is isomorphic to a direct product of full matrix algebras

$$
H \simeq k^{(n)} \times \prod_{d_{i}>1} M_{d_{i}}(k)^{\left(n_{i}\right)},
$$

where $n=\left|G\left(H^{*}\right)\right|$. It follows from the Nichols-Zoeller Theorem [NZ], that $n$ divides both $\operatorname{dim} H$ and $n_{i} d_{i}^{2}$, for all $i$. Moreover, by $[\mathbf{N R}]$ if $d_{i}=2$ for some $i$, then the dimension of $H$ is even.

By $[\mathbf{Z S}]$, if $n=1$, then $\left\{d_{i}: d_{i}>1\right\}$ has at least three elements.

Suppose that $A \subseteq H$ is a Hopf subalgebra. Then $A$ is also semisimple. Assume that $A$ is commutative. Then it follows from the Frobenius Reciprocity that $d_{i} \leq[H: A]$, for all $i$. See [AN2, Corollary 3.9].

If $H$ is as in (1.1.1) as an algebra, we shall say that $H$ is of type $\left(1, n ; d_{1}, n_{1} ; \ldots ; d_{r}, n_{r}\right)$ as an algebra. In this case, the dimension of the character algebra of $H$ is $n+n_{1}+\cdots+n_{r}$.

If $H^{*}$ is of type $\left(1, n ; d_{1}, n_{1} ; \ldots\right)$ as an algebra, we shall say that $H$ is of type $\left(1, n ; d_{1}, n_{1} ; \ldots\right)$ as a coalgebra.

So that $H$ is of type $\left(1, n ; d_{1}, n_{1} ; \ldots ; d_{r}, n_{r}\right)$ as a (co-)algebra if and only if $H$ has $n$ non-isomorphic one-dimensional (co-)representations, $n_{1}$ non-isomorphic irreducible (co-)representations of degree $d_{1}$, etc. Sometimes, we shall use the notation $X_{d}$ or $X_{d}(H)$ to indicate the set of irreducible characters of $H$ of degree $d$.

ExAMPLE 1.1.2. The above arguments can be used to get the possible algebra structures for a given finite dimension. For instance, suppose that $B$ is a semisimple Hopf algebra of dimension 60 such that $G\left(B^{*}\right)=1$. Then, as an algebra, $B$ is of one of the following types:

$$
(1,1 ; 3,2 ; 4,1 ; 5,1), \quad(1,1 ; 2,4 ; 3,2 ; 5,1) \quad \text { or } \quad(1,1 ; 2,4 ; 3,3 ; 4,1) .
$$




\subsection{Irreducible characters}

Let $V$ be an $H$-module. The character of $V$ is the element $\chi=$ $\chi_{V} \in H^{*}$ defined by $\langle\chi, h\rangle=\operatorname{Tr}_{V}(h), h \in H$. The degree of $\chi$ is the integer $\operatorname{deg} \chi=\chi(1)=\operatorname{dim} V$. If $U$ is another $H$-module, we have

$$
\chi_{V \otimes U}=\chi_{V} \chi_{U}, \quad \chi_{V^{*}}=\mathcal{S}\left(\chi_{V}\right) .
$$

Thus the irreducible characters, i.e., the characters of the irreducible $H$-modules, span a subalgebra $R(H)$ of $H^{*}$, called the character algebra of $H$. The antipode induces an anti-algebra involution $*: R(H) \rightarrow$ $R(H), \chi \mapsto \chi^{*}:=\mathcal{S}(\chi)$. The degree defines an augmentation $R(H) \rightarrow$ $k$.

We first resume some of the basic properties of $R(H)$ that will be often used in the rest of this paper. Proofs of these facts can be found in $[\mathbf{N R}]$.

Let $\chi_{V}, \chi_{W} \in R(H)$ be the characters of the $H$-modules $V$ and $W$, respectively. The integer $m\left(\chi_{V}, \chi_{W}\right)=\operatorname{dim}_{H_{H}}(V, W)$ will be called multiplicity of $V$ in $W$. This extends to a bilinear form $m$ : $R(H) \times R(H) \rightarrow k$.

Let $\widehat{H}$ denote the set of irreducible characters of $H$. If $\chi \in R(H)$, then we may write $\chi=\sum_{\mu \in \widehat{H}} m(\mu, \chi) \mu$. Let $\chi, \psi$ and $\lambda$ be characters of $H$-modules; we have

$$
m(\chi, \psi \lambda)=m\left(\psi^{*}, \lambda \chi^{*}\right)=m\left(\psi, \chi \lambda^{*}\right) .
$$

Let $\chi$ be an irreducible character of $H$. Denote by $G[\chi]$ the subgroup of $G\left(H^{*}\right)$ consisting of all those elements $g$ such that $g \chi=\chi$. We have

$$
g \in G[\chi] \Longleftrightarrow m\left(g, \chi \chi^{*}\right)>0 \Longleftrightarrow m\left(g, \chi \chi^{*}\right)=1 .
$$

See [NR, Theorem 10]. In particular,

$$
\chi \chi^{*}=\sum_{g \in G[\chi]} g+\sum_{\mu \in \widehat{H}, \operatorname{deg} \mu>1} m\left(\mu, \chi \chi^{*}\right) \mu .
$$

Note that $G\left[\chi^{*}\right]=\left\{g \in G\left(H^{*}\right): \chi g=\chi\right\}$. Also, if $a \in G\left(H^{*}\right)$, we have

$$
G[\chi a]=G[\chi], \quad G[a \chi]=a G[\chi] a^{-1} .
$$

As a consequence of $[\mathbf{N Z}]$, we have that $|G[\chi]| /(\operatorname{deg} \chi)^{2}$. Moreover, it follows from the results in [M5, Section 2], that the order of any element $g \in G[\chi]$ (hence also the exponent of $G[\chi]$ ) divides $\operatorname{deg} \chi$.

The following lemma will be applied later; see Lemma 12.1.1. It serves here to illustrate the (mostly well-known) fact that not any algebra type can arise as the structure of a semisimple Hopf algebra. 
LEMMA 1.2.4. There is no semisimple Hopf algebra with algebra type $(1,2 ; 2,1 ; 4, m), m \geq 1$.

Proof. Suppose on the contrary that $H$ is a semisimple Hopf algebra with this algebra type. Let $G\left(H^{*}\right)=\{\epsilon, g\}$ and let $\chi$ be the unique irreducible character of degree 2 of $H$; so that we have $g \chi=\chi=\chi g$, $\chi^{*}=\chi$ and $\chi^{2}=\epsilon+g+\chi$. In particular, $m(\chi, \zeta \chi)=m\left(\zeta, \chi^{2}\right)=0$, for all irreducible character $\zeta$ of degree 4 . Then also $m(\chi, \chi \zeta)=$ $m\left(\chi, \zeta^{*} \chi\right)=0$, for all such $\zeta$.

Let $\operatorname{deg} \zeta=4$ and write $\zeta \zeta^{*}=\epsilon+g+n \chi+\lambda$, where $\lambda$ is a character of $H$ such that $m(\chi, \lambda)=0$. By taking degrees, we get that $n>0$ and $n$ is odd. Since $n=m(\zeta, \chi \zeta)$, it follows that $n=1$. Therefore, $\chi \zeta=\zeta+\psi$, where $\operatorname{deg} \psi=4, \psi \neq \zeta$. Since $m(\chi, \chi \zeta)=0$, then $\psi$ is irreducible.

Then $m\left(\epsilon, \chi \zeta \psi^{*}\right)=m\left(\epsilon, \psi \psi^{*}\right)=1$, and we have $m\left(\chi, \zeta \psi^{*}\right)=1$. Thus $\zeta \psi^{*}=\chi+\sum_{l} \psi_{l}$, where $\operatorname{deg} \psi_{l}=4$. Taking degrees we get a contradiction. This shows that this type is not possible.

Let $n \geq 1$. The group $G\left(H^{*}\right) \times G\left(H^{*}\right)$ acts on the set $X_{n}$ via

$$
(g, h) \cdot \chi=g \chi h^{-1}, \quad g, h \in G\left(H^{*}\right), \quad \chi \in X_{p}
$$

We have $G[(g, h) \cdot \chi]=g G[\chi] g^{-1}$, for all $g, h \in G\left(H^{*}\right)$, and for all $\chi$.

Using this action we can get some information on the structure of the group $G\left(H^{*}\right)$. The following proposition gives an example of this fact; see also the proof of Lemma 10.1.6.

Proposition 1.2.6. Let $p<q$ be prime numbers. Suppose that $G\left(H^{*}\right)$ is nonabelian of order $p q$. Assume in addition that $G[\chi] \neq 1$, for all $\chi \in X_{p}$. Then $q^{2}$ divides $\left|X_{p}\right|$.

Proof. We may assume that $X_{p} \neq \emptyset$. Let $T \subseteq G\left(H^{*}\right)$ be a subgroup of order $q$. Consider the action $(T \times T) \times X_{p} \rightarrow X_{p}$ obtained by restriction of the action (1.2.5). We shall show that the stabilizer $(T \times T)_{\chi}$ is trivial, for all $\chi \in X_{p}$, which will imply the proposition.

Let $g, h \in T, \chi \in X_{p}$, and suppose that $(g, h) \cdot \chi=g \chi h^{-1}=\chi$. Then $G[\chi]=G[(g, h) \cdot \chi]=g G[\chi] g^{-1}$. By assumption, $G[\chi] \neq 1$, hence $|G[\chi]|=p$, because of the assumption $\left|G\left(H^{*}\right)\right|=p q$. Then $g=1$; otherwise, $G[\chi]$ would be a normal subgroup of order $p$ in $G\left(H^{*}\right)$, implying that $G\left(H^{*}\right)$ is abelian against the assumptions.

Hence we have $(g, h) \cdot \chi=\chi h^{-1}=\chi$, and $h^{-1} \in G\left[\chi^{*}\right]$. Thus also $h=1$. This finishes the proof of the proposition. 


\subsection{Coinvariants of Hopf algebra maps}

Let $q: H \rightarrow B$ be a Hopf algebra map and consider the subspaces of coinvariants

$$
\begin{aligned}
& H^{\operatorname{co} q}=\{h \in H:(\mathrm{id} \otimes q) \Delta(h)=h \otimes 1\}, \quad \text { and } \\
& { }^{\operatorname{co} q} H=\{h \in H:(q \otimes \mathrm{id}) \Delta(h)=1 \otimes h\} .
\end{aligned}
$$

Then $H^{\operatorname{co} q}$ (respectively, ${ }^{\text {co } q} H$ ) is a left (respectively, right) coideal subalgebra of $H$. We shall also use the notation $H^{\operatorname{co} B}:=H^{\operatorname{co} q}$. By $[\mathrm{Sc2}]$,

$$
\operatorname{dim} H=\operatorname{dim} H^{\operatorname{co} q} \operatorname{dim} q(H)=\operatorname{dim}^{\operatorname{co} q} H \operatorname{dim} q(H) .
$$

The left coideal subalgebra $H^{\operatorname{co} q}$ is stable under the left adjoint action of $H$. Moreover $H^{\operatorname{co} q}={ }^{\operatorname{co} q} H$ if and only if $H^{\operatorname{co} q}$ is a (normal) Hopf subalgebra of $H$. If this is the case, we shall say that the map $q: H \rightarrow B$ is normal.

REMARK 1.3.2. The Hopf algebra $B^{*}$ acts on $H$ on the left and on the right by $f \rightarrow h=\left\langle f, q\left(h_{2}\right)\right\rangle h_{1}$, and $h<f=\left\langle f, q\left(h_{1}\right)\right\rangle h_{2}$, respectively. Suppose that $q$ is surjective. Then we have

$$
\begin{aligned}
& H^{\operatorname{co} q}=\left\{h \in H: f \rightarrow h=\epsilon(f) h, \forall f \in B^{*}\right\}, \quad \text { and } \\
& { }^{\operatorname{co} q} H=\left\{h \in H: h \leftarrow f=\epsilon(f) h, \forall f \in B^{*}\right\} .
\end{aligned}
$$

In particular, let $\eta \in G\left(H^{*}\right)$ and consider the Hopf algebra map $q: H \rightarrow k^{\langle\eta\rangle}$ obtained by transposing the inclusion $k\langle\eta\rangle \subseteq H^{*}$. Then

$$
H^{\operatorname{co} q}=\{h \in H: \eta \rightarrow h=h\}, \quad{ }^{\operatorname{co} q} H=\{h \in H: h \leftarrow \eta=h\},
$$

where $<$ and $\rightarrow$ are the regular actions of $H^{*}$ on $H$.

By $[\mathbf{N Z}]$, if $A$ is a Hopf subalgebra of $H$ such that $A \subseteq H^{\operatorname{co} q}$, then $H^{\operatorname{co} q}$ is free as an $A$-module, with respect to the action by left multiplication of $A$. In particular, $\operatorname{dim} A$ divides $\operatorname{dim} H^{\operatorname{co} q}$. The same holds true with ${ }^{\text {co } q} H$ instead of $H^{\text {co } q}$. Indeed, with this $A$-module structure and the coaction given by the comultiplication of $H, H^{\operatorname{co} q}$ is a left $(A, H)$ Hopf module. We note the following consequence of this fact:

Lemma 1.3.3. Suppose that $|G(H)|$ and $\left[H^{*}: G\left(H^{*}\right)\right]$ are relatively prime. Then the group $G(H)$ is abelian and isomorphic to a subgroup of $\widehat{G\left(H^{*}\right)}$.

Proof. There is a surjective Hopf algebra map $\pi: H \rightarrow k^{G\left(H^{*}\right)}$, and we have $\operatorname{dim} H^{\operatorname{co} \pi}=\left[H^{*}: G\left(H^{*}\right)\right]$. If $1 \neq g \in G(H)$, then $\pi(g) \neq 1$, since otherwise $g$ would belong to $H^{\text {co } \pi}$ implying that the 
order of $g$ divides $\operatorname{dim} H^{\text {co } \pi}$, contradicting the assumption. Therefore the restriction of $\pi$ to $G(H)$ is injective, and $G(H)$ is thus isomorphic to a subgroup of $\widehat{G\left(H^{*}\right)}=G\left(k^{G\left(H^{*}\right)}\right)$. This implies that $G(H)$ is abelian.

Lemma 1.3.4. Suppose that $A \subseteq H$ is a Hopf subalgebra. Then $A^{\left.\operatorname{co} q\right|_{A}}=A \cap H^{\operatorname{co} q}$. In particular, $\operatorname{dim} A=\operatorname{dim}\left(A \cap H^{\operatorname{co} q}\right) \operatorname{dim} q(A)$.

Proof. The first claim is evident. The second follows from (1.3.1).

\subsection{Yetter-Drinfeld modules}

Let ${ }_{H}^{H} \mathcal{Y} \mathcal{D}$ denote the category of (left-left) Yetter-Drinfeld modules over $H$. Objects of this category are vector spaces $V$ endowed with an

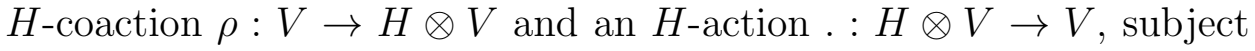
to the compatibility condition $\rho(h . v)=h_{1} v_{-1} \mathcal{S}\left(h_{3}\right) \otimes h_{2} . v_{0}, v \in V$, $h \in H$; morphisms are $H$-linear and colinear maps.

The category ${ }_{H}^{H} \mathcal{Y} \mathcal{D}$ is a modular category, which coincides as such with the category of modules over the Drinfeld double of $H$; see [EG]. It is shown in $[\mathbf{E G}]$ that if $V$ is a simple Yetter-Drinfeld module over $H$, then $\operatorname{dim} V$ divides $\operatorname{dim} H$.

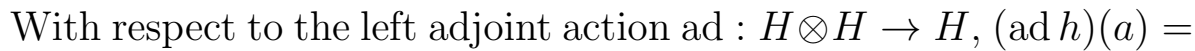
$h_{1} a \mathcal{S}\left(h_{2}\right)$ and the left regular coaction $\Delta: H \rightarrow H \otimes H, H$ becomes an object of ${ }_{H}^{H} \mathcal{Y} \mathcal{D}$.

The Yetter-Drinfeld submodules $V \subseteq H$ are exactly the left coideals $V$ of $H$ such that $h_{1} V \mathcal{S}\left(h_{2}\right) \subseteq V$, for all $h \in H$. Thus, a onedimensional Yetter-Drinfeld submodule of $H$ is exactly the span of a central group-like element of $H$.

It is well-kown that the space of (right) coinvariants of a Hopf algebra map is a left coideal stable under the left adjoint action. We thus obtain the following lemma:

Lemma 1.4.1. Let $H \rightarrow B$ be a Hopf algebra map. Then $H^{\operatorname{co} B}$ is a Yetter-Drinfeld submodule of $H$.

REMARK 1.4.2. Suppose that $H \rightarrow B \rightarrow B^{\prime}$ is a sequence of Hopf algebra maps. Then $H^{\text {co } B}$ is a Yetter-Drinfeld submodule of $H^{\text {co } B^{\prime}}$. In particular, since ${ }_{H}^{H} \mathcal{Y} \mathcal{D}$ is a semisimple category, there exists a YetterDrinfeld submodule $W \subseteq H^{\text {co } B^{\prime}}$ such that $H^{\text {co } B^{\prime}}=H^{\operatorname{co} B} \oplus W$.

We recover the following result, due to Kobayashi and Masuoka $[\mathbf{K M}]$; see also $[\mathbf{N}$, Theorem 2.1.1]. Our alternative proof is based on [EG]. 
Corollary 1.4.3. Suppose that $B \subseteq H$ is a Hopf subalgebra such that $[H: B]=p$ is the smallest prime number dividing $\operatorname{dim} H$. Then $B$ is a normal Hopf subalgebra and $H$ fits into a (co-)central extension $1 \rightarrow B \rightarrow H \rightarrow k \mathbb{Z}_{p} \rightarrow 0$.

Our argument proves indeed the following more precise statement: if $A \subseteq H$ is a normal Hopf subalgebra such that $\operatorname{dim} A$ is the smallest prime number dividing $\operatorname{dim} H$, then $A$ is central in $H$.

Proof. Consider the dual projection $H^{*} \rightarrow B^{*}$. So that we have $\operatorname{dim}\left(H^{*}\right)^{\text {co } B^{*}}=[H: B]=p$. Let $V$ be an irreducible Yetter-Drinfeld submodule of $\left(H^{*}\right)^{\text {co } B^{*}}$. Since the dimension of $V$ divides $\operatorname{dim} H$ and is less than $p$, we find that $\operatorname{dim} V=1$; therefore $V=k g$, for some $g \in Z\left(H^{*}\right) \cap G\left(H^{*}\right)$.

Decomposing $\left(H^{*}\right)^{\text {co } B^{*}}$ into irreducible Yetter-Drinfeld modules, we see that $\left(H^{*}\right)^{\text {co } B^{*}}$ is a central group-like Hopf subalgebra of $H^{*}$ of dimension $p$. This implies that $H^{*}$ fits into a central extension $0 \rightarrow k \mathbb{Z}_{p} \rightarrow H^{*} \rightarrow B^{*} \rightarrow 1$. The lemma follows after dualizing this extension.

\subsection{Yetter-Drinfeld modules and the character algebra}

In the paper $[\mathbf{Z 2}]$ Y. Zhu establishes a bijective correspondence between primitive idempotents in the character algebra $R(H) \subseteq H^{*}$ and irreducible Yetter-Drinfeld submodules of $H$. Indeed, it is shown in [Z2] that $D(H)$ and $R(H)$ form a commuting pair in End $H$, with respect to the $D(H)$-action corresponding to the Yetter-Drinfeld module structure in $H$ considered in Section 1.4 (or a version of it thereof) and the $R(H)$-action $\rightarrow: R(H) \otimes H \rightarrow H, f \rightarrow h:=\left\langle f, h_{2}\right\rangle h_{1}$.

Let $q: H \rightarrow B$ be a Hopf algebra projection. Then $H^{\operatorname{co} B}$ is a Yetter-Drinfeld submodule of $H$. Consider the dual inclusion of Hopf algebras $B^{*} \rightarrow H^{*}$ and let $e_{0} \in B^{*}$ be the normalized integral; $e_{0}$ is the primitive idempotent in $B^{*}$ corresponding to the trivial representation. Since $e_{0}$ is a cocommutative element, we have $e_{0} \in R(H)$. Hence we may write

$$
e_{0}=\Lambda+E_{1}+\cdots+E_{n}
$$

where $\Lambda, E_{1}, \ldots, E_{n}$ are orthogonal primitive idempotents in $R(H)$, such that $\Lambda$ is the normalized integral in $H^{*}$.

The following proposition gives a refinement of the result in $[\mathbf{Z 2}]$.

Proposition 1.5.2. The idempotents $\Lambda, E_{1}, \ldots, E_{n}$ in (1.5.1) correspond bijectively with the irreducible $H$-Yetter-Drinfeld submodules of $H^{\mathrm{co} B}$. 
Proof. We saw in Remark 1.3.2 that $H^{\text {co } B}$ coincides with the subalgebra of $B^{*}$-invariant elements of $H$ under the left regular action $\rightarrow: B^{*} \otimes H \rightarrow H$. Hence, since $e_{0} \in B^{*}$ is the primitive idempotent corresponding to the trivial representation, we have $H^{\text {co } B}=e_{0} \rightarrow H$. This implies the proposition.

\subsection{One dimensional Yetter-Drinfeld modules}

Let $g \in G(H), \eta \in G\left(H^{*}\right)$. Let $V_{g, \eta}$ denote the one dimensional vector space endowed with the action $h .1=\eta(h) 1, h \in H$, and the coaction $1 \mapsto g \otimes 1$. The following is a restatement of a result due to Radford [R, Proposition 10] that describes the group-like elements in the dual of $D(H)$.

Lemma 1.6.1. The one-dimensional Yetter-Drinfeld modules of $H$ are exactly of the form $V_{g, \eta}$, where $g \in G(H)$ and $\eta \in G\left(H^{*}\right)$ are such that $(\eta \longrightarrow h) g=g(h\llcorner\eta)$, for all $h \in H$.

Remark 1.6.2. Let $g \in G(H), \eta \in G\left(H^{*}\right)$. Then $V_{g, \eta}$ is a YetterDrinfeld module of $H$ if and only if $V_{\eta, g}$ is a Yetter-Drinfeld module of $H^{*}$.

Proof. We use Lemma 1.6.1. We have that $V_{g, \eta}$ is a YetterDrinfeld module of $H$ if and only if $(\eta \rightarrow h) g=g(h \leftarrow \eta)$, for all $h \in H$. This equivalent to $(g \rightarrow f) \eta=\eta(f<g)$, for all $f \in H^{*}$. Hence the claim follows.

LEMMA 1.6.3. Let $g \in G(H)$ and $\eta \in G\left(H^{*}\right)$ such that $V_{g, \eta}$ is a Yetter-Drinfeld module of $H$. Let also $q: H \rightarrow k^{\langle\eta\rangle}$ be the Hopf algebra map obtained by transposing the inclusion $k\langle\eta\rangle \subseteq H^{*}$.

Suppose that $V \subseteq H^{\mathrm{co} q}$ is a subspace of $H$ such that $g^{-1} v g=v$, for all $v \in V$. Then $V \subseteq{ }^{\operatorname{co} q} H$.

Proof. Using Lemma 1.6.1, we have for all $v \in V$,

$$
v<\eta=g^{-1}(\eta \rightarrow v) g=g^{-1} v g=v,
$$

the second equality because $v \in H^{\mathrm{co} q}$, see Remark 1.3.2. This shows that $v \in{ }^{\operatorname{co} q} H$ and finishes the proof of the lemma.

The following result will be used in Chapters 6 and 12 .

TheOREM 1.6.4. Let $g, \eta$ and $q$ be as in Lemma 1.6.3. Let $A \subseteq H$ be a Hopf subalgebra such that $g^{-1} a g=a$, for all $a \in A^{\operatorname{co} q}$. Then the restriction $\left.q\right|_{A}: A \rightarrow k^{\langle\eta\rangle}$ is normal.

Proof. We shall prove that $A^{\operatorname{co} q}$ is a Hopf subalgebra of $A$. By Lemma 1.6.3, we have $A^{\operatorname{co} q} \subseteq{ }^{\operatorname{co} q} A$. Thus ${ }^{\operatorname{co} q} A=A^{\operatorname{co} q}$, since they have the same finite dimension. This implies the theorem. 


\section{7. $H^{\text {co } B}$ as a left coideal of $H$}

Let $q: H \rightarrow B$ be a surjective Hopf algebra map. Identify $B^{*}$ with its image under the transpose map $q^{*}: B^{*} \rightarrow H^{*}$; so that $B^{*}$ is a Hopf subalgebra of $H^{*}$.

For each left $B^{*}$-module $W$, we may consider the induced left $H^{*}$ module $V=\operatorname{Ind}_{B^{*}}^{H^{*}} W:=H^{*} \otimes_{B^{*}} W$. Most basic properties of the induction functor are discussed, for instance, in [AN2]. Proposition 1.7.2 below establishes a relationship between the decomposition of the induced module $\operatorname{Ind}_{B^{*}}^{H^{*}} \epsilon$ and the normality of the map $q$. Here, $\operatorname{Ind}_{B^{*}}^{H^{*}} \epsilon$ indicates the representation induced from the trivial one-dimensional representation $W=k_{\epsilon}$ of $B^{*}$. The key ingredient is the following identification.

Recall that $H^{\operatorname{co} B}$ is a left coideal of $H$, hence a right $H^{*}$-module. Thus $\left(H^{\operatorname{co} B}\right)^{*}$ is naturally a left $H^{*}$-module through the action given by $\langle p . f, x\rangle:=\left\langle p, x_{1}\right\rangle\left\langle f, x_{2}\right\rangle$, for $p \in H^{*}, f \in\left(H^{\operatorname{co} B}\right)^{*}, x \in H^{\operatorname{co} B}$.

Lemma 1.7.1. $\left(H^{\text {co } B}\right)^{*} \simeq \operatorname{Ind}_{B^{*}}^{H^{*}} \epsilon$ as left $H^{*}$-modules.

As a consequence of this lemma, we see that for every irreducible left coideal $V$ of $H, V^{*}$ appears in $H^{\text {co } B}$ with the same multiplicity as $V$ does.

Proof. As left $H^{*}$-modules, $\operatorname{Ind}_{B^{*}}^{H^{*}} \epsilon=H^{*} \otimes_{B^{*}} k_{\epsilon} \simeq H^{*} / H^{*}\left(B^{*}\right)^{+}$. On the other hand, the evaluation map $\langle\rangle:, H^{*} \otimes H \rightarrow k$ induces a left $H^{*}$-linear isomorphism $H^{*} / H^{*}\left(B^{*}\right)^{+} \simeq\left(H^{\operatorname{co} B}\right)^{*}$.

Proposition 1.7.2. The map $q: H \rightarrow B$ is normal if and only if every irreducible $H^{*}$-module $V$ appears with multiplicity $\operatorname{dim} V$ or 0 in $\operatorname{Ind}_{B^{*}}^{H^{*}} \epsilon$.

Proof. We know that $q$ is normal if and only if $H^{\text {co } B}$ is a subcoalgebra of $H$. In turn, the last holds if and only if for every irreducible $H$-coideal $V \subseteq H^{\text {co } B}, H^{\text {co } B}$ contains the simple subcoalgebra corresponding to $V$; that is, if and only if, every irreducible left coideal of $H$ appears with multiplicity $\operatorname{dim} V$ or 0 in $H^{\operatorname{co} B}$. The proposition follows from Lemma 1.7.1. 


\section{CHAPTER 2}

\section{The Nichols-Richmond Theorem}

Recall from 1.2 that the character algebra of $H$, denoted $R(H)$, is the subalgebra of $H^{*}$ spanned by the irreducible characters of $H$.

A subalgebra $M$ of $R(H)$ is called a standard subalgebra if $M$ is spanned by irreducible characters of $H$. So that if $X$ is a subset of $\widehat{H}$, $X$ spans a standard subalgebra of $R(H)$ if and only if the product of characters in $X$ decompose as a sum of characters in $X$.

By [NR, Theorem 6] there is a bijection between standard subalgebras of $R(H)$ and quotient Hopf algebras of $H$. Under this bijection, the quotient $H \rightarrow B$ corresponds to the character algebra of $B$ : $R(B) \subseteq R(H)$.

\subsection{Irreducible characters of degree 2}

In this section we collect some facts related to the fusion rules of irreducible characters of degree 2.

Suppose that $H$ contains an irreducible character $\chi$ of degree 2, such that

$$
\chi^{2}=\sum_{g \in G[\chi]} g
$$

in particular, $\chi^{*}=\chi$ and $|G[\chi]|=4$. The set $G[\chi] \cup\{\chi\}$ spans a standard subalgebra of $R(H)$. This subalgebra corresponds to a quotient Hopf algebra $H \rightarrow \bar{H}$, where $\bar{H}$ is a semisimple non-commutative Hopf algebra of dimension 8 with algebra type $(1,4 ; 2,1)$. The classification of semisimple Hopf algebras of dimension 8 implies that the group $G[\chi]=G\left(\bar{H}^{*}\right)$ is not cyclic; a more general picture will appear later in 3.4 .

Conversely, every semisimple non-commutative Hopf algebra of dimension 8 has four linear characters, which constitute a group isomorphic to $\mathbb{Z}_{2} \times \mathbb{Z}_{2}$, and one (self-dual) irreducible character of degree 2 , satisfying the relations (2.1.1).

Suppose now that $H$ has an irreducible character $\chi$ of degree 2 such that

$$
\chi \chi^{*}=\epsilon+g+\chi,
$$


for some $g \in G\left(H^{*}\right)$. Then $G[\chi]=\{\epsilon, g\}, \chi^{*}=\chi$ and $G[\chi] \cup\{\chi\}$ spans a standard subalgebra of $R(H)$, which corresponds to a quotient Hopf algebra $H \rightarrow \bar{H}$, where $\bar{H} \simeq k \mathbb{S}_{3}$ is the unique non-commutative semisimple Hopf algebra of dimension 6 .

Proposition 2.1.3. Suppose that the following conditions are fulfilled:

(i) $|\{\chi \in \widehat{H}: \chi(1)=2\}|$ is odd;

(ii) $G\left(H^{*}\right)$ contains a subgroup $\Gamma$ of order 4 .

Then there is a quotient Hopf algebra $H \rightarrow \bar{H}$, where $\bar{H}$ is a semisimple non-commutative Hopf algebra of dimension 8 such that $\Gamma \subseteq \bar{H}^{*}$.

Proof. The group $\Gamma$ acts on the set $X_{2}=\{\chi \in \widehat{H}: \chi(1)=2\}$ in the form $g \cdot \chi=g \chi g^{-1}$. Let $X_{2}^{\prime} \subseteq X_{2}$ be the set of fixed points under this action. Since $\left|X_{2}\right|$ is odd by assumption, then $\left|X_{2}^{\prime}\right|$ is also odd. Moreover, since $\Gamma$ is abelian, $\Gamma$ acts on $X_{2}^{\prime}$ by left multiplication.

Let $Y \subseteq X_{2}^{\prime}$ be the set of fixed points of $X_{2}^{\prime}$ under left multiplication by $\Gamma$. Once again we find that $|Y|$ is odd and, in particular, that $Y$ is not empty. It is easy to see that $\mu^{*} \in Y$ for all $\mu \in Y$, whence there must exist $\chi \in Y$ such that $\chi^{*}=\chi$.

By construction, $\chi^{2}=\chi \chi^{*}=\sum_{g \in \Gamma} g$; see (1.2.3). Hence the set $\Gamma \cup\{\chi\}$ spans a standard subalgebra of $R(H)$ which corresponds to a Hopf algebra quotient of dimension 8 as claimed.

Lemma 2.1.4. Suppose that $\lambda \lambda^{*} \in k G\left(H^{*}\right)$ for some irreducible character $\lambda$. Assume in addition that $\operatorname{deg} \lambda \leq \operatorname{deg} \mu$ for all irreducible character $\mu$ with $\operatorname{deg} \mu>1$. Then also $\lambda^{*} \lambda \in k G\left(H^{*}\right)$.

ProOF. By assumption we have $\lambda \lambda^{*}=\sum_{g \in G[\lambda]} g$. On the other hand, we may write $\lambda^{*} \lambda=\sum_{g \in G\left[\lambda^{*}\right]} g+\sum_{\operatorname{deg} \mu>1} n_{\mu} \mu$, where $n_{\mu}=$ $m\left(\mu, \lambda^{*} \lambda\right)$. Then we have

$$
\lambda \lambda^{*} \lambda=\sum_{g \in G\left[\lambda^{*}\right]} \lambda g+\sum_{\operatorname{deg} \mu>1} n_{\mu} \lambda \mu=\left|G\left[\lambda^{*}\right]\right| \lambda+\sum_{\operatorname{deg} \mu>1} n_{\mu} \lambda \mu,
$$

and also

$$
\lambda \lambda^{*} \lambda=\sum_{g \in G[\lambda]} g \lambda=|G[\lambda]| \lambda .
$$

Comparing the multiplicity of $\lambda$ in both expressions, we find that $\lambda \mu=(\operatorname{deg} \mu) \lambda$, for all $\mu$ such that $n_{\mu} \neq 0$. Thus $\operatorname{deg} \mu=m(\lambda, \lambda \mu)=$ $m\left(\mu, \lambda^{*} \lambda\right)=n_{\mu}$. But then $n_{\mu} \operatorname{deg} \mu \geq(\operatorname{deg} \lambda)^{2}$ for all $\mu$ such that $\operatorname{deg} \mu>1$ and $n_{\mu} \neq 0$; so we see that $n_{\mu}=0$, for all $\mu$ such that $\operatorname{deg} \mu>1$. This finishes the proof of the lemma. 


\subsection{The Nichols-Richmond theorem}

The following theorem is due to Nichols and Richmond. See $[\mathbf{N R}$, Theorem 11]. We state here a version convenient to the finite dimensional context.

THEOREM 2.2.1. Suppose that $H$ has an irreducible character $\chi$ of degree 2. Then at least one of the following conditions holds:

(i) $G[\chi] \neq 1$;

(ii) $H$ has a Hopf algebra quotient of dimension 24, which has a degree one character $g$ of order 2 such that $g \chi \neq \chi$;

(iii) $H$ has a Hopf algebra quotient of dimension 12 or 60.

Notice that, as a consequence of the theorem, if $\operatorname{dim} H<60$ and $H$ has an irreducible character of degree 2 , then $G\left(H^{*}\right) \neq 1$.

REMARK 2.2.2. (i) Suppose that $H$ has an irreducible character $\chi$ of degree 2 such that $G[\chi]=1$. Then $H$ must also contain an irreducible character $\psi$ of degree 3 , which is necessarily self-dual, and such that $\chi \chi^{*}=\epsilon+\psi$.

Assume that $H$ has no irreducible character of degree 4 . Then $|G[\psi]|=3$ and $\psi^{2}=\sum_{g \in G[\psi]} g+2 \psi$; so that $G[\psi] \cup \psi$ spans a standard subalgebra of $R(H)$, which corresponds to a quotient Hopf algebra of type $(1,3 ; 3,1)$.

Proof. We follow the lines of the proof of Case 1 in $[\mathbf{N R}$, Theorem 11]. Since $H$ has no irreducible character of degree 4 and $m(\chi, \psi \chi)=1$, a counting argument implies that $\psi \chi$ is a sum of irreducible characters of degree 2. Moreover, it is easy to see that all these characters are conjugated to $\chi$. By [NR, Theorem $10(3)], \psi \chi=\sum_{g \in G[\psi]} g \chi$, so in particular $|G[\psi]|=3$. Multiplying both sides of this equation by $\chi^{*}$ on the right, we get $\psi^{2}=\sum_{g \in G[\psi]} g+2 \psi$. This proves the claim.

(ii) The assumption $G[\chi]=1$, for an irreducible character $\chi$ of degree $n$, implies also that $\chi$ has exactly $\left|G\left(H^{*}\right)\right|$ distinct conjugates under the action of $G\left(H^{*}\right)$ by left multiplication. Thus, in this case, we must have an inequality $\left|G\left(H^{*}\right)\right| \leq\left|X_{n}\right|$.

The following corollary of the Nichols-Richmond Theorem gives some restrictions for the possibility $G\left(H^{*}\right)=1$.

Corollary 2.2.3. Suppose that $H$ has an irreducible character of degree 2. If $G\left(H^{*}\right)=1$, then $H$ has a Hopf algebra quotient of dimension 60. In particular, 60/ $\operatorname{dim} H$. 
Proof. Any semisimple Hopf algebra of dimension 12 contains nontrivial group-like elements $[\mathbf{F}]$. By Theorem 2.2.1, if $G\left(H^{*}\right)=1, H$ must have a Hopf algebra quotient of dimension 60 as claimed.

\subsection{An application to $D(H)$}

In this section, we consider an application of Theorem 2.2.1 to the Drinfeld double of $H$.

Proposition 2.3.1. Suppose that $H$ is a semisimple Hopf algebra such that $\operatorname{dim} H<60$. If the Drinfeld double $D(H)$ has an irreducible character of degree 2 , then $G\left(D(H)^{*}\right) \neq 1$.

In particular, $D(H)$ is not simple; see [ $\mathbf{N}$, Corollary 2.3.2].

Proof. If $D(H)$ has an irreducible character of degree 2, then by Corollary 2.2.3, $G\left(D(H)^{*}\right) \neq 1$, unless there is a quotient Hopf algebra $q: D(H) \rightarrow B$, with $\operatorname{dim} B=60$ such that $G\left(B^{*}\right)=1$.

If this were the case, then by [NZ] $\operatorname{dim} H$ is divisible by 30 (since it must be divisible by 2, 3 and 5 ); thus by assumption, $\operatorname{dim} H=30$. The proposition follows from Theorem 2, which says that $H$ is necessarily trivial.

In our applications we shall combine the preceding proposition with the following fact.

LEMma 2.3.2. Suppose that $H$ has a Hopf subalgebra or quotient Hopf algebra of index 3. If $G\left(H^{*}\right) \cap Z\left(H^{*}\right)=1$ and $G(H) \cap Z(H)=$ 1 , then the Drinfeld double of $H$ contains an irreducible character of degree 2 .

Proof. We may assume that $H$ has a quotient Hopf algebra $H \rightarrow$ $B$ of index 3 ; the other case is dual, once we notice that $D\left(H^{* \text { cop }}\right) \simeq$ $D(H)^{\mathrm{op}} \simeq D(H)$. By assumption $\operatorname{dim} H^{\text {co } B}=3$, and by Lemma 1.4.1 $H^{\text {co } B}$ is a Yetter-Drinfeld submodule of $H$. Decomposing $H^{\operatorname{co} B}$ as a direct sum of irreducible Yetter-Drinfeld submodules implies the lemma, since the trivial appears with multiplicity 1.

\subsection{Existence of proper Hopf subalgebras}

In this section we apply the Nichols-Richmond theorem in order to assure, in certain cases, the existence of proper Hopf subalgebras.

LEMma 2.4.1. Let $\chi$ and $\psi$ be irreducible characters of $H$ such that the product $\chi \psi$ is irreducible. Then for all irreducible character $\mu \neq \epsilon$ we must have $m\left(\mu, \psi \psi^{*}\right)=0$ or $m\left(\mu, \chi^{*} \chi\right)=0$.

In particular $G[\psi] \cap G\left[\chi^{*}\right]=1$. 
Proof. Let $\zeta=\chi \psi$. By Schur's Lemma, $\zeta$ is irreducible if and only if $m\left(\epsilon, \zeta \zeta^{*}\right)=1$.

On the other hand, we have

$$
\begin{aligned}
\zeta \zeta^{*} & =\chi \psi \psi^{*} \chi^{*}=\chi\left(\sum_{\mu \in \widehat{H}} m\left(\mu, \psi \psi^{*}\right) \mu\right) \chi^{*} \\
& =\chi \chi^{*}+\sum_{\mu \neq \epsilon} m\left(\mu, \psi \psi^{*}\right) \chi \mu \chi^{*} .
\end{aligned}
$$

Therefore, $m\left(\epsilon, \zeta \zeta^{*}\right)=1$ if and only if for all $\mu \neq \epsilon$, with $m\left(\mu, \psi \psi^{*}\right)>0$, we have $m\left(\epsilon, \chi \mu \chi^{*}\right)=0$ or equivalently, $m\left(\mu, \chi^{*} \chi\right)=0$. This proves the lemma.

TheOREM 2.4.2. Suppose that $1 \neq G[\chi] \cap G[\psi]$, for all irreducible characters $\chi$ and $\psi$ of degree 2 . Then there is a quotient Hopf algebra $\pi: H \rightarrow \bar{H}$, such that $\bar{H}$ is of type $\left(1,\left|G\left(H^{*}\right)\right| ; 2,\left|X_{2}\right|\right)$ as an algebra.

Proof. It follows from Lemma 2.4.1 and the assumptions, that if $\chi$ and $\psi$ are irreducible characters of degree 2 , then their product $\chi \psi$ is not irreducible. Thus $\chi \psi$ decomposes as a sum of irreducible characters of degree at most 2 ; indeed, the one-dimensional characters appearing in $\chi \psi$ with positive multiplicity form a coset of the stabilizer $G[\chi]$ in $G\left(H^{*}\right)$ and thus there are 0,2 or 4 of them, so that the other irreducible summands must be of degree 2 . Therefore, the set $\{\chi \in \widehat{H}: \chi(1) \leq 2\}$ spans a standard subalgebra of the character algebra of $H$, implying the claim.

We next collect some useful variations of this theorem.

REMARK 2.4.3. (i) If $H$ has no irreducible character of degree 4 and the dimension of $H$ is not divisible by 12 , then the set $G\left(H^{*}\right) \cup X_{2}$ spans a standard subalgebra of $R(H)$. Therefore, $H$ has a quotient Hopf algebra of type $\left(1,\left|G\left(H^{*}\right)\right| ; 2,\left|X_{2}\right|\right)$.

Proof. In this case the product of two characters of degree 2 cannot be irreducible. By Theorem 2.2.1, since 12 does not divide $\operatorname{dim} H$, then $G[\chi] \neq 1$ for all $\chi \in X_{2}$, implying that for all $\psi \in X_{2}, \chi \psi$ decomposes as a sum of irreducible characters of degree $\leq 2$ (arguing as in the proof of Theorem 2.4.2).

(ii) Suppose that $G[\chi] \neq 1$ for all $\chi \in X_{2}$. Assume in addition that $G\left(H^{*}\right)$ has a unique subgroup $F$ of order 2. Then all irreducible characters of degree 2 are stable under multiplication by $F$.

In particular $F \subseteq G[\psi] \cap G\left[\chi^{*}\right]$, for all irreducible characters $\psi$ and $\chi$ of degree 2 . 
(iii) Suppose that the action by right multiplication of $G\left(H^{*}\right)$ on $X_{2}$ is transitive and $|G[\chi]| \neq 1$ for some irreducible character of degree 2 . Then the set $G(H) \cup X_{2}$ spans a standard subalgebra of $R\left(H^{*}\right)$ which corresponds to a quotient Hopf algebra $H \rightarrow B$, such that $B$ is of type $\left(1,\left|G\left(H^{*}\right)\right| ; 2,\left|X_{2}\right|\right)$ as an algebra.

Proof. The assumption implies that $|G[\chi]| \neq 1$, for all irreducible character $\chi$ with $\operatorname{deg} \chi=2$ : indeed, the action of $G\left(H^{*}\right)$ by right multiplication, which is transitive, preserves $G[\chi]$ and this group is not trivial at least for one $\chi$. Therefore $\chi \chi^{*}$ belongs to the span of $G\left(H^{*}\right)$ and $X_{2}$.

Since the action of $G\left(H^{*}\right)$ on the set $X_{2}$ by right multiplication is transitive, for all $\chi^{\prime} \in X_{2}$, there exists $h \in G\left(H^{*}\right)$ such that $\chi^{\prime}=\chi^{*} h$; then $\chi \chi^{\prime}=\chi \chi^{*} h$ belongs to the span of $G\left(H^{*}\right) \cup X_{2}$.

This shows that the set $G\left(H^{*}\right) \cup X_{2}$ spans a standard subalgebra of $R(H)$ and implies the claim.

\subsection{Hopf subalgebras of index 3}

Using character theory, we shall show that for some Hopf algebra inclusions $A \subseteq H$, with $[H: A]=3$, there is a quotient Hopf algebra $H \rightarrow \bar{H}$, such that the simple $\bar{H}$-modules have dimension 1 or 2 .

Observe that, by Corollary 1.4.3, if $H$ has a Hopf subalgebra of index 3 which is not normal, then the dimension of $H$ must be even; hence $6 / \operatorname{dim} H$. The following theorem and its corollary give more precise information.

TheOrem 2.5.1. Let $A \subseteq H$ be a Hopf subalgebra such that $[H$ : $A]=3$. Suppose that $A$ is not normal in $H$. Then there exist a Hopf algebra $\bar{H}$ of algebra type $\left(1,\left|G\left(H^{*}\right)\right| ; 2, \frac{\left|G\left(H^{*}\right)\right|}{2}\right)$ together with surjective Hopf algebra maps $H \rightarrow \bar{H} \rightarrow B$, where $B \simeq k \mathbb{S}_{3}$, and a surjective coalgebra map $B \rightarrow H / H A^{+}$such that the following diagram is commutative:

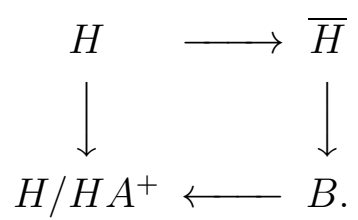

Proof. Since $A$ is not normal in $H$, by Proposition 1.7.2, $\operatorname{Ind}_{A}^{H} \epsilon=$ $\epsilon+\chi$, where $\chi$ is an irreducible character of degree 2. In particular, $\chi^{*}=\chi$, by Lemma 1.7.1.

Claim 2.5.2. $|G[\chi]|=2$ and $G[\chi] \cup\{\chi\}$ spans a standard subalgebra of $R(H)$. 
PROOF. It is enough to show that the product $\chi \chi^{*}=\chi^{2}$ admits a decomposition as in (2.1.2).

Since $m\left(\chi, \operatorname{Ind}_{A}^{H} \epsilon\right)=1$, we have $\left.\chi\right|_{A}=\epsilon+x$, where $\epsilon \neq x \in G\left(A^{*}\right)$, by Frobenius reciprocity. Hence $\left.\chi^{2}\right|_{A}=2(\epsilon+x)$.

In the character algebra of $H$ one of the following decompositions must hold:

(a) $\chi^{2}=\epsilon+a+b+c$, where $a, b, c \in G\left(H^{*}\right) \backslash\{\epsilon\}$ are pairwise distinct;

(b) $\chi^{2}=\epsilon+\psi$, where $\psi$ is an irreducible character of degree 3 ;

(c) $\chi^{2}=\epsilon+a+\lambda$, where $\epsilon \neq a \in G\left(H^{*}\right)$ and $\lambda$ is an irreducible character of degree 2 .

We shall show that the cases (a) and (b) are impossible, thus proving that $|G[\chi]|=2$. Suppose that (b) holds. Restricting to $A$, we find that necessarily $m\left(\epsilon,\left.\psi\right|_{A}\right)>0$. But by Frobenius reciprocity $m\left(\epsilon,\left.\psi\right|_{A}\right)=m\left(\psi, \operatorname{Ind}_{A}^{H} \epsilon\right)=0$; this contradiction discards case (b). Case (a) is similarly discarded.

Hence (c) holds. It remains to show that $\lambda=\chi$. For this, we restrict the equation $\chi^{2}=\epsilon+a+\lambda$ to $A$, and apply the Frobenius reciprocity to find that $m\left(\lambda, \operatorname{Ind}_{A}^{H} \epsilon\right)>0$, whence $\lambda=\chi$. This proves the claim.

Clearly, the standard subalgebra in Claim 2.5.2 corresponds to a quotient Hopf algebra $H \rightarrow B$, such that $B \simeq k \mathbb{S}_{3}$. Moreover, by construction, and using Lemma 1.7.1, $\left(H^{*}\right)^{\text {co } A^{*}} \subseteq B^{*} \subseteq H^{*}$. Hence there is a surjective coalgebra map $B \rightarrow H / H A^{+}$which factorizes the canonical map $H \rightarrow H / H A^{+}$.

Lemma 1.7.1 implies also that $g \chi g^{-1}=\chi$, for all $g \in G\left(H^{*}\right)$. Therefore, $G\left(H^{*}\right) \cup G\left(H^{*}\right) \chi$ spans a standard subalgebra of $R(H)$, which corresponds to a quotient Hopf algebra $H \rightarrow \bar{H}$ with the desired properties. This finishes the proof of the theorem.

Note that $\operatorname{dim} \bar{H}=3\left|G\left(H^{*}\right)\right|$. The following corollary is an immediate consequence of the theorem.

Corollary 2.5.3. Suppose that $A \subseteq H$ is a Hopf subalgebra such that $[H: A]=3$ and $A$ is not normal in $H$. Then we have

(i) $\left|G\left(H^{*}\right)\right|$ is even;

(ii) $3\left|G\left(H^{*}\right)\right|$ divides $\operatorname{dim} H$. 


\section{CHAPTER 3}

\section{Quotient Coalgebras}

Let $H$ be a semisimple Hopf algebra and let $A \subseteq H$ be a Hopf subalgebra. Consider the quotient coalgebra $p: H \rightarrow \bar{H}:=H / H^{+}$. By $[\mathbf{M} 2,3.4], \bar{H}$ is a cosemisimple coalgebra. In this chapter we aim to relate the corepresentations of $H$ and $\bar{H}$. We discuss the corepresentation theory of $\bar{H}$ in relation with that of $H$ and the corestriction functor ${ }^{H} \mathcal{M} \rightarrow{ }^{\bar{H}} \mathcal{M}$.

We show that if $C$ is a simple subcoalgebra of $H$ such that $C a \subseteq C$, for all $a \in A$, then the dual of the quotient coalgebra $C / C A^{+}$and the crossed product $A_{\alpha}$, where $\alpha: A \otimes A \rightarrow k$ is a certain 2-cocycle, constitute a commuting pair in $C^{*}$. This is applied in combination with Masuoka's main result in [M5], in some instances of the proof of Theorem 1.

In particular, when $A=k G$ is the group algebra of a subgroup $G$ of $G(H)$ and $V$ is a simple $H$-comodule, we deduce that $\operatorname{End}^{\bar{H}}(V)$ is isomorphic as an algebra to a twisted group algebra $k_{\alpha} \Gamma$, where $\Gamma \subseteq G$ is the stabilizer of $V$, i.e. $\Gamma=\{g \in G: V \otimes g \simeq V\}$, and $\alpha: \Gamma \times \Gamma \rightarrow k^{\times}$ is a 2-cocycle. This result implies that the multiplicity of an irreducible $\bar{H}$-comodule in $V$ is a divisor of the order of $\Gamma$. In particular, when the group $\Gamma$ is abelian, all irreducible $\bar{H}$-comodules in the restriction of $V$ to $\bar{H}$ appear with the same multiplicity $d$, where $d$ divides the order of $\Gamma$. This allows us to recover the result in [M5, Proposition 2.4].

Some of these results are applied to the case when $H$ is a biproduct in the sense of Radford: $H \simeq R \# A$. Indeed, in this case $R$ is isomorphic as a coalgebra to the quotient $H / H A^{+}$.

We shall denote by ${ }^{H} \mathcal{M}$ the category of left $H$-comodules; for a left $H$-comodule algebra $A$, the category of (left-right) $(A, H)$-Hopf modules will be indicated by ${ }^{H} \mathcal{M}_{A}$.

\subsection{A multiplicity formula}

Let $\rho: V \rightarrow H \otimes V, \rho(v)=v_{-1} \otimes v_{0}$, be a left $H$-comodule. Consider the $\bar{H}$-comodule structure on $V$ obtained by corestriction along the coalgebra map $p$; we shall sometimes use the notation $\bar{V}$ for 
this comodule structure, as well as $\bar{\rho}=(p \otimes \mathrm{id}) \rho: V \rightarrow \bar{H} \otimes V$ for the structure map. We obtain in this way a functor ${ }^{H} \mathcal{M} \rightarrow{ }^{\bar{H}} \mathcal{M}, V \mapsto \bar{V}$.

By a result of Schneider [Sc3, Theorem II], there is a category equivalence $\omega:{ }^{H} \mathcal{M}_{A} \rightarrow{ }^{\bar{H}} \mathcal{M}$ between the category of $(A, H)$-Hopf modules and the category of $\bar{H}$-comodules. The equivalence is given by $\omega: M \mapsto M / M A^{+}$, for any object $M$ of ${ }^{H} \mathcal{M}_{A}$.

Consider now the functor $F:{ }^{H} \mathcal{M} \rightarrow{ }^{H} \mathcal{M}_{A}, F(V)=V \otimes A$; where $H$ coacts diagonally on $V \otimes A$ and the right $A$-module structure is given by $v \otimes a . b=v \otimes a b$.

LEMMA 3.1.1. (i) The functor $F$ is a left adjoint of the forgetful functor $U:{ }^{H} \mathcal{M}_{A} \rightarrow{ }^{H} \mathcal{M}$;

(ii) for all left $H$-comodules $V$ there is an isomorphism $\omega F(V) \simeq \bar{V}$.

Proof. (i) We define natural maps

$$
\begin{gathered}
\psi: \operatorname{Hom}^{H}(X, U(Y)) \rightarrow \operatorname{Hom}_{A}^{H}(X \otimes A, Y), \\
\phi: \operatorname{Hom}_{A}^{H}(X \otimes A, Y) \rightarrow \operatorname{Hom}^{H}(X, U(Y)),
\end{gathered}
$$

as follows:

$$
\psi(f)(x \otimes a)=f(x) . a, \quad \phi(g)(x)=g(x \otimes 1),
$$

for all $x \in X, a \in A$. It is not hard to check that $\psi$ and $\phi$ are well defined and are indeed inverse isomorphisms.

(ii) The maps $\mu:(V \otimes A) /\left(V \otimes A^{+}\right) \rightarrow \bar{V}, \mu([v \otimes a]):=\epsilon(a) v$, and $\eta: \bar{V} \rightarrow(V \otimes A) /\left(V \otimes A^{+}\right), \eta(v):=[v \otimes 1]$, define inverse $\bar{H}$-colinear isomorphisms.

As a consequence we get the following proposition.

Proposition 3.1.2. Suppose that $U$ and $V$ are finite-dimensional left $H$-comodules. There is a natural linear isomorphism

$$
\operatorname{Hom}^{\bar{H}}(\bar{U}, \bar{V}) \simeq \operatorname{Hom}^{H}\left(V^{*} \otimes U, A\right) .
$$

Suppose that $V$ is a simple $H$-comodule. Then $\bar{V}$ is simple if and only if

$$
\operatorname{Hom}^{H}(V, V \otimes W)=\operatorname{Hom}^{H}\left(V^{*} \otimes V, W\right)=0,
$$

for all simple left $A$-comodule $k 1 \neq W$.

Proof. We have isomorphisms

$$
\begin{aligned}
\operatorname{Hom}^{H}(U, V \otimes A) & \simeq \operatorname{Hom}_{A}^{H}(U \otimes A, V \otimes A) \\
& \simeq \operatorname{Hom}^{\bar{H}}(\omega(U \otimes A), \omega(V \otimes A)) \simeq \operatorname{Hom}^{\bar{H}}(\bar{U}, \bar{V}) ;
\end{aligned}
$$


the first isomorphism by Lemma 3.1.1 (i), the second since $\omega$ is a category equivalence and the third by Lemma 3.1.1 (ii). This proves the first statement since $\operatorname{Hom}^{H}(U, V \otimes A) \simeq \operatorname{Hom}^{H}\left(V^{*} \otimes U, A\right)$.

As left $H$-comodule, $A$ decomposes in the form $A \simeq \oplus_{W}(\operatorname{dim} W) W$, where the sum runs over the set of isomorphism classes of irreducible left coideals of $A$, which coincides with the set of isomorphism classes of irreducible left coideals $W$ of $H$ which are contained in $A$. Hence we have

$$
\operatorname{Hom}^{\bar{H}}(\bar{V}, \bar{V}) \simeq \operatorname{Hom}^{H}\left(V^{*} \otimes V, A\right) \simeq \oplus_{W}(\operatorname{dim} W) \operatorname{Hom}^{H}\left(V^{*} \otimes V, W\right) ;
$$

this implies the last statement, in view of Schur's Lemma.

REMARK 3.1.3. (i) Suppose that $U$ and $V$ are finite-dimensional left $H$-comodules, and let $\chi_{U}$ and $\chi_{V} \in H$ be the corresponding characters. As a left $H$-comodule, $A \simeq \bigoplus_{\lambda \in \widehat{A^{*}}} \operatorname{deg} \lambda W_{\lambda}$. Proposition 3.1.2 implies the following multiplicity formula:

$$
\operatorname{dim} \operatorname{Hom}^{\bar{H}}(\bar{U}, \bar{V})=\sum_{\lambda \in \widehat{A}^{*}} \operatorname{deg} \lambda m\left(\lambda, \chi_{V}^{*} \chi_{U}\right) .
$$

(ii) Suppose that $A=k G$, where $G$ is a subgroup of $G(H)$. Let $G\left[V^{*}\right]$ denote the subgroup of $G$ consisting of all elements $g$ for which $V g \simeq V$; that is, $G\left[V^{*}\right]=G \cap G\left[\chi_{V}^{*}\right]$. Recall from (1.2.2) that for $g \in G$, we have $\operatorname{dim} \operatorname{Hom}^{H}\left(V^{*} \otimes V, g\right)=1$ if and only if $g \in G\left[V^{*}\right]$, and $\operatorname{dim} \operatorname{Hom}^{H}\left(V^{*} \otimes V, g\right)=0$ otherwise.

It follows from Proposition 3.1.2 that

$$
\operatorname{End}^{\bar{H}} V \simeq \operatorname{Hom}^{H}\left(V^{*} \otimes V, k G\right)=\bigoplus_{g \in G\left[V^{*}\right]} \operatorname{Hom}^{H}\left(V^{*} \otimes V, g\right) .
$$

We thus get $\operatorname{dim} \operatorname{End}^{\bar{H}} V=\left|G\left[V^{*}\right]\right|$.

\subsection{Stable subcoalgebras}

We keep the notation in the previous section. Let $C \subseteq H$ be a simple subcoalgebra and let $V \subseteq C$ be an irreducible left coideal of $H$. Suppose that $C a \subseteq C$, for all $a \in A$. That is, $C$ is a right $A$-module with action given by right multiplication; by $[\mathbf{N Z}] C$ is a free right $A$-module. Let $\bar{C}:=p(C)$ and let $t \in A$ be the normalized integral. We have $H=H(1-t) \oplus H t$, and $H A^{+}=H(1-t)$. In particular, $p(h)=p(h t)$, for all $h \in H$.

Notice also that End ${ }^{H} V=$ End $^{C} V$ and End ${ }^{\bar{H}} V=$ End $^{\bar{C}} V$.

LEMma 3.2.1. (i) The map $p: H \rightarrow \bar{H}$ induces an identification $C / C(1-t)=\bar{C}$;

(ii) $\operatorname{dim} \bar{C}=(\operatorname{dim} A)^{-1} \operatorname{dim} C$. 
Proof. (i) The map $p$ induces an identification $\bar{C}=C / C \cap H A^{+}=$ $C / C \cap H(1-t)$. We claim that $C \cap H(1-t)=C(1-t)$. Indeed, let $c \in C \cap H(1-t)$; then $c=h(1-t)$ implying, since $(1-t)$ is an idempotent, that $c=c(1-t) \in C(1-t)$. The other inclusion is immediate from the fact that $C a \subseteq C$, for all $a \in A$.

(ii) We have $C=C(1-t) \oplus C t$, since $t$ and $1-t$ are orthogonal idempotents, and $C$ is stable under right multiplication by $A$. By part (i) $\operatorname{dim} \bar{C}=\operatorname{dim} C t$. But $C t=C^{A}$ is the space of $A$-invariant elements in $C$ under the action by right multiplication. Since $C$ is a free right $A$ module of rank $(\operatorname{dim} A)^{-1} \operatorname{dim} C$, then $\operatorname{dim} C^{A}=(\operatorname{dim} A)^{-1} \operatorname{dim} C$.

REMARK 3.2.2. Let $\chi \in C$ be the irreducible character of $H$ corresponding to $C$. The simple subcoalgebra $C$ satisfies $C a \subseteq C$ for all $a \in A$ if and only if $\chi \psi \in \mathbb{Z} \chi$, for all $\psi \in \widehat{A^{*}}$. This follows from the fact that the multiplication map $m: H \otimes H \rightarrow H$ is a left (and right) $H$-comodule map.

Since $C$ is a right $A$-module coalgebra under right multiplication, then $C^{*}$ is a left $A$-module algebra under the action $(a . f)(c)=f(c a)$, $f \in C^{*}, c \in C, a \in A$.

By the Skolem-Noether Theorem for Hopf algebras [M9], since $C^{*}$ is a simple algebra over $k$, there exists a convolution invertible map $\psi: A \rightarrow C^{*}$ such that $a . f=\psi\left(a_{1}\right) f \psi^{-1}\left(a_{2}\right)$, for all $f \in C^{*}, a \in A$. This gives rise to an algebra map $\psi: A_{\alpha} \rightarrow C^{*}$, where $\alpha \in Z^{2}(A, k)$ is the 2-cocycle associated to $\psi$ in the form $\alpha(a, b)=\psi^{-1}\left(a_{1} b_{1}\right) \psi\left(a_{2}\right) \psi\left(b_{2}\right)$. Here, and elsewhere, $A_{\alpha}:=A \#_{\alpha} k$ denotes the associated crossed product with respect to the cocycle $\alpha$; that is, $A_{\alpha}=A$ as vector spaces, with the multiplication $a . b=\alpha\left(a_{2}, b_{2}\right) a_{1} b_{1}, a, b \in A$. Note that, when $A=k G$ is a group algebra, $(k G)_{\alpha}=k_{\alpha} G$ is the twisted group algebra.

Proposition 3.2.3. As algebras, $\left(\operatorname{End}^{\bar{C}} V\right)^{\text {op }} \simeq A_{\alpha}$. Moreover $A_{\alpha}$ and $\bar{C}^{*}$ form a commuting pair in $(\text { End } V)^{\mathrm{op}} \simeq C^{*}$.

Proof. The coalgebra projection $p: C \rightarrow \bar{C}$ induces by transposition an algebra inclusion $\bar{C}^{*} \subset C^{*}$. We have that $\bar{C}^{*}$ coincides with the subalgebra of invariants $\left(C^{*}\right)^{A}$ under the action of $A$. Indeed, $f \in \bar{C}^{*}$ if and only if $f(c)=f(p(c))$, for all $c \in C$, if and only if $f(c)=f(c t)=(t . f)(c)$, for all $c \in C$; whence, $\bar{C}^{*}=\left(C^{*}\right)^{A}$.

Hence, by definition of $\psi, \bar{C}^{*}$ coincides with the commutant of $\psi\left(A_{\alpha}\right)$ in $C^{*}$. Since $\bar{C}^{*}$ is semisimple, the double commutant theorem implies that $\psi\left(A_{\alpha}\right)$ is the commutant of $\bar{C}^{*}$ in $C^{*}$. 
It follows from Burnside's Density Theorem $[\mathbf{C R}]$ that there is an anti-isomorphism of algebras $I: C^{*} \rightarrow$ End $V$, given by

$$
I(f)(v):=\left\langle f, v_{-1}\right\rangle v_{0}, \quad v \in V, f \in C^{*} .
$$

Under this identification, the commutant of $\bar{C}^{*}$ in $C^{*}$ coincides with the subalgebra $\left(\operatorname{End}^{\bar{C}} V\right)^{\text {op }}$ of $(\text { End } V)^{\text {op }}$. Therefore $\psi$ defines a surjective algebra map $\psi: A_{\alpha} \rightarrow \psi\left(A_{\alpha}\right)=\left(\operatorname{End}^{\bar{C}} V\right)^{\text {op }}$.

Since $C A \subseteq C$, we have $\chi_{V} \lambda=(\operatorname{deg} \lambda) \chi_{V}$, for all irreducible character $\lambda \in \widehat{A^{*}}$; that is, $m\left(\lambda, \chi_{V}^{*} \chi_{V}\right)=\operatorname{deg} \lambda$, for all $\lambda \in \widehat{A^{*}}$. By Re$\operatorname{mark} 3.1 .3(\mathrm{i}), \operatorname{dim} \operatorname{End}^{\bar{C}} V=\sum_{\lambda \in \widehat{A^{*}}}(\operatorname{deg} \lambda)^{2}=\operatorname{dim} A$. Therefore $\psi: A_{\alpha} \rightarrow C^{*}$ is an injective algebra map and determines an isomorphism $A_{\alpha} \simeq\left(\text { End }^{\bar{C}} V\right)^{\mathrm{op}}$. This finishes the proof of the proposition.

COROLlaRY 3.2.5. There exists a bijective correspondence between irreducible $A_{\alpha}$-modules and irreducible $\bar{C}$-comodules.

There is an isomorphism $V \simeq \bigoplus_{i} U_{i} \otimes W_{i}$, where $W_{i}$ runs over $a$ system of representatives of the isomorphism classes of irreducible $A_{\alpha^{-}}$ modules and $U_{i}$ is the irreducible $\bar{C}$-comodule corresponding to $W_{i}$.

In particular, if $A=k G$ where $G$ is a finite group, then the multiplicity of $U_{i}$ in $V$ divides the order of $G$, for all $i$.

If $G$ is an abelian group, then all the irreducible $k_{\alpha} G$-modules have the same dimension $d$. Therefore all irreducible $\bar{C}$-subcomodules of $V$ appear with the same multiplicity $d$.

As an application of the methods of this section, we have the following proposition.

Proposition 3.2.6. Suppose that $A C=C=C A$. Assume in addition that $\operatorname{dim} A=\operatorname{dim} C$. Then $A$ is normal in $k[C]$.

Here, $k[C]$ denotes the subalgebra generated by $C$; this is a Hopf subalgebra of $H$ containing $A$.

Proof. By Lemma 3.2.1, $\operatorname{dim} C t=\operatorname{dim} t C=1$, where $t \in A$ is the normalized integral. Therefore $C t=t C=k \psi$, where $\psi \in C$ is the corresponding irreducible character. Hence, for all $c \in C, t c=l(c) \psi$ and $c t=r(c) \psi$, which implies that $t c=c t$, after applying the counit.

Hence $t$ commutes pointwise with $C$; thus it is central in $k[C]$, and a fortiori, $A$ is normal in $k[C]$.

REMARK 3.2.7. It follows from Corollary 3.2.5 that, if $G$ is cyclic and $|G|=\operatorname{dim} V$, then $\bar{C}$ is a cocommutative coalgebra. We thus recover a fact in the proof of [M5, Proposition 2.4]. 


\subsection{Quotients modulo group-like Hopf subalgebras}

Let $G$ be a subgroup of $G(H)$ and let $A=k G$. We shall now specialize the description in the previous sections.

Let $t:=\frac{1}{|G|} \sum_{g \in G} g$ be the normalized integral in $k G$.

Lemma 3.3.1. Let $C$ and $D$ be simple subcoalgebras of $H$. Then the following are equivalent:

(i) $p(C) \cap p(D) \neq 0$;

(ii) $p(C)=p(D)$;

(iii) There exists $g \in G$ such that $C g=D$.

Proof. (ii) $\Longrightarrow($ i). Clear, since $p(C) \neq 0$ for all simple subcoalgebra $C$.

(iii) $\Longrightarrow$ (ii). If $D=C g$, then $D t=C t$ and therefore $p(D)=$ $p(D t)=p(C t)=p(C)$.

(i) $\Longrightarrow$ (iii). Suppose that $c \in C$ and $d \in D$ are such that $p(c)=$ $p(d) \neq 0$. Then $c-d$ belongs to the kernel of $p$, and there exists $h \in H$ such that $c-d=h(1-t)$. Hence

$$
(c-d) t=h(1-t) t=0,
$$

implying that $c t=d t$. But $c t \in \sum_{g \in G} C g$ and $d t \in \sum_{g \in G} D g$. Therefore, $C g \cap D g^{\prime} \neq 0$, for some $g, g^{\prime} \in G$; since both $C g$ and $D g^{\prime}$ are simple subcoalgebras, this implies that $C g=D g^{\prime}$ and $D=C g\left(g^{\prime}\right)^{-1}$.

The group $G$ acts on the set of simple subcoalgebras of $H$ by right multiplication. Let $C_{1}, \ldots, C_{n}$ be a system of representatives of this action, and let $G_{i} \subseteq G$ be the stabilizer of $C_{i}$.

Corollary 3.3.2. There is an isomorphism of coalgebras $\bar{H} \simeq$ $\bigoplus_{i=1}^{n} \bar{C}_{i}$, where $\bar{C}_{i}=p\left(C_{i}\right) \simeq C_{i} / C_{i}\left(k G_{i}\right)^{+}$.

Proof. It follows from Lemma 3.3.1 that $\bar{H} \simeq \bigoplus_{i=1}^{n} p\left(C_{i}\right)$. Thus it remains to see that $p\left(C_{i}\right) \simeq C_{i} / C_{i}\left(k G_{i}\right)^{+}$.

Fix $1 \leq i \leq n$ and let $C=C_{i}, G_{C}=G_{i}$. We claim that $C \cap$ $H(k G)^{+}=C \cap C(1-t)=C \cap C\left(1-t_{C}\right)$, where $t_{C}=\frac{1}{\left|G_{C}\right|} \sum_{h \in G_{C}} h$ is the normalized integral in $k G_{C}$.

Note first that if $c \in C \cap H(k G)^{+}=C \cap H(1-t)$ then $c=h(1-t)$ and thus $c(1-t)=h(1-t)^{2}=c$; this shows that $C \cap H(k G)^{+}=C \cap C(1-t)$. On the other hand, we have

$$
t=\frac{1}{|G|} \sum_{g \in G} g=\frac{1}{|G|} \sum_{g \in G_{C} \backslash G} \sum_{h \in G_{C}} h g=\frac{\left|G_{C}\right|}{|G|} \sum_{g \in G_{C} \backslash G} t_{C} g .
$$


Thus, for $c \in C, c t=\frac{\left|G_{C}\right|}{|G|} \sum_{g \in G_{C} \backslash G} c t_{C} g$ belongs to $\bigoplus_{g \in G_{C} \backslash G} C g$, implying that $c t=0$ if and only if $c t_{C}=0$. Then $c \in C \cap C(1-t)$ if and only if $c t=0$ if and only if $c t_{C}=0$ if and only if $c \in C \cap C\left(1-t_{C}\right)=$ $C\left(1-t_{C}\right)$. This proves the claim and the corollary follows.

\subsection{On the structure of $G(H)$}

In this section we present some consequences of Proposition 3.2.3. Some of them are special cases of results in the papers $[\mathbf{T Y}, \mathbf{T}]$. We keep the notation in the previous sections: $C \subseteq H$ is a simple subcoalgebra, $V \subseteq C$ is an irreducible left coideal, $G$ is a subgroup of $G(H)$ such that $C g=C$, for all $g \in G$, and $\bar{C}=C / C(k G)^{+}$is the quotient coalgebra.

Proposition 3.4.1. Suppose that $\bar{C}$ is a simple coalgebra. Then there exists a non-degenerate 2 -cocycle $\alpha: G \times G \rightarrow k^{\times}$. In particular, the group $G$ is solvable and not cyclic.

Proof. By Corollary 3.2.5 the twisted group algebra $k_{\alpha} G$ is simple. This implies the proposition.

Corollary 3.4.2. Suppose that $|G|=\operatorname{dim} C=(\operatorname{dim} V)^{2}$. Then $G$ is solvable and not cyclic.

REMARK 3.4.3. Suppose as above that $|G|=\operatorname{dim} C$. If we assume in addition that $V^{*} \simeq V$, then $A=k G \oplus C$ is a Hopf subalgebra whose category $\mathcal{C}$ of corepresentations has necessarily the fusion rules in $[\mathbf{T Y}]$. The results in loc. cit. imply that $G$ is abelian. Since by definition $\mathcal{C}$ admits a fiber functor, the existence of a non-degenerate 2-cocycle on $G$ is a consequence of $[\mathbf{T}]$.

In the following proposition we give a Hopf theoretical proof of these facts concerning $G$, under rather less restrictive assumption.

Proposition 3.4.4. Suppose that the following conditions hold:

(i) $|G|=\operatorname{dim} C$;

(ii) $g C=C=C g$, for all $g \in G$.

Then the group $G$ is abelian and admits a non-degenerate 2 -cocycle.

If in addition $C=\mathcal{S}(C)$, then $A=k G \oplus C$ is a Hopf subalgebra of $H$ of dimension $2 \operatorname{dim} C$, which fits into a cocentral extension $1 \rightarrow$ $k^{\widehat{G}} \rightarrow A \rightarrow k \mathbb{Z}_{2} \rightarrow 0$.

An analogous result, in the context of Kac algebras, appears in [IK, Theorem IX.8 (ii)]. 
ProOF. Keep the notation in the proof of Proposition 3.2.3. Let $X_{g} \in C^{*}$ denote the image of $g \in G$ under the map $\psi: G \rightarrow C^{*}$; so that $\left\{X_{g}: g \in G\right\}$ is a basis of $C^{*}$ and $X_{a} X_{b}=\alpha(a, b) X_{a b}$, for all $a, b \in G$. Also, the action $(a . p)(c)=p(c a)$ is given by $a . p=X_{a} p X_{a}^{-1}$, for all $p \in C^{*}, a \in G$.

In view of condition (ii), the same arguments apply to the Hopf algebra $H^{\mathrm{op}}$. Therefore there exists a basis $\left\{Y_{g}: g \in G\right\}$ of $C^{*}$ such that the action $(p . b)(c)=p(b c)$ is given by $p . b=Y_{b}^{-1} p Y_{b}$, for all $p \in C^{*}$, $b \in G$. The relation $(a . p) . b=a \cdot(p . b)$ implies that $X_{a} Y_{b} X_{a}^{-1} Y_{b}^{-1}=$ $\zeta(a, b) 1$, for some map $\zeta: G \times G \rightarrow k^{\times}$. The definition of $\zeta$ implies that $a . Y_{b}=\zeta(a, b) Y_{b}$ and similarly that $X_{a}^{-1} \cdot b=\zeta(a, b) X_{a}^{-1}$. Thus, by the associativity of the actions, we get that $\zeta$ is a bicharacter on $G$.

Suppose that there exists $a \in G$ such that $\zeta(a, b)=1$, for all $b \in G$. This implies that $a . Y_{b}=Y_{b}$, for all $b \in G$. Since $\left\{Y_{b}\right\}_{b \in G}$ is a basis of $C^{*}$, we get that the action of $a$ on $C^{*}$ (and thus on $C$ ) is trivial. This implies that $a=1$, because by $[\mathbf{N Z}] C^{*} \simeq k G$ as a left and right $k G$ module. Therefore the bicharacter $\zeta: G \times G \rightarrow k^{\times}$is non-degenerate. This proves that $G$ is abelian as claimed. Finally, since $k G$, which is isomorphic to $k^{\widehat{G}}$, has index 2 in $A$, the last part of the proposition follows; see Corollary 1.4.3.

\subsection{A criterion of normality}

We review in this section a result of A. Masuoka, which appears in [M5, Section 2].

We shall assume that $C \subseteq H$ is a simple subcoalgebra of dimension $n^{2}$ of $H$, and $g \in G(H)$ is a group-like element of order $n$ such that $g C=C=C g$. In particular, $g \in k[C]$, hence $k\langle g\rangle \subseteq k[C]$.

Let $V \subseteq C$ be an irreducible left coideal, so that $k g \otimes V \simeq V \simeq$ $V \otimes k g$. Let also $\alpha: k g \otimes V \rightarrow V$ and $\alpha^{\prime}: V \otimes k g \rightarrow V$ be $H$-colinear isomorphisms.

LEMMA 3.5.1. $k\langle g\rangle$ is a normal Hopf subalgebra of $k[C]$ if and only if $\alpha$ and $\alpha^{\prime}$ commute as endomorphisms of $V$.

Proof. Let $t \in k\langle g\rangle$ be the normalized integral. Then $k\langle g\rangle$ is normal in $k[C]$ if and only if $t c=c t$, for all $c \in C$. By [M5, Lemma $2.1]$ this is in turn equivalent to $\left[\alpha, \alpha^{\prime}\right]=0$ in End $V$.

Corollary 3.5.2. Suppose that $V$ is an irreducible left coideal of $C$ such that $g V=V=V g$. Then $k\langle g\rangle$ is normal in $k[C]$.

Proof. In this situation, we may take as $\alpha$ the left multiplication by $g$, and $\alpha^{\prime}$ the right multiplication by $g$. Since this endomorphisms commute with each other, the corollary follows from Lemma 3.5.1. 


\section{CHAPTER 4}

\section{Braided Hopf Algebras}

Let $A$ be a semisimple Hopf algebra and let ${ }_{A}^{A} \mathcal{Y} \mathcal{D}$ denote the braided category of Yetter-Drinfeld modules over $A$. Let $R$ be a semisimple braided Hopf algebra in ${ }_{A}^{A} \mathcal{Y} \mathcal{D}$. The results in this chapter concern the biproduct construction, as described in Section 4.1. This construction was introduced by Radford $[\mathbf{R 2}]$ and interpreted in categorical terms by Majid $[\mathbf{M j}, \mathbf{M j} \mathbf{2}]$.

\subsection{Radford-Majid biproduct construction}

Denote by $\rho: R \rightarrow A \otimes R, \rho(a)=a_{-1} \otimes a_{0}$, and.$: A \otimes R \rightarrow R$, the coaction and action of $A$ on $R$, respectively. So that the Yetter-Drinfeld compatibility condition reads as follows:

$$
\rho(h . a)=h_{1} a_{-1} \mathcal{S}\left(h_{3}\right) \otimes h_{2} . a_{0}, \quad \forall a \in R, h \in A .
$$

We shall use the notation $\Delta_{R}(a)=a^{1} \otimes a^{2}$ and $\mathcal{S}_{R}$ for the comultiplication and the antipode of $R$, respectively.

Thus our assumption amounts to the following conditions:

(4.1.2) $R$ is an $A$-module and $A$-comodule algebra;

(4.1.3) $R$ is an $A$-module and $A$-comodule coalgebra;

(4.1.4) $\Delta_{R}(a b)=a^{1}\left(\left(a^{2}\right)_{-1} \cdot b^{1}\right) \otimes\left(a^{2}\right)_{0} b^{2}$;

$$
\mathcal{S}_{R}\left(a^{1}\right) a^{2}=\epsilon_{R}(a) 1_{R}=a^{1} \mathcal{S}_{R}\left(a^{2}\right)
$$

Let $H=R \# A$ be the corresponding biproduct; so that $H$ is a semisimple Hopf algebra with multiplication, comultiplication and antipode given by

$$
\begin{gathered}
(a \# g)(b \# h)=a\left(g_{1} \cdot b\right) \# g_{2} h, \quad \Delta(a \# g)=a^{1} \#\left(a^{2}\right)_{-1} g_{1} \otimes\left(a^{2}\right)_{0} \# g_{2}, \\
\mathcal{S}(a \# g)=\left(1 \# \mathcal{S}\left(a_{-1} g\right)\right)\left(\mathcal{S}_{R}\left(a_{0}\right) \# 1\right),
\end{gathered}
$$

for all $g, h \in A, a, b \in R$; here we use the notation $a \# g$ to indicate the element $a \otimes g \in R \# A$. See $[\mathbf{R 2}]$.

Consider the natural maps $\pi: H \rightarrow A, \pi(r \# a)=\epsilon_{R}(r) a$, and $\iota: A \rightarrow H, \iota(a)=1 \otimes a$. Then $\pi$ is a Hopf algebra surjection and $\iota$ is 
a Hopf algebra injection. Moreover we have $\pi \iota=\mathrm{id}_{A}$ and

$$
R=H^{\mathrm{co} \pi}=\{h \in H:(\mathrm{id} \otimes \pi) \Delta(h)=h \otimes 1\},
$$

coincides, as an $A$-module and $A$-comodule algebra with the left coideal subalgebra of right $A$-coinvariants in $H$. On the other hand, the map id $\otimes \epsilon: H \rightarrow R$ induces an isomorphism of left $A$-module and $A$ comodule coalgebras

$$
R \simeq H / H A^{+}
$$

Indeed, the biproduct construction for finite dimensional Hopf algebras is characterized by these properties. Namely, suppose that there are Hopf algebra maps $\iota: A \rightarrow H$ and $\pi: H \rightarrow A$ such that $\pi \iota: A \rightarrow A$ is an isomorphism. Then the subalgebra $R:=H^{\text {co } \pi}$ of right coinvariants of $\pi$ has a natural structure of Yetter-Drinfeld Hopf algebra over $A$ such that the multiplication map $R \# A \rightarrow H$ induces a Hopf algebra isomorphism. This principle will be often used throughout this paper.

Typically, and mainly following the lines described in Chapter 2, we shall encounter a Hopf subalgebra $A \subseteq H$ and a surjective Hopf algebra map $\pi: H \rightarrow B$, where $H, A$ and $B$ are certain semisimple Hopf algebras such that $\operatorname{dim} A=\operatorname{dim} B$. After an analysis of the possible left coideal decompositions of $H^{\text {co } B}$, we shall be able sometimes to deduce that $A \cap H^{\mathrm{co} B}=k 1$ : this property guarantees the injectivity of $\left.\pi\right|_{A}$, and hence that $\left.\pi\right|_{A}: A \rightarrow B$ is an isomorphism. This will tell us that $H \simeq R \# A$ has the structure of a biproduct and will enable us to use the biproduct techniques that we discuss in the rest of this chapter.

One simple instance of this situation, frequently used along this paper, is described in the following lemma.

Lemma 4.1.9. Suppose $A \subseteq H$ is a cocommutative Hopf subalgebra and $\pi: H \rightarrow B$ is a surjective Hopf algebra map, such that $\operatorname{dim} A=$ $\operatorname{dim} B$ and $\operatorname{dim} A$ is relatively prime to $[H: A]$. Then $\left.\pi\right|_{A}: A \rightarrow B$ is an isomorphism, and $H \simeq R \# A$ is a biproduct.

Proof. It is enough to show that $A \cap H^{\mathrm{co} \pi}=k 1$. This follows from the assumptions, since $\operatorname{dim} A \cap H^{\operatorname{co} \pi}$ divides both $\operatorname{dim} A$ and $\operatorname{dim} H^{\operatorname{co} \pi}=[H: A]$.

\subsection{Coalgebra structure of $R$}

It turns out that $R$ is a normal left coideal subalgebra of $H$ as well as a quotient left $H$-module coalgebra through the identification $R \simeq H / H A^{+}$. Since $H$ is also cosemisimple, $R$ decomposes as a direct sum $R=\oplus_{i} V_{i}$, where $V_{i}$ are irreducible left coideals of $H$. It is clear 
that any left coideal $V$ of $H$ such that $V \subseteq R$ is an $A$-subcomodule of $R$.

The following lemma gives insight into the relationship between the $H$-comodule structure and the coalgebra structure on $R$.

Proposition 4.2.1. Let $V \subseteq R$ be a left coideal of $H$. Then the following hold:

(i) $V$ is a left coideal of $R$;

(ii) $\operatorname{dim} \operatorname{End}^{R}(V) \leq \operatorname{dim} V$, and the equality holds if and only if $V$ is a subcoalgebra of $R$. If this is the case, and if $V$ is an irreducible left coideal of $H$, then $V$ has multiplicity 1 as a left $H$-subcomodule of $R$.

Proof. (i) The map $p=\mathrm{id} \otimes \epsilon: H \rightarrow R$ is a coalgebra surjection, and $\left.p\right|_{R}=\mathrm{id}_{R}$. Since $\Delta(V) \subseteq H \otimes V$, then $\Delta_{R}(V)=(p \otimes p) \Delta(V) \subseteq$ $R \otimes V$, showing that $V$ is a left coideal of $R$.

(ii) Let $V=\oplus_{i} m_{i} V_{i}$, where $V_{i}$ are simple, pairwise non-isomorphic, left coideals of $R$. Thus $m_{i} \leq \operatorname{dim} V_{i}$, and $\operatorname{dim} \operatorname{End}^{R}(V)=\sum_{i} m_{i}^{2} \leq$ $\operatorname{dim} V$. Moreover, $\operatorname{dim} \operatorname{End}^{R}(V)=\operatorname{dim} V$ if and only if $m_{i}=\operatorname{dim} V_{i}$, for all $i$, if and only if $V$ is a subcoalgebra of $R$.

Suppose now that $V$ and $U$ are irreducible coideals of $H$ such that $V$ is a subcoalgebra of $R$ and $V \simeq U \subseteq R$. Then $U \simeq V$ as left coideals of $R$. Since $V$ is a subcoalgebra of $R$, this implies that $U=V$. The proof of the proposition is now complete.

Corollary 4.2.2. Suppose that $V \subseteq R$ is a left coideal of $H$ and let $\chi$ be the character of $V$. Then we have

$$
\sum_{\lambda \in \widehat{A^{*}}} \operatorname{deg} \lambda m\left(\lambda, \chi_{V}^{*} \chi_{V}\right) \leq \operatorname{dim} V
$$

and the equality holds if and only if $V$ is a subcoalgebra of $R$.

In particular, $\left|G(A) \cap G\left[\chi^{*}\right]\right| \leq \operatorname{dim} V$.

Recall that $G\left[\chi^{*}\right]=\{g \in G(H): \chi g=\chi\}$.

Proof. Combine Proposition 4.2.1 (ii) with Remark 3.1.3.

REMARK 4.2.3. Suppose that $A=k G$ is a group algebra and $V \subseteq R$ is an irreducible left coideal of $H$ such that $\operatorname{dim} V=|G|$ and $V g \simeq V$, for all $g \in G$, as in $[\mathbf{N} 2,1.3]$.

Then we have $\operatorname{dim} \operatorname{End}^{R} V=|G|$ and, by Proposition 3.2.3, there is an isomorphism of algebras $\left(\operatorname{End}^{R} V\right)^{\text {op }} \simeq k_{\alpha} G$ for some $\alpha \in Z^{2}\left(G, k^{\times}\right)$.

On the other hand, by Proposition 4.2.1, $V$ is a subcoalgebra of $R$. So that End $^{R} V=\operatorname{End}^{V} V \simeq\left(V^{*}\right)^{\text {op }}$ as algebras. Whence $V$ is isomorphic to a dual twisted group algebra as a coalgebra. We thus recover the statement in [N2, Proposition 1.3.1]. 


\subsection{Hopf subalgebras}

In this section we discuss some results on the existence of proper Hopf subalgebras in the biproduct $H \simeq R \# A$.

LEMMA 4.3.1. Suppose that $\widetilde{R} \subseteq R$ is a subspace such that:

(1) $\widetilde{R}$ is a subalgebra and a subcoalgebra of $R$;

(2) there exists a Hopf subalgebra $B \subseteq A$ such that $\rho(\widetilde{R}) \subseteq B \otimes \widetilde{R}$ and $B . \widetilde{R} \subseteq \widetilde{R}$.

Then $\widetilde{\widetilde{R}}$ is a braided Hopf algebra over $B$ and the biproduct $\widetilde{R} \# B$ is a Hopf subalgebra of $H$.

Proof. It is not hard to see that the conditions (4.1.2), (4.1.3) and (4.1.4) are verified, so that $\widetilde{R}$ is a braided Hopf subalgebra of $R$. Using (4.1.6), we see that indeed $\widetilde{R} \# B$ is a Hopf subalgebra of $H$.

REMARK 4.3.2. (i) Suppose that $V \subseteq R$ is a subcoalgebra satisfying condition (2) in Lemma 4.3.1; that is, suppose that there exists a Hopf subalgebra $B \subseteq A$ such that $\rho(V) \subseteq B \otimes V$ and $B . V \subseteq V$.

Then the subalgebra $k[V]$ generated by $V$ in $R$ is both a subalgebra and a subcoalgebra, by (4.1.4); moreover $\rho(k[V]) \subseteq B \otimes k[V]$ and $B . k[V] \subseteq k[V]$ because the multiplication of $R$ is a comodule and module map. Therefore, Lemma 4.3.1 implies that $k[V]$ is a braided Hopf algebra over $B$ and $k[V] \# B$ is a Hopf subalgebra of $H$.

(ii) Suppose that there exists a Hopf subalgebra $B \subseteq A$ such that $\rho(R) \subseteq B \otimes R$. Then Lemma 4.3.1 applies with $\widetilde{R}=R$, and we find that $R \# B$ is a Hopf subalgebra of $H$.

LEMMA 4.3.3. Suppose that $V \subseteq R$ is an irreducible left coideal of $H$. Then we have

(i) $V$ is not irreducible as an $A$-subcomodule of $R$.

(ii) Assume in addition that $\operatorname{dim} V=2$ and $\sum_{g \in G(A)} g . V$ generates $R$ as an algebra. Then $\rho(R) \subseteq k G(A) \otimes R$ and $R \# k G(A)$ is a Hopf subalgebra of $H$.

Proof. (i) Let $\pi:=\epsilon \otimes$ id $: H \rightarrow A$ be the canonical Hopf algebra projection. We have $\rho(V) \subseteq A \otimes V$. Suppose on the contrary that $V$ is an irreducible $A$-subcomodule of $R$.

Then $\rho(V) \subseteq C_{0} \otimes V$, where $C_{0} \subseteq A$ is a simple subcoalgebra of dimension $(\operatorname{dim} V)^{2}$. Let $C \subseteq H$ be the simple subcoalgebra containing $V$. We have $\rho(V)=(\pi \otimes \mathrm{id}) \Delta(V) \subseteq \pi(C) \otimes V$, where $\pi: H \rightarrow A$ is the projection. Thus, $C_{0} \subseteq \pi(C)$, and since $\operatorname{dim} C=\operatorname{dim} C_{0}$, we find that $\left.\pi\right|_{C}$ is injective. This is absurd since $\left.\pi\right|_{V}=\left.\epsilon\right|_{V}$ because $V \subseteq R$.

(ii) By part (i), $V$ is not irreducible as an $A$-comodule and therefore $\rho(V) \subseteq k G(A) \otimes V$. By (4.1.1), $\rho(g . V) \subseteq k G(A) \otimes g . V$, for all $g \in G(A)$. 
This implies, in virtue of the assumption, that $\rho(R) \subseteq k G(A) \otimes R$. Thus, by Remark 4.3.2 (ii), $R$ is a braided Hopf algebra over $k G(A)$ and the biproduct $R \# k G(A)$ is a Hopf subalgebra of $H$.

Assume that $\operatorname{dim} A=p^{3}, p$ prime, and $A$ is not cocommutative. Then the index of $G(A)$ in $A$ is $p$ and $k G(A)$ in normal in $A$. Moreover, the irreducible $A$-comodules have dimension 1 or $p$. See $[\mathbf{M} 7]$. We thus obtain the following corollary.

Corollary 4.3.4. Let $H=R \# A$ be a biproduct, where $A$ is a non-cocommutative semisimple Hopf algebra of dimension $p^{3}$. Suppose that $V \subseteq R$ is an irreducible $p$-dimensional left coideal of $H$ such that $\sum_{g \in G(A)} g . V$ generates $R$ as an algebra.

Then $H$ contains a Hopf subalgebra of index $p$.

Proof. By Lemma 4.3.3 (i) $V$ is not irreducible as an $A$-comodule; therefore $\rho(V) \subseteq k G(A) \otimes V$. Then $\rho(R) \subseteq k G(A) \otimes R$, since by assumption the sum $\sum_{g \in G(A)} g . V$ generates $R$. Remark 4.3 .2 (ii) now implies that $R \# k G(A)$ is a Hopf subalgebra of $H$.

\subsection{Biproducts over finite groups}

Assume that $A=k G$ is the group algebra of a finite group $G$. Thus, $R$ is a $G$-graded algebra

$$
R=\bigoplus_{g \in G} R_{g}, \quad R_{g}=\{r \in R: \rho(a)=g \otimes a\},
$$

such that $\Delta_{R}\left(R_{g}\right) \subseteq \bigoplus_{s t=g} R_{s} \otimes R_{t}$. The action of $G$ is by algebra and coalgebra automorphisms, and we have

$$
h . R_{g}=R_{h g h^{-1}},
$$

for all $g, h \in G$. The braiding $\tau_{R, R}: R \otimes R \rightarrow R \otimes R$ is given by

$$
\tau_{R, R}(a \otimes b)=(g . b) \otimes a, \quad \forall a \in R_{g}, b \in R .
$$

Let $\operatorname{Supp} R \subseteq G$ denote the set of elements $g \in G$ such that $R_{g} \neq 0$. Let also $G_{R}$ denote the subgroup of $G$ generated by $\operatorname{Supp} R$.

LEMma 4.4.3. $G_{R}$ is a normal subgroup of $G$. Moreover, $R$ is a Yetter-Drinfeld Hopf algebra over $G_{R}$ with respect to the coaction $\rho$ and the restricted action of $G_{R}$ on $R$, and the biproduct $R \# k G_{R}$ is a normal Hopf subalgebra in $R \# k G$.

Proof. It follows from (4.4.1) that $G_{R}$ is normal in $G$. By Remark 4.3.2 (ii), $R \# k G_{R}$ is a Hopf subalgebra of $R \# k G$. It is normal thanks to (4.1.6). See [Mo2]. 
The following lemma will help to find, in certain cases, normal Hopf subalgebras of Hopf algebras obtained as biproducts.

LEMMA 4.4.4. (i) Suppose that $G$ contains a normal subgroup $N$ such that $N$ acts trivially on $R$. Then the group algebra $k N$ is a normal Hopf subalgebra in $H$.

(ii) Assume that $R$ is cocommutative and let $n=\operatorname{dim} R$. If $|G|$ does not divide $(n-1)$ ! then there exists a subgroup $1 \neq N$ of $G$ such that the group algebra $k N$ is normal in $H$.

Proof. (i) Since $N$ acts trivially on $R$, then $h a=a h$ in $H$, for all $h \in N, a \in R$. On the other hand, the Yetter-Drinfeld condition (4.4.1) implies that $N \subseteq Z_{G}\left(G_{R}\right)$.

Note the following consequence of (4.1.6):

$$
\operatorname{ad}_{a \# g}(b \# h)=\left(a^{1} \# a_{-2}^{2}\right)\left(g . b \# g h g^{-1} \mathcal{S}\left(a_{-1}^{2}\right)\right)\left(\mathcal{S}_{R}\left(a_{0}^{2}\right) \# 1\right) .
$$

In particular, for all $h \in N$, we have

$$
\begin{aligned}
\operatorname{ad}_{a \# g}(h) & =a^{1}\left(a_{-2}^{2}\left(g h g^{-1}\right) S\left(a_{-1}^{2}\right)\right) S_{R}\left(a_{0}^{2}\right) \\
& =a^{1}\left(g h g^{-1}\right) S_{R}\left(a^{2}\right)=\epsilon(a) g h g^{-1} \in k N ;
\end{aligned}
$$

the second equality because $g h g^{-1} \in N \subseteq Z_{G}\left(G_{R}\right)$. This proves (i).

(ii) Since $G$ acts on $R$ by coalgebra automorphisms fixing $1 \in G(R)$, then $G$ permutes the set $X=G(R) \backslash\{1\}$. This corresponds to a group homomorphism $f: G \rightarrow \mathcal{S}(X)$, where $\mathcal{S}(X)$ is the group of all permutations of $X$. Let $N$ be the kernel of $f$. By assumption $N \neq 1$ and $N$ acts trivially on $R$. Then the claim follows from part (i).

Proposition 4.4.6. Suppose that $\operatorname{dim} R=3$ or 4 . Then either $H$ or $H^{*}$ contains a proper normal cocommutative Hopf subalgebra.

ProOF. In this case, $R$ is necessarily cocommutative. By Lemma 4.4.4, we may assume that $|G|=2$ if $\operatorname{dim} R=3$ and $|G|=6,3$ or 2 if $\operatorname{dim} R=4$. In view of $[\mathbf{M} 4]$ and $[\mathbf{F}]$, it remains to consider the case where $|G|=6, \operatorname{dim} R=4$. In this case, $X=G(R) \backslash\{1\}$ has three elements and we may assume that $G \simeq \mathcal{S}(X)$ is not abelian; so that $\operatorname{dim} H=24$ and $|G(H)|$ is divisible by 6 . We may then also assume that $H$ is not trivial and $|G(H)| \neq 12$. Therefore $G=G(H)$ and $H$ is of type $(1,6 ; 3,2)$ as a coalgebra. The proposition will follow from the following claim.

Claim. The group $G$ is abelian.

Proof of the Claim. We have that $[H: G]=4$ and $\operatorname{dim} R\left(H^{*}\right)=8$. Consider the inclusion $k G \subseteq R\left(H^{*}\right)$. If $G$ is not abelian, then $R\left(H^{*}\right) \simeq$ $M_{2}(k) \times k^{(4)}$ as an algebra, and a complete set of orthogonal primitive 
idempotents of $k G$ is of the form $e_{0}, e_{1}, f_{0}, f_{1}$, where $\operatorname{dim} H e_{i}=4$ and $\operatorname{dim} H f_{j}=8$. Moreover, $f_{0}, f_{1}$ are not central.

The Kac-Zhu Theorem implies that $R\left(H^{*}\right)$ has a primitive idempotent $e$ with $\operatorname{dim} H e=2$, and by $[\mathbf{Z 2}] D(H)$ has an irreducible character of degree 2. By Proposition 2.3.1 $\left|G\left(D(H)^{*}\right)\right| \neq 1$. In view of $[\mathbf{R}]$, this implies that the subgroup of the group $G(D(H))=G\left(H^{*}\right) \times G(H)$ consisting of elements which are central in $D(H)$ is not trivial. We may assume that the elements of this group are of the form $\eta \otimes g$, where $g \neq 1$ if $\eta \neq \epsilon$. Thus $Z(G) \neq 1$ and the claim follows.

\subsection{Cocommutative braided Hopf algebras}

Suppose that $R$ is a cocommutative coalgebra, that is, $a^{1} \otimes a^{2}=$ $a^{2} \otimes a^{1}$, for all $a \in R$; so that all irreducible $R$-comodules are onedimensional.

Lemma 4.5.1. Let $V \subseteq H=R \# k G$ be an irreducible left coideal of dimension $\operatorname{dim} V>1$ and let $\chi=\chi_{V^{*}}$. Then $G[\chi] \cap G \neq 1$.

Proof. As coalgebras, $R \simeq H / H(k G)^{+}$. Let $C \subseteq H$ be the simple subcoalgebra containing $V$. Then the (right) stabilizer of $C$ in $G$ is $G[\chi] \cap G$. Consider the corestriction $\bar{V}$ of $V$ to $R$ as in Chapter 3 .

By Corollary 3.3.2, the image of $C$ under the canonical projection $H \rightarrow H / H(k G)^{+}$is isomorphic to $C / C(k(G[\chi] \cap G))^{+}$. Therefore, applying Proposition 3.2.3 with $A=k(G[\chi] \cap G)$, we find an isomorphism of algebras $\left(\text { End }^{R} \bar{V}\right)^{\text {op }} \simeq k_{\alpha}(G[\chi] \cap G)$, where $\alpha \in Z^{2}\left(G[\chi] \cap G, k^{\times}\right)$. Since $\operatorname{dim} V>1, \bar{V}$ cannot be irreducible. Therefore $|G[\chi] \cap G| \neq 1$ and the lemma follows.

Proposition 4.5.2. Let $|G|=q^{r}$, where $q$ is a prime number and $r>0$. Then $q$ divides the dimension of $V$, for all irreducible left coideals $V$ of $H$ such that $\operatorname{dim} V>1$.

Assume in addition that $R \cap G(H)=1$. Then $\operatorname{dim} R=1 \bmod q$.

Proof. Let $V$ be an irreducible left coideal of $H$ of dimension $\operatorname{dim} V>1$. It follows from Lemma 4.5.1 that $G\left[\chi_{V}\right] \cap G \neq 1$ and since $G\left[\chi_{V}\right] \cap G \subseteq G$, we find that $q$ divides $\left|G\left[\chi_{V}\right] \cap G\right|$.

Since $|G[V]|$ divides the dimension of $V$, it follows that $q / \operatorname{dim} V$.

Suppose now that $R \cap G(H)=1$. Observe that $R \cap G(H)$ coincides with the set $G(R)^{\text {co } \rho}$ of coinvariant group-like elements in $R$.

Since $R$ decomposes as a direct sum of irreducible left coideals of $H: R=k 1 \oplus V_{1} \oplus \cdots \oplus V_{m}$, and by the assumption $\operatorname{dim} V_{j}>1$, for all $j=1, \ldots, m$, the last claim follows. 


\subsection{Cocommutative braided Hopf algebras over $\mathbb{Z}_{p}$}

We begin this section by reviewing some of the results in [So], concerning the classification of cocommutative cosemisimple braided Hopf algebras over groups of prime order, that will be used later. We then show some applications.

The braided Hopf algebra $R$ is called trivial if the braiding $\tau_{R, R}$ : $R \otimes R \rightarrow R \otimes R$ is the canonical flip of vector spaces, i.e., $\tau_{R, R}(a \otimes b)=$ $b \otimes a$, for all $a, b \in R$. See [So, Definition 1.1].

By [Sb] $R$ is trivial if and only if $R$ is a (usual) Hopf algebra; that is, if and only if $\Delta_{R}(a b)=a^{1} b^{1} \otimes a^{2} b^{2}$, for all $a, b \in R$.

Let $p$ be a prime number and let $\mathbb{Z}_{p}$ denote the cyclic group of order $p$. Let $R$ be a braided semisimple Hopf algebra over $\mathbb{Z}_{p}$. Suppose that $R$ is a cocommutative coalgebra. It is shown in [So, Proposition 7.2] that if $R$ is nontrivial, then $p$ divides the dimension of $R$. More precisely, we have the following proposition.

Proposition 4.6.1. Let $H=R \# k \mathbb{Z}_{p}$ such that $H$ is not cocommutative. Suppose that $R$ is cocommutative. Then we have:

(i) Assume that $R$ is trivial. Then $H$ fits into an abelian central extension

$$
0 \rightarrow k \mathbb{Z}_{p} \rightarrow H \rightarrow R \rightarrow 1
$$

(ii) Assume that $R$ is not trivial. Then there are exact sequences of Hopf algebras

$$
\begin{aligned}
& 1 \rightarrow k^{\mathbb{Z}_{p}} \rightarrow H \rightarrow k F \otimes k \mathbb{Z}_{p} \rightarrow 1, \\
& 1 \rightarrow k^{\mathbb{Z}_{p}} \otimes k \mathbb{Z}_{p} \rightarrow H \rightarrow k F \rightarrow 1,
\end{aligned}
$$

for a certain group $F$.

Proof. (i) Since $R$ is cocommutative and trivial, it is isomorphic as a Hopf algebra to a group algebra $R \simeq k \Gamma$ and the action and coaction of $\mathbb{Z}_{p}$ on $R$ correspond, respectively, to actions by group automorphisms $\mathbb{Z}_{p} \rightarrow$ Aut $\Gamma$. By [So, Proposition 1.11], either the action or the coaction of $\mathbb{Z}_{p}$ on $R$ must be trivial. If the coaction is trivial, it follows from (4.1.6) that $H$ is cocommutative, against the assumption. Therefore the action is trivial, and again by (4.1.6), we find that the coalgebra surjection $\pi=$ id $\otimes \epsilon: H \rightarrow R$ is indeed a Hopf algebra surjection; moreover, we have $k \mathbb{Z}_{p}=H^{\mathrm{co} \pi}$. Therefore, $\pi$ gives rise to an abelian central extension (4.6.2).

(ii) By [So, Theorems 7.7 and 7.8], $R$ is isomorphic to a crossed product $k^{\mathbb{Z}_{p}} \#_{\sigma} k F$, where $F$ is a finite group, with 'diagonal' action and coaction of $k \mathbb{Z}_{p}$. These braided Hopf algebras fit into the construction 
described in Section 3 of [AN3]. Therefore, by [AN3, Proposition 3.7], there are exact sequences of Hopf algebras (4.6.3) and (4.6.4).

As an application, we shall give in the following theorem a classification result for certain semisimple Hopf algebras. A stronger result (without the assumption on $\left|G\left(H^{*}\right)\right|$ ) is proved in [IK, Corollary IX.9] in the context of Kac algebras.

THEOREM 4.6.5. Suppose that $H$ is of type $(1,2 ; 2, n)$ as a coalgebra. Assume in addition that $\left|G\left(H^{*}\right)\right|$ is even. Then $H$ is commutative.

Proof. We have $\operatorname{dim} H=2(2 n+1)$. In particular, since 4 does not divide $\operatorname{dim} H$, it follows from Theorem 2.2.1 that every irreducible character of degree 2 is stable under left multiplication by $G(H)$. Therefore, by Remark 3.2.7, $H / H(k G(H))^{+}$is a cocommutative coalgebra. By assumption, $G\left(H^{*}\right)$ contains a subgroup $\Gamma \simeq \mathbb{Z}_{2}$, and the projection $q: H \rightarrow k^{\Gamma}$ restricts injectively to $k G(H) \simeq k \mathbb{Z}_{2}$, because $\operatorname{dim} H^{\operatorname{co} q}=$ $2 n+1$ is odd. Hence $H:=R \# k \mathbb{Z}_{2}$, where $R \simeq H / H(k G(H))^{+}$is a cocommutative Yetter-Drinfeld Hopf algebra of odd dimension. By Proposition 4.6.1, since $H$ is not cocommutative, $H$ fits into an exact sequence $1 \rightarrow k \mathbb{Z}_{2} \rightarrow H \rightarrow R \rightarrow 1$, where $R$ is a cocommutative Hopf algebra. Hence $R=k F$, where $F$ is a group of order $2 n+1$.

Claim 4.6.6. The group $F$ is abelian.

Proof. By dualizing, we get an exact sequence $1 \rightarrow k^{F} \rightarrow H^{*} \rightarrow$ $k \mathbb{Z}_{2} \rightarrow 1$. Therefore, as an algebra, $H^{*}$ is isomorphic to a crossed product $H^{*} \simeq k^{F} \#_{\tau} k \mathbb{Z}_{2}$, corresponding to an action $\rightarrow: k \mathbb{Z}_{2} \times k^{F} \rightarrow k^{F}$ and a 2-cocycle $\tau: \mathbb{Z}_{2} \times \mathbb{Z}_{2} \rightarrow k^{F}$. By $[\mathbf{N}]$, we may assume that $\tau=1$.

The action is in this case of the form $g \rightarrow \delta_{x}=\delta_{g \triangleright x}$, for all $g \in \mathbb{Z}_{2}$, $x \in F$, where $\triangleright: \mathbb{Z}_{2} \times F \rightarrow F$ is an action by group automorphisms (because the extension is cocentral, see $[\mathbf{N}]$ ). By Clifford theory, the irreducible $H^{*}$-modules are exactly of the form $V_{x, U}=\operatorname{Ind}_{k^{F} \# k G_{x}}^{H^{*}} k x \otimes$ $U$, where $x \in F$ runs over a system of representatives of the orbits of $\mathbb{Z}_{2}$ in $F, G_{x}$ is the stabilizer of $x$ in $\mathbb{Z}_{2}$ and $U$ runs over all irreducible nonisomorphic $k G_{x}$-modules. Note that $\operatorname{dim} V_{x, U}=\left[\mathbb{Z}_{2}: G_{x}\right]$. The assumption on the coalgebra structure of $H$ implies that the action $\triangleright: \mathbb{Z}_{2} \times F \rightarrow F$ has exactly one fixed point $x=1$.

Let $\phi \in \operatorname{Aut} F, \phi(x)=g \triangleright x$, where $1 \neq g \in \mathbb{Z}_{2}$. Then $g$ is an automorphism of order 2 , with exactly one fixed point $x=1$. This implies that $F$ is abelian and moreover $g \triangleright x=x^{-1}$, for all $x \in F$.

The proof of the theorem can be now concluded as follows: we have a Hopf algebra inclusion $k^{F} \subseteq H^{*}$. Since the group $F$ is abelian, $k^{F}=k \widehat{F}$ is cocommutative, and thus $2 n+1$ divides the order of $G\left(H^{*}\right)$. 
Since by assumption the order of $G\left(H^{*}\right)$ is even, then $\left|G\left(H^{*}\right)\right|=\operatorname{dim} H$ and $H^{*}=k G\left(H^{*}\right)$ is cocommutative. One could alternatively use the more general statement on abelian exact sequences that we prove in Lemma 4.6.7 below.

LEMMA 4.6.7. Suppose that $H$ fits into an exact sequence

$$
1 \rightarrow k^{\mathbb{Z}_{2}} \rightarrow H \rightarrow k F \rightarrow 1
$$

where $F$ is an abelian group of odd order. Assume in addition that $H$ is of type $(1,2 ; 2, n)$ as a coalgebra. Then $H$ is commutative.

Proof. Note that the exact sequence is necessarily central, by the dual version of Corollary 1.4.3. Hence, in the associated matched pair $\left(\mathbb{Z}_{2}, F\right)$, the action $\triangleleft: \mathbb{Z}_{2} \times F \rightarrow \mathbb{Z}_{2}$ is trivial and the action $\triangleright: \mathbb{Z}_{2} \times F \rightarrow$ $F$ is by group automorphisms.

As an algebra, $H \simeq k^{\mathbb{Z}_{2}} \#_{\sigma} k F$ is a crossed product for the trivial action corresponding to $\triangleleft$ and for some 2-cocycle $\sigma: F \times F \rightarrow k^{\mathbb{Z}_{2}}$, and as a coalgebra $H \simeq k^{F} \#_{\tau} k \mathbb{Z}_{2}$ with respect to the action of $\mathbb{Z}_{2}$ on $k^{F}$ corresponding to $\triangleright$ and the trivial cocycle $\tau$; see $[\mathbf{N}]$.

To establish the lemma, it will be enough to show that the cocycle $\sigma$ is a coboundary, that is, it is symmetric.

Let $g$ be the generator of $\mathbb{Z}_{2}$. The assumption on the coalgebra structure of $H$ implies, as in the proof of Theorem 4.6.5, that $g$ acts on $F$ by $g \triangleright x=x^{-1}$ for all $x \in F$. Write

$$
\sigma(x, y)=\sigma_{1}(x, y) \delta_{1}+\sigma_{g}(x, y) \delta_{g}, \quad x, y \in F,
$$

where $\delta_{1}, \delta_{g} \in k^{\mathbb{Z}_{2}}$ are the canonical idempotents. The normalized cocycle condition for $\sigma$ implies that $\sigma_{g}: F \times F \rightarrow k^{\times}$is a 2-cocycle and $\sigma_{1}=1[\mathbf{M}]$. Moreover, the compatibility condition in [M, (4.8)] between $\sigma$ and the trivial cocycle $\tau$ implies that

$$
\sigma_{g}(x, y) \sigma_{g}\left(x^{-1}, y^{-1}\right)=1, \quad \forall x, y \in F .
$$

So that $\sigma_{g}\left(x^{-1}, y^{-1}\right)=\sigma_{g}(x, y)^{-1}$, for all $x, y \in F$. But the 2-cocycle $\beta: F \times F \rightarrow k^{\times}, \beta(x, y):=\sigma_{g}\left(x^{-1}, y^{-1}\right)$ is cohomologous to $\sigma_{g}$, because of the injectivity of the map

$\phi: H^{2}\left(F, k^{\times}\right) \rightarrow \operatorname{Hom}\left(\Lambda^{2}(F), k^{\times}\right), \quad \phi([\alpha])(x, y):=\alpha(x, y) \alpha(y, x)^{-1}$.

Hence, in $H^{2}\left(F, k^{\times}\right)$, we have $\left[\sigma_{g}\right]=[\beta]=\left[\sigma_{g}\right]^{-1}$, implying that $\left[\sigma_{g}\right]^{2}=1$, and a fortiori that $\left[\sigma_{g}\right]=1$ since the order of $F$ is odd. This finishes the proof of the lemma.

The following more general form of Lemma 4.6.7 was suggested by a comment of the referee. 
Corollary 4.6.8. Suppose that $H$ is of type $(1,2 ; 2, n)$ as a coalgebra. Assume in addition that $H$ fits into an exact sequence

$$
1 \rightarrow k^{\mathbb{Z}_{2}} \rightarrow H \rightarrow K \rightarrow 1,
$$

where $K$ is a Hopf algebra of odd dimension. Then $K$ is cocommutative and $H$ is commutative.

Proof. Note first that $K$ is cocommutative: this follows from Remark 3.2.7, since $K \simeq H / H(k G(H))^{+}$, and all simple subcoalgebras of dimension 4 are necessarily stable under left and right multiplication by $G(H)$. Thus $K \simeq k F$, for some group $F$ of odd order. In view of Lemma 4.6.7 the corollary will follow if we show that $K$ is also commutative. We do this as follows: consider the matched pair $\left(\mathbb{Z}_{2}, F\right)$ associated with $H$. As in the proof of Claim 4.6.6 we see that the assumption on the coalgebra structure of $H$ implies that the action $\triangleright: \mathbb{Z}_{2} \times F \rightarrow F$ is by group automorphisms and cannot have fixed points $x \neq 1$. Then the group is abelian, and $K$ is commutative.

\subsection{Transitive actions of central subgroups}

We shall assume in this section that $R$ contains a cocommutative subcoalgebra $V$ such that $\rho(V) \subseteq k G \otimes V$. As before, we shall use the notation $G_{V}$ to indicate the subgroup of $G$ generated by $\operatorname{Supp} V$. We have $\rho(V) \subseteq k G_{V} \otimes V$ and there is a basis $v_{1}, \ldots, v_{n}$ of $V$ such that $\rho\left(v_{i}\right)=g_{i} \otimes v_{i}$, where $g_{i} \in \operatorname{Supp} V, \forall i=1, \ldots, n$.

LEMMA 4.7.1. Suppose that there exists a central subgroup $S$ of $G$ with the property that $S$ permutes transitively the set $G(V)$. Then $G_{V}$ is an abelian subgroup of $G$ of order $\leq \operatorname{dim} V$.

Proof. Let $G(V)=\left\{a_{1}, \ldots, a_{n}\right\}$. Since $G(V)$ is a basis of $V$, we may write $\rho\left(a_{i}\right)=\sum_{j} t_{i j} \otimes a_{j}$, for some $t_{i j} \in k G$. As a consequence of the $k G$-colinearity of $\Delta_{R}$ we get the following relations:

$$
t_{i j} t_{i l}=\delta_{j l} t_{i j}, \quad \forall 1 \leq i, j, l \leq n .
$$

On the other hand, the coassociativity of $\rho$ implies that, for all $p=$ $1, \ldots, n$, the subspace $R_{p}$ spanned by the set $\left\{t_{p j}: 1 \leq j \leq n\right\}$ is a right coideal of $k G$; by definition, we have $k G_{V}=\sum_{p} R_{p}$.

The assumption on $S$ implies that $R_{p}=R_{j}$, for all $1 \leq p, j \leq n$. Fix $p$, and let $e_{j}:=t_{p j}, 1 \leq j \leq n$. By (4.7.2) we get that $k G_{V}$, which is spanned by $\left\{e_{j}\right\}$, is a commutative subalgebra of $k G$ of dimension $\leq n$. This proves the lemma. 


\section{CHAPTER 5}

\section{Cocycle Deformations of Some Hopf Algebras}

In this chapter, we describe some known examples of semisimple Hopf algebras as cocycle twists of group algebras. We also show that some others cannot be obtained in this fashion.

\subsection{Lifting from abelian groups}

Let $G$ be a finite group and let $\phi \in k G \otimes k G$ be a normalized invertible 2-cocycle. The following lemma is a consequence of [EG5].

LEMMA 5.1.1. The Hopf algebra $(k G)_{\phi}$ is cocommutative if and only if there exist a normal abelian subgroup $A \subseteq G$ and an ad $G$-invariant cohomology class $[\omega] \in H^{2}\left(\widehat{A}, k^{\times}\right)$such that

$$
\phi=\sum_{x, y \in \widehat{A}} \omega(x, y) \delta_{x} \otimes \delta_{y} \in A \otimes A,
$$

where, for $x \in \widehat{A}$, the element $\delta_{x} \in A$ is the primitive idempotent defined by $\delta_{x}:=\frac{1}{|A|} \sum_{a \in A} x(a) a$.

Proof. Note that $(k G)_{\phi}$ is cocommutative if and only if $\phi \phi_{21}^{-1}$ commutes with $\Delta(G)$. The 'if' direction follows from this observation and the invariance of $[\omega]$. The 'only if' part follows from [EG5, Proof of Theorem 1.3].

REMARK 5.1.3. Suppose that the 2-cocycle $\phi$ has the form (5.1.2). Then we say that $\phi$ is lifted from the (not necessarily normal) abelian subgroup $A$; see $[\mathbf{N k}]$.

Suppose that $\phi$ is lifted from the normal abelian subgroup $A$. Recall the isomorphism

$$
\theta: H^{2}\left(\widehat{A}, k^{\times}\right) \rightarrow \operatorname{Hom}\left(\Lambda^{2}(\widehat{A}), k^{\times}\right), \quad \theta([\sigma])(x, y)=\sigma(x, y) \sigma(y, x)^{-1} .
$$

This isomorphism commutes with the ad $G$-action (i.e., the action coming from the adjoint action of $G$ on $A$ ).

Then $(k G)_{\phi}$ is cocommutative if and only if $[\omega]$ is ad $G$-invariant in $H^{2}\left(\widehat{A}, k^{\times}\right)$.

In the rest of this chapter $p$ and $q$ will be distinct prime numbers. 
5.2. Examples in dimension $p q^{2} ; p=1 \bmod q$

Suppose that $p=1 \bmod q$. Let $\mathcal{A}_{i}, 0 \leq i \leq q-1$ be the nontrivial semisimple Hopf algebras constructed in $[\mathbf{G}]$. The Hopf algebras $\mathcal{A}_{i}$ are self-dual and we have $G\left(\mathcal{A}_{0}\right) \simeq \mathbb{Z}_{q} \times \mathbb{Z}_{q}$, while $G\left(\mathcal{A}_{i}\right) \simeq \mathbb{Z}_{q^{2}}$ for all $i=1, \ldots, q-1$; see $[\mathbf{A N}]$.

Let $F=\left\langle a: a^{p}=1\right\rangle$ be the cyclic group of order $p$ and let $\Gamma=\left\langle s, t: s^{q}=t^{q}=s t s^{-1} t^{-1}=1\right\rangle \simeq \mathbb{Z}_{q} \times \mathbb{Z}_{q}$.

Let $1<m, l \leq p-1$ be units modulo $p$ such that $m^{q}=l^{q}=1$ $\bmod p$. Consider the semidirect product $G=F \rtimes \Gamma$ corresponding to the action by group automorphisms of $\Gamma$ on $F$ defined on generators by $s . a=a^{m}$ and $t . a=a^{l}$.

Let $\omega \in Z^{2}\left(\widehat{\Gamma}, k^{\times}\right)$be a nontrivial 2-cocycle; in particular, the cohomology class $[\omega]$ generates $H^{2}\left(\widehat{\Gamma}, k^{\times}\right)=\operatorname{Hom}\left(\Lambda^{2} \widehat{\Gamma}, k^{\times}\right) \simeq \mathbb{Z}_{q}$. Consider the invertible normalized 2-cocycle $\phi=\sum_{x, y \in \Gamma} \omega(x, y) \delta_{x} \otimes \delta_{y} \in k \Gamma \otimes k \Gamma$.

The following proposition generalizes [Nk, Example 2.9]. It also follows from the results in [M5, Theorem 4.8] in the case where $p=3$ and $q=2$.

Proposition 5.2.1. (i) $\mathcal{A}_{0} \simeq(k G)_{\phi}$ as Hopf algebras;

(ii) Let $1 \leq i \leq q-1$. Then the Hopf algebras $\mathcal{A}_{i}$ cannot be obtained from a group algebra by twisting the comultiplication by means of a 2cocycle.

Proof. (i) We claim that the Hopf algebra $(k G)_{\phi}$ is nontrivial. Note that the twisted group algebra $k_{\omega} \Gamma$ is simple; so that $\phi$ is a minimal twist for $k \Gamma$ in the sense of [EG4]. Therefore we have

$$
k \Gamma=\left\{(f \otimes \mathrm{id})(b) ; f \in k^{\Gamma}\right\},
$$

where $b=\phi_{21}^{-1} \phi \in k \Gamma \otimes k \Gamma$.

Suppose that $(k G)_{\phi}$ is cocommutative. This is equivalent to the condition $b \Delta(g)=\Delta(g) b$, for all $g \in G$, or

$$
g b^{1} g^{-1} \otimes g b^{2} g^{-1}=b^{1} \otimes b^{2}, \quad \forall g \in G .
$$

Regard $\widehat{\Gamma}$ as a subgroup of $\widehat{G}$ by transposing the natural projection $G \rightarrow \Gamma$. For all $f \in \widehat{\Gamma}$, after applying $f \otimes$ id to both sides of the above equation, we have $g\left(f\left(b^{1}\right) b^{2}\right) g^{-1}=f\left(b^{1}\right) b^{2}$. Since $\widehat{\Gamma}$ spans $k^{\Gamma}$, this implies that $\Gamma$ is central in $G$, which is a contradiction. This establishes the claim.

Since $\Gamma$ is abelian and $\phi \in k \Gamma \otimes k \Gamma$, it follows $G\left((k G)_{\phi}\right)=\Gamma$. We know that $(k G)_{\phi}$ and $(k G)_{\phi}^{*}$ are of Frobenius type [EG3]. Therefore, by the classification results for semisimple Hopf algebras of dimension $p q^{2}\left[\mathbf{N 3}\right.$, Theorem 5.4.2], $(k G)_{\phi} \simeq \mathcal{A}_{0}$ as claimed. 
(ii) In this case we have $G\left(\mathcal{A}_{i}\right) \simeq \mathbb{Z}_{4}$. Suppose on the contrary that $\mathcal{A}_{i}=(k N)_{J}$, for some group $N$ and 2-cocycle $J \in k N \otimes k N$. By [EG4] there exists a subgroup $H$ of $N$ such that $J \in k H \otimes k H$ is a minimal twist for $k H$. In particular, $|H|$ is a square and therefore $|H|=q^{2}$. This forces $H \simeq \mathbb{Z}_{q} \times \mathbb{Z}_{q}$ in view of the minimality of $J$. But then $k H \subseteq$ $(k N)_{J}$ is a Hopf subalgebra of dimension $q^{2}$, necessarily isomorphic to $k G\left(\mathcal{A}_{i}\right)$. This is a contradiction. Thus part (ii) follows.

\subsection{Examples in dimension $p q^{2} ; q=1 \bmod p$}

Suppose that $q=1 \bmod p$. There is a family of Hopf algebras of dimension $p q^{2}$, constructed in $[\mathbf{N}]$ as a generalization of the examples in [M3]. This family is parametrized by the Hopf algebras $\mathcal{B}_{\lambda}, 0 \leq \lambda<$ $p-1$, where $\lambda \lambda^{\prime} \neq 1 \bmod p$.

We have $G\left(\mathcal{B}_{\lambda}\right) \simeq \mathbb{Z}_{q} \times \mathbb{Z}_{q}$ for all $\lambda,\left|G\left(\mathcal{B}_{\lambda}^{*}\right)\right|=p$ for $\lambda>0$, and $G\left(\mathcal{B}_{0}^{*}\right) \simeq \mathbb{Z}_{p q}$

Let $F$ and $\Gamma$ be as before. Let $G=G_{\lambda}$ be the semidirect product $\Gamma \rtimes F$ with respect to the action of $F$ on $\Gamma$ given by a.s $=s^{n}$, a.t $=t^{n^{\lambda}}$, where $0<n \leq q-1$ is such that $n^{p}=1 \bmod q$, and $0 \leq \lambda<p-1$.

Let $[\omega] \in H^{2}\left(\widehat{\Gamma}, k^{\times}\right)$be the 2-cocycle corresponding to the skewsymmetric non-degenerate bicharacter $\Omega: \widehat{\Gamma} \times \widehat{\Gamma} \rightarrow k^{\times}, \Omega(u, v)=$ $\operatorname{det}(u v)$.

We have $\Omega(a . u, a . v)=n^{\lambda+1} \Omega(u, v)$. Therefore, the bicharacter $\Omega$, and hence also the class $[\omega]$ is not ad $G$-invariant.

Consider the 2-cocycle $\phi \in k G \otimes k G$ lifted from $\Gamma$ as in Remark 5.1 .3 , corresponding to $\omega$.

Proposition 5.3.1. (i) $\mathcal{B}_{\lambda} \simeq\left(k G_{\lambda}\right)_{\phi}$ as Hopf algebras;

(ii) The Hopf algebras $\mathcal{B}_{\lambda}^{*}$ are not a cocycle deformation of any finite group.

In the case where $p=2$, part (i) of the proposition is contained in [M10, Proposition 3.2].

Proof. Part (i) follows from the above discussion, in view of the classification results for semisimple Hopf algebras of dimension $p q^{2}$ and the description in $[\mathbf{N}, 1.4]$.

Part (ii) follows from an argument similar to the one used to prove part (ii) of Proposition 5.2.1, since $\mathcal{B}_{\lambda}^{*}$ contains no Hopf subalgebra of square dimension.

\subsection{Normal Hopf subalgebras in cocycle twists}

Let $H$ be a semisimple Hopf algebra and let $\phi \in(H \otimes H)^{\times}$be a 2-cocycle. 
Lemma 5.4.1. Let $B \subseteq H$ be a Hopf subalgebra of $H$. Then $B$ is a Hopf subalgebra of $H_{\phi}$ if and only if $\phi(B \otimes B) \phi^{-1}=B \otimes B$. In this case, $B$ is normal in $H$ if and only if it is normal in $H_{\phi}$.

Note that the Hopf algebra structure on $B$ as a Hopf subalgebra of $H_{\phi}$ is not a priori that of a 2-cocycle twisting of $B$, since we may not have $\phi \in B \otimes B$.

Proof. The first part of the lemma follows easily. As for the second part, recall that $B$ is normal in $H$ if and only if $\mathrm{HB}^{+}=B^{+} H$, which depends only on the multiplication and the counit of $H$.

The following corollary is an easy consequence of the lemma.

Corollary 5.4.2. Suppose that $B \subseteq H$ is a central Hopf subalgebra. Then $B$ is a central Hopf subalgebra of $H_{\phi}$.

In particular, let $G$ be a finite group and let $\phi \in k G \otimes k G$ be a two cocycle. Then if $Z(G) \neq 1,(k G)_{\phi}$ contains a nontrivial central group-like element.

The remaining chapters will be devoted, respectively, to the proof of our main results on semisimple Hopf algebras of dimension 24, 30, $36,40,42,48,54$ and 56 . 


\section{CHAPTER 6}

\section{Dimension 24}

\subsection{Possible (co)-algebra structures}

Let $H$ be a nontrivial semisimple Hopf algebra of dimension 24 .

Lemma 6.1.1. The group $G\left(H^{*}\right)$ is of order $2,3,4,6,8$ or 12 . As an algebra, $H$ is of one of the following types:

- $(1,2 ; 2,1 ; 3,2)$,

- $(1,3 ; 2,3 ; 3,1)$,

- $(1,4 ; 2,5)$,

- $(1,4 ; 2,1 ; 4,1)$,

- $(1,6 ; 3,2)$,

- $(1,8 ; 2,4)$,

- $(1,8 ; 4,1)$,

- $(1,12 ; 2,3)$.

We shall prove later that the type $(1,4 ; 2,1 ; 4,1)$ is impossible, that is, there exists no semisimple Hopf algebra with this (co)algebra structure; see Lemma 6.1.7 below. The analogous result for Kac algebras follows from [IK, Proposition XIV.37], since there is no group $G$ for which $k G$ has this algebra structure.

Proof. The proof follows from counting arguments using 1.1.

REMARK 6.1.2. (i) If $H$ is of type $(1,12 ; 2,3)$ as an algebra, then $H$ is not simple by Corollary 1.4.3.

(ii) Suppose that $H$ is of type $(1,2 ; 2,1 ; 3,2)$ as an algebra. Then $H$ has a unique irreducible character $\chi$ of degree 2 , which necessarily satisfies (2.1.2) with $G\left(H^{*}\right)=\{\epsilon, g\}$. Therefore there is a quotient Hopf algebra $H \rightarrow k \mathbb{S}_{3}$, where $\mathbb{S}_{3}$ is the only non-abelian group of order 6 .

(iii) Suppose that $H$ is of type $(1,4 ; 2,5)$ or $(1,4 ; 2,1 ; 4,1)$ as a coalgebra. Then, by Proposition 2.1.3, $H$ contains a non-cocommutative Hopf subalgebra of dimension 8.

Lemma 6.1.3. Suppose that $H$ is of type $(1,3 ; 2,3 ; 3,1)$ as a coalgebra. Then $G\left(H^{*}\right) \cap Z\left(H^{*}\right) \neq 1$. 
Proof. It follows from Remark 2.2.2 (i) that $H$ has a Hopf subalgebra $K$ of dimension 12. Since the index of $K$ in $H$ is 2, then $G\left(H^{*}\right) \cap Z\left(H^{*}\right) \neq 1$.

Lemma 6.1.4. Assume that $H$ is of type $(1,6 ; 3,2)$ as a coalgebra. Then $H$ is not simple.

Proof. Suppose on the contrary that $H$ is simple. Then $H$ must be of type $(1,2 ; 2,1 ; 3,2)$ or $(1,6 ; 3,2)$ as an algebra. Otherwise, by Lemma 6.1.1, Remark 6.1.2 and Lemma 6.1.3, there is a Hopf algebra quotient $H \rightarrow \bar{H}$, where $\operatorname{dim} \bar{H}=8$. Therefore $\operatorname{dim} H^{\text {co }} \bar{H}=3$. Decomposing $H^{\mathrm{co}} \bar{H}$ as a direct sum of irreducible left coideals of $H$ (of dimension 1 or 3 ), we find that $H^{\text {co } \bar{H}} \subseteq k G(H)$ and thus $H^{\text {co } \bar{H}}=k T \simeq k^{T}$ is a Hopf subalgebra, where $T$ is the unique subgroup of $G(H)$ of order 3 ; this implies that $H$ is not simple.

Therefore, there is a quotient Hopf algebra $\pi: H \rightarrow A$, where $A$ is of dimension 6 . We have $\operatorname{dim} H^{\mathrm{co} \pi}=4$, implying that $H^{\mathrm{co} \pi}=k 1 \oplus V$, where $V$ is an irreducible left coideal of dimension 3. It follows that the restriction $\left.\pi\right|_{k G(H)}: k G(H) \rightarrow A$ is an isomorphism. In particular, $A \simeq k G$ and $H \simeq R \# k G$ is a biproduct, where $G=G(H)$. The lemma follows from Proposition 4.4.6.

LEMma 6.1.5. Suppose that $H$ is simple. If $H$ has a Hopf subalgebra of dimension 8 , then $H$ is of type $(1,2 ; 2,1 ; 3,2)$ as an algebra.

Proof. By assumption, there is a Hopf algebra quotient $H^{*} \rightarrow B$, where $\operatorname{dim} B=8$; so that $\operatorname{dim}\left(H^{*}\right)^{\operatorname{co} B}=3$ and thus $\left(H^{*}\right)^{\operatorname{co} B}=k 1 \oplus$ $V$ as a left coideal of $H^{*}$, where $V$ is an irreducible left coideal of dimension 2.

Suppose that $H$ is not of the claimed type as an algebra. In view of Lemma 6.1.1 and the previous lemmas, there is a Hopf subalgebra $A \subseteq H^{*}$, with $\operatorname{dim} A=8$. We have $A \cap\left(H^{*}\right)^{\text {co } B}=k 1$, unless $V \subseteq$ $A$. However, the last possibility implies that $\left(H^{*}\right)^{\text {co } B} \subseteq A$, which contradicts Lemma 1.3.4. Therefore, $H^{*}=R \# A$ is a biproduct, where $R$ is a 3-dimensional Yetter-Drinfeld Hopf algebra over $A$.

By Corollary 4.3.4 and Proposition 4.4.6, $H$ is not simple in this case. Thus $H$ is of type $(1,2 ; 2,1 ; 3,2)$, as claimed.

Corollary 6.1.6. Suppose that $H$ is of coalgebra type $(1,8 ; 2,4)$ or $(1,8 ; 4,1)$. Then $H$ is not simple.

Proof. In both cases we have $|G(H)|=8$. By Lemma 6.1.5 and Remark 6.1.2 (ii) we may assume that there is a Hopf algebra quotient $q: H \rightarrow B$, where $\operatorname{dim} B=6$. Since $\operatorname{dim} H^{\operatorname{co} B}=4$, then $\operatorname{dim} H^{\operatorname{co} B} \cap$ $k G(H)=2$ or 4 . The last possibility implies that $H$ is not simple, and 
the first one implies that $\operatorname{dim} q(k G(H))=4$ which contradicts [NZ]. This proves the corollary.

LEMMA 6.1.7. There exists no semisimple Hopf algebra with coalgebra type $(1,4 ; 2,1 ; 4,1)$.

Proof. Suppose on the contrary that $H$ is of type $(1,4 ; 2,1 ; 4,1)$ as a coalgebra. The set of irreducible characters of $H^{*}$ consists of $G(H)$, one irreducible character $\lambda$ of degree 2 and one irreducible character $\psi$ of degree 4. Then $G(H)$ and $\lambda$ span a standard subalgebra of $R\left(H^{*}\right)$, which corresponds to the Hopf subalgebra $B \subseteq H$, with $\operatorname{dim} B=8$. By [M4], $B$ is isomorphic to $H_{8}$ or to $k^{G}$, where $G$ is either the dihedral or the quaternionic group of order 8.

It is not hard to see that the fusion rules in $R\left(H^{*}\right)$ are determined by $\lambda^{2}=\sum_{g \in G(H)} g, \psi^{2}=\sum_{g \in G(H)} g+2 \lambda+2 \psi$; in particular, we have $g \psi=\psi=\psi g$, for all $g \in G(H)$, and $\lambda \psi=2 \psi=\psi \lambda$.

Claim 6.1.8. B is commutative.

Proof. Let $C \subseteq H$ be the 16-dimensional simple subcoalgebra corresponding to $\psi$. By Remark 3.2.2 the fusion rules for $\widehat{H^{*}}$ imply that $B C=C=C B$. Let $\bar{C}=C / C B^{+}$; so that $\bar{C}$ is a cocommutative coalgebra of dimension 2 .

By Corollary 3.2.5 there is a bijective correspondence between simple $\bar{C}$-comodules and simple $B_{\alpha}$-modules, for some invertible normalized 2-cocycle $\alpha: B \otimes B \rightarrow k$.

Suppose that $B$ is not commutative; so that $B \simeq H_{8}$ is the unique nontrivial semisimple Hopf algebra of dimension 8. It follows from [M5, Theorem 4.8 (1)] that any Galois object of $B$ is trivial; so that $B_{\alpha} \simeq B$ as $B$-comodule algebras. This is impossible since $B$ has more than 2 non-isomorphic simple modules. Hence $B$ must be commutative.

We have $B \simeq k^{G}$, where $G$ is not abelian of order 8 . If $B$ is normal in $H$, then $H$ fits into an abelian extension $1 \rightarrow k^{G} \rightarrow H \rightarrow k F \rightarrow 1$, where $F$ is the cyclic group of order 3 .

Consider the associated matched pair $\triangleright: G \times F \rightarrow F, \triangleleft: G \times F \rightarrow$ $G$; see $[\mathbf{M}]$. There exists a subgroup $G_{0}$ of $G$, with $\left|G_{0}\right|=4$, which acts trivially on $F$. The compatibility conditions between $\triangleright$ and $\triangleleft$ imply that $G_{0}$ is stable under the action of $F$. This implies, in view of formulas (4.2) and (4.5) in $[\mathbf{M}]$, that the subspace $A_{0}=k^{F} \otimes k G_{0}$ is a Hopf subalgebra of $H^{*}$ of dimension 12, necessarily normal.

We have $H \simeq k^{G} \#_{\sigma} k F$ is a crossed product, and the irreducible $H$-modules are of dimension 1 or 3 . We may assume $H$ is not commutative, since otherwise $H^{*}=k(F \rtimes G)$ would have no irreducible 
modules of dimension 4. By Lemma $6.1 .1, H^{*}$ is of type $(1,6 ; 3,2)$ as a coalgebra. Hence $A_{0}$ is commutative, and $H^{*}$ fits into an abelian extension $1 \rightarrow A_{0} \rightarrow H^{*} \rightarrow k \mathbb{Z}_{2} \rightarrow 1$. Then the irreducible $H$-comodules are of dimension 1 or 2 . In particular, $H^{*}$ cannot have irreducible modules of dimension 4 . This shows that $B$ is not normal in $H$.

Claim 6.1.9. $H$ is of type $(1,2 ; 2,1 ; 3,2)$ as an algebra.

Proof. Since $B$ is not normal in $H$, then $\left(H^{*}\right)^{\text {co } B^{*}}=k 1 \oplus V$, where $V$ is an irreducible left coideal of dimension 2. Suppose $H$ is not of type $(1,2 ; 2,1 ; 3,2)$. Combining Theorem 2.5.1 with Lemma 6.1 .1 , we see that the possible coalgebra types for $H^{*}$ are $(1,4 ; 2,5)$ and $(1,8 ; 2,4)$.

By Remark 6.1.2, $H^{*}$ contains a Hopf subalgebra $K$ of dimension 8 . By Lemma 1.3.4, $K \cap\left(H^{*}\right)^{\text {co } B^{*}}=k 1$. Hence $K \simeq B^{*}$ is cocommutative and $H^{*}$ is a biproduct $H^{*} \simeq R \# K$, where $\operatorname{dim} R=3$.

By Lemma 4.4.4, there exists a normal subgroup $1 \neq N \subseteq G(K)$ such that $N$ commutes with $R$. Since $|G(K)|=8, N \subseteq Z(G(K))$; so that $N$ commutes also with $K$. Therefore $N$ is central in $H^{*}$, and $H^{*}$ has a central group-like element of order 2. This is a contradiction, since $H$ has no Hopf subalgebra of dimension 12 . Hence $H$ is of type $(1,2 ; 2,1 ; 3,2)$ as an algebra, as claimed.

The irreducible characters of degrees 1 and 2 belong to a commutative Hopf subalgebra $A \subseteq H^{*}$.

Claim 6.1.10. A is normal in $H^{*}$.

Proof. Consider the projection $q: H^{*} \rightarrow k^{G(H)}$. Then we have $\operatorname{dim}\left(H^{*}\right)^{\operatorname{co} q}=6$. Suppose that $A$ is not normal. Then $q$ is not normal, and $G\left(H^{*}\right) \cap\left(H^{*}\right)^{\text {co } q}=1$. Let $1 \neq g \in G\left(H^{*}\right)$. Thus there exists $s \in G(H)$ such that $\langle g, s\rangle \neq 1$. Therefore, we must have $H^{\text {co } A^{*}}=$ $k 1 \oplus k t \oplus V$, where $V$ is an irreducible left coideal of dimension 2. In particular, $H^{\text {co } A^{*}}=B^{\text {co } A^{*}} \subseteq B$.

Since $[H: B]=3$, it follows from Lemma 2.3.2 and Proposition 2.3.1 that $G\left(D(H)^{*}\right) \neq 1$; hence $H$ has a one dimensional YetterDrinfeld module.

Note that $G\left(H^{*}\right) \cap Z\left(H^{*}\right)=1$ and $G(H) \cap Z(H)=1$, since neither $H$ nor $H^{*}$ contain Hopf subalgebras of dimension 12 .

By Lemma 1.6.1, a one dimensional Yetter-Drinfeld module of $H$ is of the form $V_{a, g}$, for some $1 \neq a \in G(H)$. Consider the projection $q: H \rightarrow k^{\langle g\rangle}$. Since $B$ is commutative, $a^{-1} h a=h$, for all $h \in B^{\operatorname{co} q}$. By Theorem 1.6.4, $B^{\mathrm{co} q}$ is a Hopf subalgebra of $B$, which is a contradiction. 
The above claim implies that $H^{*}$ fits into the abelian extension

$$
1 \rightarrow k^{G} \rightarrow H^{*} \rightarrow k F \rightarrow 1,
$$

where $G \simeq \mathbb{S}_{3}$ and $F \simeq \mathbb{Z}_{2} \times \mathbb{Z}_{2}$.

Then $H^{*}$ is isomorphic to a crossed product $k^{G} \#_{\sigma} k F$ as an algebra. Using Clifford theory, we know that the isomorphism classes of irreducible modules of $H^{*}$ are exactly the classes of the modules

$$
V_{x, \rho}:=\operatorname{Ind}_{k^{G} \# k_{\alpha} F_{x}}^{H^{*}} x \otimes \rho,
$$

where $x$ runs over a system of representatives of the action of $F$ in $G$, $F_{x} \subseteq F$ is the stabilizer of $x$, and $\rho$ is an irreducible representation of a twisted group algebra $k_{\alpha} F_{x}$.

We have $\operatorname{dim} V_{x, \rho}=\operatorname{dim} \rho\left[F: F_{x}\right]$. The assumption on the coalgebra structure of $H$ implies that $\operatorname{dim} V_{x, \rho}=4$, for some $x \in G$. This implies that $\left[F: F_{x}\right]=4$ (because we cannot have $\left[F: F_{x}\right]=\operatorname{dim} \rho=2$ ). Hence, the orbit of $x$ has 4 elements, and there must exist $1 \neq y \in G$ such that $F_{y}=F$. This implies that $|G(H)|=8$ and gives a contradiction. This contradiction shows that $H$ cannot have this coalgebra structure and finishes the proof of the lemma.

Lemma 6.1.11. Assume that $H$ is of type $(1,2 ; 2,1 ; 3,2)$ as a coalgebra. Then $H$ is not simple.

Proof. Suppose on the contrary that $H$ is simple. By previous lemmas, $H^{*}$ is of type $(1,4 ; 2,5)$ or $(1,2 ; 2,1 ; 3,2)$ as a coalgebra.

Claim 6.1.12. $H^{*}$ is of type $(1,4 ; 2,5)$ as a coalgebra.

Proof. Suppose not. Then $H^{*}$ is of type $(1,2 ; 2,1 ; 3,2)$.

Hence, there is a Hopf algebra quotient $q: H \rightarrow B$, where $\operatorname{dim} B=$ 6 and $B$ is cocommutative; in particular, $\operatorname{dim} H^{\text {co } B}=4$. On the other hand, $H$ contains a unique Hopf subalgebra $A$ of dimension 6 which is not cocommutative and such that $G(H) \subseteq A$.

As a left coideal of $H, H^{\text {co } B}=k G(H) \oplus V$, where $V$ is an irreducible left coideal of dimension 2, or else $H^{\text {co } B}=k 1 \oplus W$, where $W$ is an irreducible left coideal of dimension 3.

The first possibility implies $H^{\text {co } B} \subseteq A$, which contradicts Lemma 1.3.4. The second possibility implies that $A \cap H^{\mathrm{co} B}=k 1$ and therefore the restriction $\left.q\right|_{A}: A \rightarrow B$ is an isomorphism. This is impossible, since $A$ is not cocommutative. Hence the claim follows.

By Remark 6.1.2 (iii), there is a Hopf subalgebra $B \subseteq H^{*}$ of coalgebra type $(1,4 ; 2,1)$. 
Since $\left[H^{*}: B\right]=3$, it follows from Lemma 2.3.2 and Proposition 2.3.1, $G\left(D(H)^{*}\right) \neq 1$. By Lemma 1.6.1, this is the form $V_{g, \eta}$, for some $1 \neq g \in G(H)$ and $1 \neq \eta \in G\left(H^{*}\right)$.

Consider the projection $q: H \rightarrow k^{\langle\eta\rangle}$. Since $A$ is commutative, $g^{-1} a g=a$, for all $a \in A^{\operatorname{co} q}$. By Theorem 1.6.4, $A^{\operatorname{co} q}$ is a Hopf subalgebra of $A$. This implies that $A^{\operatorname{co} q}=A \subseteq H^{\operatorname{co} q}$. In particular, $\left.\eta\right|_{A}=\epsilon$, and this $\eta$ is the only group-like element of $H^{*}$ with this property: otherwise, $A=H^{\operatorname{co} G\left(H^{*}\right)}$ and $H$ is not simple.

Necessarily, $\left(H^{*}\right)^{\text {co } A^{*}}=k 1 \oplus k s \oplus U$, where $U$ is an irreducible left coideal of $B$ of dimension 2. It follows from Corollary 3.5.2, that $s \in G(B) \cap Z(B)$. Also, $\left.s\right|_{A}=\epsilon$; therefore $s=\eta$.

On the other hand, $\left(H^{*}\right)^{\operatorname{co} A^{*}} \subseteq\left(H^{*}\right)^{\operatorname{co} G(H)}$. Since $B$ cannot be contained in $\left(H^{*}\right)^{\operatorname{co} G(H)}$ by $[\mathbf{N Z}]$, we see that $B^{\operatorname{co} G(H)}=B^{\text {co } A^{*}}=$ $k 1 \oplus k s \oplus U$.

By Remark 1.6.2, $V_{s, g}$ is a Yetter-Drinfeld module of $H^{*}$. Since $s \in Z(B)$, applying again Theorem 1.6.4, we see that $B^{\operatorname{co} G(H)}$ is a Hopf subalgebra of $B$. This is a contradiction, because of the decomposition of $B^{\mathrm{co} G(H)}$ as a left coideal of $H^{*}$. This contradiction finishes the proof of the Lemma.

\subsection{Upper and lower semisolvability}

Our aim in this section is to prove that semisimple Hopf algebras of dimension 24 are both upper and lower semisolvable. We first need the following lemma:

Lemma 6.2.1. Suppose that $H$ is not simple. Then $H$ is upper and lower semisolvable.

Proof. We shall show that $H$ is lower semisolvable, and the other statement will follow by duality. Note that every semisimple Hopf algebra of dimension less than 24 is upper and lower semisolvable; see Table 1. Therefore if $H$ has a normal quotient $H \rightarrow \bar{H}$, where $\bar{H}$ is commutative or cocommutative, then $H$ is lower semisolvable. In particular, we may assume that $G\left(H^{*}\right) \cap Z\left(H^{*}\right)=1$. This, combined with Lemma 6.1 .3 and Remark 6.1 .2 (i), allows us to suppose that $H$ is neither of type $(1,3 ; 2,3 ; 3,1)$ nor $(1,12 ; 2,3)$ as a coalgebra.

By assumption, $H$ fits into an extension

$$
1 \longrightarrow A \longrightarrow H \stackrel{\pi}{\longrightarrow} \bar{H} \longrightarrow 1,
$$

and we may assume that $\bar{H}$ is not trivial. Thus $\operatorname{dim} \bar{H}=8$ or 12 , and $\operatorname{dim} A=3$ or 2 , respectively.

In the first case, we may suppose that $H$ is of type $(1,6 ; 3,2)$ as a coalgebra, by Lemma 6.1.1. In particular, $G(H)$ has a unique (normal) 
subgroup $G$ of order 3, which must coincide with the stabilizer of all simple subcoalgebras of dimension 9 , such that $A=k G$; Remark 3.2.7 now implies that the quotient $\bar{H}=H / H(k G)^{+}$is cocommutative, and $H$ is semisolvable.

Suppose finally that $\operatorname{dim} \bar{H}=12$ and $\operatorname{dim} A=2$. We observe that if $\left|G\left(H^{*}\right)\right|=12$, then any subgroup of order 3 must be contained in $\left(H^{*}\right)^{\text {co } A^{*}} \simeq \bar{H}^{*}$; thus $3 /\left|G\left(\bar{H}^{*}\right)\right|$ and $\bar{H}^{*}$ is commutative or cocommutative. Hence, we may assume that $\left|G\left(H^{*}\right)\right| \neq 12$.

If $\bar{H}$ is not trivial, then its simple subcoalgebras are of dimension 1 or $4[\mathbf{F}]$. Therefore, by Corollary 3.3.2, we may assume that $H$ contains no simple subcoalgebra of dimension 9; this implies that $|G(H)|=4$ or 8, by Lemma 6.1.1. Also, $4=\left|G\left(\bar{H}^{*}\right)\right|$ divides $\left|G\left(H^{*}\right)\right|$, hence we must only consider the cases $\left|G\left(H^{*}\right)\right|=4$ or 8 .

It turns out in this case, that both $H$ and $H^{*}$ contain Hopf subalgebras of dimension 8. If $B \subseteq H, K \subseteq H^{*}$, with $\operatorname{dim} B=\operatorname{dim} K=8$, then $B \cap H^{\text {co } K^{*}}=k 1$, by Lemma 1.3.4. Hence $H=R \# B$, where $B$ is a Hopf subalgebra of dimension 8 .

If $B$ is not cocommutative, then by Corollary 4.3.4, $H$ has a normal Hopf subalgebra of dimension 12, implying that $G\left(H^{*}\right) \cap Z\left(H^{*}\right) \neq 1$; hence we are done in this case. If $B=k G(H)$ is cocommutative, it follows from the proof of Lemma 4.4 .4 (ii) that $H$ has a normal Hopf subalgebra of dimension 4. Thus $H$ is semisolvable in this case as well. This completes the proof of the lemma.

TheOREm 6.2.2. Let $H$ be a semisimple Hopf algebra of dimension 24. Then $H$ is upper and lower semisolvable.

Proof. It will be enough to show that $H$ is not simple. This follows from Remark 6.1.2 (i), Lemmas 6.1.3, 6.1.4 and Corollary 6.1.6, if $H$ is of type $(1,12 ; 2,3),(1,3 ; 2,3 ; 3,1),(1,6 ; 3,2),(1,8 ; 2,4)$ or $(1,8 ; 4,1)$, respectively. Also, from Lemma $6.1 .11, H$ is not simple if $H$ is of type $(1,2 ; 2,1 ; 3,2)$ as a coalgebra. Hence, we may assume that $H$ is of type $(1,4 ; 2,5)$ as a coalgebra, and by Remark 6.1 .2 (iii), $H$ contains a Hopf subalgebra of dimension 8. Applying Lemma 6.1.5, we find that $H^{*}$ is of type $(1,2 ; 2,1 ; 3,2)$ as a coalgebra, and $H$ is not simple by Lemma 6.1.11. 


\section{CHAPTER 7}

\section{Dimension 30}

In this chapter $H$ will denote a semisimple Hopf algebra of dimension 30 over $k$. By [N $\mathbf{N}$, Theorem 4.6], if $H$ fits into an abelian extension

$$
1 \rightarrow k^{\Gamma} \rightarrow H \rightarrow k F \rightarrow 1
$$

where $\Gamma$ and $F$ are finite groups, then $H$ is trivial.

\subsection{Possible (co)-algebra structures}

We shall assume in this section that $H$ is nontrivial, and reduce the possibilities for its algebra and coalgebra structures.

Lemma 7.1.1. The group $G\left(H^{*}\right)$ of group-like elements in $H^{*}$ is of order 2, 3, 5, 6 or 10. As an algebra, $H$ is of one of the following types:

- $(1,2 ; 2,7)$,

- $(1,3 ; 3,3)$,

- $(1,5 ; 5,1)$,

- $(1,6 ; 2,6)$,

- $(1,10 ; 2,5)$.

Proof. It follows from 1.1 that $n:=\left|G\left(H^{*}\right)\right| \neq 15$, and if $n=$ $3,5,6$ or 10 , these are the only possible algebra types for $H$. Suppose that $n=1$; again by 1.1 , it follows that the set of degrees of nonlinear irreducible representations of $H$ has at least three elements and necessarily $H$ has an irreducible module of degree 2. This is impossible by Corollary 2.2.3. Suppose finally that $n=2$. Then, as an algebra, $H$ must be either of type $(1,2 ; 2,7)$ or $(1,2 ; 2,3 ; 4,1)$. By Theorem 2.2.1, $G[\chi]=G\left(H^{*}\right)$ is of order 2 , for all irreducible character $\chi$ such that $\chi(1)=2$. The second possibility implies, by Theorem 2.4 .2 , that $H$ has a quotient Hopf algebra of dimension $2+4.3=14$, which contradicts $[\mathbf{N Z}]$. This completes the proof of the lemma.

REMARK 7.1.2. (i) Observe that if $H$ is of type $(1,5 ; 5,1)$ as an algebra, the irreducible character of degree 5 is stable under multiplication by $G\left(H^{*}\right)$. Also, if $H$ is of type $(1,3 ; 3,3)$ as an algebra, 
every irreducible character $\psi$ of degree 3 is stable under multiplication by $G\left(H^{*}\right)$ : this can be seen decomposing the product $\psi \psi^{*}$ into irreducibles and using the relation (1.2.3).

In the other cases, after Theorem 2.2.1, all irreducible characters $\chi$ of degree 2 have nontrivial isotropy, i.e., $G[\chi]$ is of order 2 for all such characters. Combining this observation with Corollary 3.3.2 and Remark 3.2.7 we find that, in any case, the quotient coalgebra $H^{*} / H^{*}\left(k G\left(H^{*}\right)\right)^{+}$is cocommutative.

(ii) Suppose that $H$ is of type $(1,10 ; 2,5)$ or $(1,6 ; 2,6)$ as an algebra. Then the group $G\left(H^{*}\right)$ is abelian.

ProOF. By part (i), for all irreducible character $\chi$ of degree 2 we have $G[\chi] \neq 1$. The claim follows from Proposition 1.2.6.

Lemma 7.1.3. Suppose that $H$ is of type $(1,2 ; 2,7)$ as a coalgebra. Then $H$ is commutative.

Proof. Note first that if $G(H) \subseteq Z(H)$, in view of Remark 7.1.2, $H$ fits into an abelian extension $1 \rightarrow k G(H) \rightarrow H \rightarrow H / H(k G(H))^{+} \rightarrow$ 1 , thus implying the lemma, in view of $[\mathbf{N}]$.

By Lemma 7.1.1, $\left|G\left(H^{*}\right)\right| \neq 1$. If $\left|G\left(H^{*}\right)\right|$ is even, the lemma follows from Theorem 4.6.5 and $[\mathbf{N}]$. We may thus assume that $\left|G\left(H^{*}\right)\right|$ is odd.

Consider the projection $q: H \rightarrow B$, where $B=k^{G\left(H^{*}\right)}$. Since the dimension of $B$ is odd and $G(H)$ is of order 2, we must have $G(H) \subseteq$ $H^{\operatorname{co} B}$. Therefore, as a left coideal of $H, H^{\operatorname{co} B}$ decomposes in the form $H^{\text {co } B}=k G(H) \oplus V_{1} \oplus \cdots \oplus V_{m}$, where $V_{i}$ is an irreducible left coideal of $H$ of dimension 2 , for all $i=1, \ldots, m$. For $1 \leq i \leq m$, let $C_{i}$ be the simple subcoalgebra of $H$ containing $V_{i}$. By Remark 7.1.2, we have $g C_{i}=C_{i}=C_{i} g$, for all $g \in G(H)$; in particular, $k G(H) \subseteq k\left[C_{i}\right]$.

Claim 7.1.4. $G(H) \subseteq Z\left(k\left[C_{i}\right]\right)$, for all $i=1, \ldots, m$.

Proof. Observe that $V_{i}$ appears in $H^{\text {co } B}$ with multiplicity at most 2 , and moreover that $V_{i}$ appears with multiplicity exactly 2 if and only if $C_{i} \subseteq H^{\text {co } B}$. Consider first the case where $m\left(V_{i}, H^{\text {co } B}\right)=2$. Then $k\left[C_{i}\right] \subseteq H^{\text {co } B}$, whence $\operatorname{dim} k\left[C_{i}\right]<\operatorname{dim} H$. Then $k\left[C_{i}\right]$ is commutative and the claim follows.

Suppose now that $m\left(V_{i}, H^{\text {co } B}\right)=1$. Let $V=V_{i}$. Note that $g V$ and $V g$ are irreducible left coideals of $H$ isomorphic to $V$, and $g V, V g \subseteq$ $H^{\text {co } B}$. The multiplicity condition on $V$ implies that $g V=V=V g$, and the claim follows in this case from Corollary 3.5.2.

Let $A=k\left[C_{i}: \quad 1 \leq i \leq m\right]$. This is a Hopf subalgebra of $H$, and the claim implies that $k G(H) \subseteq Z(A)$. Hence, we may assume that $A \subsetneq H$. Since also $H^{\text {co } B} \subseteq A$, a dimension argument implies that 
$H^{\operatorname{co} B}=A$ and $A$ is commutative. Thus the map $q$ is normal, and $H$ fits into an abelian extension $1 \rightarrow A \rightarrow H \rightarrow k^{G\left(H^{*}\right)} \rightarrow 1$. This implies the lemma in view of $[\mathbf{N}]$.

Lemma 7.1.5. Suppose that $H$ is of type $(1,3 ; 3,3)$ as a coalgebra. Then $H$ is commutative.

Proof. By Lemma 7.1.3, $H$ is not of type $(1,2 ; 2,7)$ as an algebra. Suppose first that $\left|G\left(H^{*}\right)\right|$ is divisible by 3 . Then, by Lemma $4.1 .9, H$ is isomorphic to a biproduct $H \simeq R \# k G(H)$, where $R$ is cocommutative by Remark 7.1 .2 (i). Then the lemma follows in this case by Proposition 4.6.1 and $[\mathbf{N}]$. Hence we may assume that $H$ is not of type $(1,3 ; 3,3)$ nor $(1,6 ; 2,6)$ as an algebra. Similarly, if $H$ is of type $(1,10 ; 2,5)$ as an algebra, then $H$ fits into an exact sequence $1 \rightarrow k G(H) \rightarrow H \rightarrow$ $k^{G\left(H^{*}\right)} \rightarrow 1$, and $k^{G\left(H^{*}\right)} \simeq H / H(k G(H))^{+}$is cocommutative. Hence the result follows by $[\mathbf{N}]$.

Suppose finally that $H$ is of algebra type $(1,5 ; 5,1)$. Let $C$ be the unique simple subcoalgebra of dimension 25 of $H^{*}$. It is clear that $H^{*}=k[C]$ is generated by $C$ as an algebra. Consider the projection $q$ : $H^{*} \rightarrow k^{G(H)}$; we have $\operatorname{dim}\left(H^{*}\right)^{\operatorname{co} q}=10$. Since $|G(H)|$ and $\left|G\left(H^{*}\right)\right|$ are relatively prime, we have $G\left(H^{*}\right) \subseteq\left(H^{*}\right)^{\operatorname{co} q}$. By dimension restrictions, $\left(H^{*}\right)^{\operatorname{co} q}=k G\left(H^{*}\right) \oplus V$, where $V \subseteq C$ is an irreducible left coideal of dimension 5. Let $g$ be a generator of $G\left(H^{*}\right)$; since $V$ is the only 5-dimensional irreducible coideal contained in $\left(H^{*}\right)^{\operatorname{co} q}$, then we must have $g V=V=V g$. By Corollary 3.5.2 $k G\left(H^{*}\right)$ is normal in $H^{*}$. This implies the claim in this case, in view of $[\mathbf{N}]$, since the quotient Hopf algebra $H^{*} / H^{*}\left(k G\left(H^{*}\right)\right)^{+}$is cocommutative, by Remark 7.1.2 (i). This completes the proof of the lemma.

Lemma 7.1.6. Suppose that $H$ is of type $(1,6 ; 2,6)$ as an algebra. Then $H$ is of type $(1,6 ; 2,6)$ as a coalgebra.

Proof. Suppose not. It follows from Lemmas 7.1.1, 7.1.3 and 7.1.5, that $G(H)$ has a subgroup $T$ of order 5 . Since $\left|G\left(H^{*}\right)\right|=6$, we have a sequence of Hopf algebra maps

$$
k T \stackrel{\iota}{\longrightarrow} H \stackrel{\pi}{\longrightarrow} k^{G\left(H^{*}\right)} .
$$

In particular, $\pi(a)=1$, for all $a \in T$, implying that $k T \subseteq H^{\operatorname{co} \pi}$, and since $\operatorname{dim} H^{\mathrm{co} \pi}=\left[H^{*}: k G\left(H^{*}\right)\right]=|T|$, we have $k T=H^{\operatorname{co} \pi}$. Therefore (7.1.7) is an exact sequence of Hopf algebras. By Remark 7.1 .2 (ii), $k T$ and $k^{G\left(H^{*}\right)}$ are both commutative and cocommutative; hence the extension is abelian. This implies that $H$ is trivial, against the assumption. This finishes the proof of the lemma. 
Lemma 7.1.8. Suppose that $H$ is of type $(1,5 ; 5,1)$ as a coalgebra. Then $H$ is commutative.

Proof. By Lemmas 7.1.1, 7.1.3, 7.1.5 and 7.1.6, $G\left(H^{*}\right)$ is of order 5 or 10 . Let $\Gamma \subseteq G\left(H^{*}\right)$ be a subgroup of order 5 , and consider the sequence of Hopf algebra maps $k G(H) \stackrel{\iota}{\longrightarrow} H \stackrel{\pi}{\longrightarrow} k^{\Gamma}$. Then $\operatorname{dim} H^{\mathrm{co} \pi}=\left[H^{*}: k \Gamma\right]=6$ and $k G(H) \cap H^{\mathrm{co} \pi}=k 1$ by $[\mathbf{N Z}]$. Then the composition $\pi \iota: k G(H) \rightarrow k^{\Gamma}$ is an isomorphism, and therefore $H$ is isomorphic to a biproduct $R \# k G$, where $G=G(H)$ is a group of order 5 and $R$ is a semisimple Hopf algebra of dimension 6 in the category of Yetter-Drinfeld modules over $G$.

In particular, $R \simeq H / H(k G(H))^{+}$as a coalgebra, and thus $R$ is cocommutative by Remark 7.1.2 (i). By Proposition 4.6.1, $H$ fits into an abelian extension and the result follows from $[\mathbf{N}]$.

Lemma 7.1.9. Suppose that $H$ is of type $(1,10 ; 2,5)$ as a coalgebra. Then $H$ is commutative.

Proof. By previous lemmas $H$ is, as an algebra, necessarily of type $(1,10 ; 2,5)$. In view of Lemma 4.1 .9 , this implies that $G(H) \simeq$ $G\left(H^{*}\right)$ are abelian and $H$ is isomorphic to a biproduct $R \# k G(H)$, where $\operatorname{dim} R=3$. Thus $R$ is cocommutative and commutative, and since the action of $G(H)$ on $R$ is by coalgebra automorphisms, we have that the unique subgroup of order $5, \Gamma$, of $G(H)$ acts trivially on $R$.

Then $\Gamma \subseteq Z(H)$, and there is an exact sequence $1 \rightarrow k \Gamma \rightarrow H \rightarrow$ $\bar{H} \rightarrow 1$, where $\operatorname{dim} \bar{H}=6$. Note that $\bar{H}$ is cocommutative; otherwise $\bar{H} \simeq k^{F}$, where $F$ is the only non-abelian group of order 6 . But this implies that $F=G\left(\left(k^{F}\right)^{*}\right)$ is a subgroup of $G\left(H^{*}\right)$, which is a contradiction. The lemma follows from $[\mathbf{N}]$.

\subsection{Classification}

In this section we aim to give a proof of Theorem 2. For this, we shall consider the possible structures given by Lemma 6.1.1.

Proof of Theorem 2. By Lemmas 7.1.1, 7.1.2, 7.1.8 and 7.1.9, we may assume that $H$ and $H^{*}$ are both of type $(1,6 ; 2,6)$ as coalgebras. In view of Remark 7.1.2 (ii), the group $G(H)$ is cyclic.

Let $F$ be the unique subgroup of order 2 of $G(H)$. By Remark 7.1.2 (i) all irreducible characters of degree 2 are stable under multiplication by $F$, and therefore the quotient coalgebra $H / H(k F)^{+}$is cocommutative. Clearly, $H$ is a biproduct $R \# k F$, and $R \simeq H / H(k F)^{+}$is a cocommutative coalgebra. This implies that $H$ fits into an abelian extension in view of Proposition 4.6.1, and $H$ is trivial by [N]. 


\section{CHAPTER 8}

\section{Dimension 36}

\subsection{Reduction of the problem}

Let $H$ be a nontrivial semisimple Hopf algebra of dimension 36 over $k$.

LEMma 8.1.1. The order of $G\left(H^{*}\right)$ is either $2,3,4,6,9,12$ or 18 and as an algebra $H$ is of one of the following types:

- $(1,2 ; 2,4 ; 3,2)$,

- $(1,3 ; 2,6 ; 3,1)$,

- $(1,4 ; 2,8)$,

- $(1,4 ; 2,4 ; 4,1)$,

- $(1,4 ; 4,2)$,

- $(1,6 ; 2,3 ; 3,2)$,

- $(1,9 ; 3,3)$,

- $(1,12 ; 2,6)$,

- $(1,18 ; 3,2)$.

By $[\mathbf{G N}]$ there is a simple Hopf algebra $\mathcal{H}$ with algebra and coalgebra type $(1,4 ; 2,4 ; 4,1)$. By construction, $\mathcal{H}=(k G)_{\phi}$ is a twisting of the group algebra of $G=D_{3} \times D_{3}$ with respect to a non-degenerate 2-cocycle $\phi \in \mathbb{Z}_{2} \times \mathbb{Z}_{2}$. Moreover, $\mathcal{H}$ is the only twisting of a group of order 36 that is simple, and we have $\mathcal{H} \simeq \mathcal{H}^{* \operatorname{cop}}[\mathbf{G N}, 4.3]$.

Proof. It follows from 1.1 that $G\left(H^{*}\right)=1$ is impossible. It follows as well that the only possibilities for the algebra types are the prescribed ones if $\left|G\left(H^{*}\right)\right|$ equals either 3, 4, 6, 9, 12 or 18 .

Finally, if $\left|G\left(H^{*}\right)\right|=2$, a counting argument gives that $H$ must be of type $(1,2 ; 2,4 ; 3,2)$ or $(1,2 ; 3,2 ; 4,1)$. In the last case, $H$ has two characters of degree $1, \epsilon$ and $g$, two irreducible characters of degree 3 , $\psi_{1}$ and $\psi_{2}$, and one irreducible character of degree $4, \zeta$. Since $G\left[\psi_{i}\right]=1$, because $G\left(H^{*}\right)$ is of order 2 , then $g \psi_{1}=\psi_{2}$; on the other hand, $g \zeta=$ $\zeta$ because $\zeta$ is the only irreducible character of degree 4 . Counting dimensions, we obtain $\psi_{i} \psi_{i}^{*}=\epsilon+2 \zeta, \quad i=1,2$. Thus $m\left(\psi_{i}, \zeta \psi_{i}\right)=$ $m\left(\zeta, \psi_{i} \psi_{i}^{*}\right)=2$, and therefore

$$
\zeta \psi_{1}=2 \psi_{1}+r \psi_{2}+s \zeta=g \zeta \psi_{1}=2 \psi_{2}+r \psi_{1}+s \zeta
$$


implying that $r=2$ and $\zeta \psi_{1}=2 \psi_{1}+2 \psi_{2}=\zeta \psi_{2}$.

Since $*$ fixes $\zeta$ and permutes the set $\left\{\psi_{1}, \psi_{2}\right\}$, and also since $\psi_{i} \zeta=$ $\left(\zeta \psi_{i}^{*}\right)^{*}$, we find that $m\left(\zeta, \psi_{i} \zeta\right)=0, i=1,2$. But $m\left(\zeta, \psi_{i} \zeta\right)=m\left(\psi_{i}, \zeta \zeta^{*}\right)$, so that $\zeta \zeta^{*}$ decomposes in the form $\zeta \zeta^{*}=\epsilon+g+r \zeta, \quad r \in \mathbb{Z}^{+}$. Taking degrees we see that this is impossible. This discards the possibility $(1,2 ; 3,2 ; 4,1)$ for the algebra type of $H$ and finishes the proof of the lemma.

Remark 8.1.2. (i) Suppose that $H$ is of type $(1,18 ; 3,2)$. Then, by Corollary 1.4.3, $G\left(H^{*}\right) \cap Z\left(H^{*}\right) \neq 1$.

(ii) Suppose that $H$ is of type $(1,2 ; 2,4 ; 3,2)$ as a coalgebra. Since $|G(H)|=2, H$ contains no Hopf subalgebra of dimension 12. By Remark 2.4.3 (i), the irreducible characters of degrees 1 and 2 give rise to a Hopf subalgebra of dimension 18. Therefore, in view of Corollary 1.4.3, $G\left(H^{*}\right) \cap Z\left(H^{*}\right) \neq 1$.

(iii) Suppose that $H$ is of type $(1,6 ; 2,3 ; 3,2)$ as a coalgebra. Then for every irreducible character $\chi$ of degree 2 , we must have $|G[\chi]|=2$ (because there are only 3 such characters) and $H$ has no simple subcoalgebra of dimension 16. Hence, also here, the irreducible characters of degrees 1 and 2 give rise to a Hopf subalgebra of dimension 18 . Therefore $G\left(H^{*}\right) \cap Z\left(H^{*}\right) \neq 1$.

(iv) If $H$ is of type $(1,4 ; 4,2)$ as a coalgebra, then $H$ does not contain any left coideal subalgebra of dimension 3. Therefore $H^{*}$ contains no Hopf subalgebra of dimension 12.

Lemma 8.1.3. Suppose that $H$ is of type $(1,3 ; 2,6 ; 3,1)$ as a coalgebra. Then $H$ is not simple.

Proof. By Theorem 2.2.1, $H$ has a unique Hopf subalgebra $A$ of dimension 12 , which contains $G(H)$ and the unique simple subcoalgebra of dimension 9 of $H$.

The classification of semisimple Hopf algebras of dimension $12[\mathbf{F}]$ implies that $A$ is commutative. Therefore $\operatorname{dim} V \leq[H: A]=3$ for all irreducible $H$-module $V$; see for instance Corollary 3.9 in [AN2]. Then, by the previous remark, we may assume that $H^{*}$ is, as a coalgebra, of one of the types $(1,3 ; 2,6 ; 3,1),(1,4 ; 2,8),(1,9 ; 3,3)$ or $(1,12 ; 2,6)$.

Suppose that $H^{*}$ is of type $(1,9 ; 3,3)$ as a coalgebra. We have a Hopf algebra projection $q: H^{*} \rightarrow A^{*}$, such that $\operatorname{dim} H^{* \text { co } A^{*}}=3$. This projection is necessarily normal, thus $H$ is not simple in this case.

If $H^{*}$ is of type $(1,3 ; 2,6 ; 3,1)$ as a coalgebra, then also $H^{*}$ contains a commutative Hopf subalgebra $B \simeq A$ of dimension 12 . We have a Hopf algebra projection $q: H \rightarrow B^{*}$, for which $\operatorname{dim} H^{\text {co } B^{*}}=3$. Then we may assume $A \cap H^{\text {co } B^{*}}=k 1$ and the restriction $\left.q\right|_{A}: A \rightarrow B^{*}$ 
is an isomorphism. Thus $A$ is cocommutative, which is absurd. This contradiction discards this type.

Similarly, if $H^{*}$ is of type $(1,12 ; 2,6)$ as a coalgebra, then $H$ is a biproduct $H=R \# A$, where $R$ is a braided Hopf algebra over $A$ of dimension 3. Since the irreducible $A$-comodules are of dimension 1 or 3 , we must have $\rho(R) \subseteq k G(A) \otimes R$. This implies that $R$ is a braided Hopf algebra over $k G(A)$ and the biproduct $K=R \# k G(A)$ is a Hopf subalgebra of $H$. But $\operatorname{dim} K=9$ and $K$ is thus cocommutative, which contradicts $|G(H)|=3$. Thus this type is not possible.

Finally suppose that $H^{*}$ is of type $(1,4 ; 2,8)$ as a coalgebra. Consider the surjective Hopf algebra map $H^{*} \rightarrow A^{*}$, so that $\operatorname{dim}\left(H^{*}\right)^{\text {co } A^{*}}=$ 3 and we may assume that $\left(H^{*}\right)^{\text {co } A^{*}}=k 1 \oplus V$ as a left coideal of $H^{*}$, where $V$ is an irreducible coideal of dimension 2. By Theorem 2.5.1 $H^{*}$ contains a Hopf subalgebra $B$ of dimension 12 such that $B$ is not cocommutative. As in the previous paragraphs, it turns out that $A \simeq B^{*}$, which is a contradiction. This finishes the proof of the lemma.

Lemma 8.1.4. Suppose that $H$ is of type $(1,9 ; 3,3)$ as a coalgebra. Then $H$ is not simple.

Proof. If $\left|G\left(H^{*}\right)\right|=9$, then $H=R \# k G(H)$ is a biproduct and the lemma follows from Proposition 4.4.6. Thus, by Remark 8.1.2 and Lemma 8.1.3, we may assume that $\left|G\left(H^{*}\right)\right|=12$ or 4 . In any case, there is a quotient $H \rightarrow k \Gamma$, where $\Gamma$ is a group of order 4 , for which necessarily $k G(H)=H^{\text {co } k \Gamma}$. Hence $H$ is not simple.

Lemma 8.1.5. Suppose that $H$ is simple. Then the order of $G\left(H^{*}\right)$ equals either 4 or 12 . Moreover, $H$ is isomorphic to a biproduct $H \simeq$ $R \# k G$, where $G$ is a group of order 4 and $R$ is a Yetter-Drinfeld Hopf algebra over $G$ of dimension 9 .

Proof. By Remark 8.1.2 and Lemmas 8.1.3 and 8.1.4, $\left|G\left(H^{*}\right)\right|$ is not $18,9,6,3$ or 2 . Therefore, by Lemma 8.1.1, $\left|G\left(H^{*}\right)\right|=4$ or 12 .

Let $\Gamma \subseteq G\left(H^{*}\right)$ be a subgroup of order 4. Consider the quotient $p: H \rightarrow k^{\Gamma}$. Then $\operatorname{dim} H^{\mathrm{co} p}=9$. Let $G$ be a subgroup of $G(H)$ of order 4. Then, by [NZ], $k G \cap H^{\mathrm{co} p}=k 1$ and thus the restriction of $p$ gives an isomorphism $k G \rightarrow k^{\Gamma}$. This implies the lemma.

LEMma 8.1.6. Suppose that $H$ is of type $(1,4 ; 2,4 ; 4,1)$ as a coalgebra. Then every irreducible left coideal of dimension 2 in $H$ is contained in a Hopf subalgebra of dimension 12.

Moreover, $H$ contains two Hopf subalgebras $B$ and $B^{\prime}$, which are of type $(1,4 ; 2,2)$ as coalgebras and such that

(i) $B \cap B^{\prime}=k G(H)$ and $G(H) \simeq \mathbb{Z}_{2} \times \mathbb{Z}_{2}$;

(ii) $B \cup B^{\prime}$ generates $H$ as an algebra. 
Proof. Since $H$ contains no irreducible coideals of dimension 3, then for all irreducible $\chi$ such that $\operatorname{deg} \chi=2$, we have $G[\chi] \neq 1$.

Claim 8.1.7. The action of $G(H)$ by left multiplication on the set $X_{2}$ of irreducible characters of degree 2 has two disjoint orbits $\left\{\chi, \chi^{\prime}\right\}$ and $\left\{\psi, \psi^{\prime}\right\}$. In addition, we have $G[\chi]=G\left[\chi^{\prime}\right]$ and $G[\psi]=G\left[\psi^{\prime}\right]$ are distinct subgroups of order 2 of $G(H)$.

Proof. Note that the tensor product $\chi \chi^{\prime}$ is irreducible for some irreducible characters $\chi$ and $\chi^{\prime}$ of degree 2 , since otherwise, there would be a Hopf subalgebra of dimension $4+4.4=20$, which is impossible. Therefore there exist $\chi, \psi \in X_{2}$ such that $G[\chi]$ is not contained in $G[\psi]$; c.f. 2.4.1. Thus $G[\chi]$ and $G[\psi]$ are distinct subgroups of order 2 of $G(H)$. In particular, the group $G(H)$ is isomorphic to $\mathbb{Z}_{2} \times \mathbb{Z}_{2}$.

Since $|G[\chi]|=|G[\psi]|=2$, then the orbits $G(H) \chi$ and $G(H) \psi$ are of order 2. Moreover, since $G\left[\chi^{\prime}\right]=G[\chi]$, for all $\chi^{\prime} \in G(H) \chi$ (because $G[\chi]$ is normal in $G(H)$ ), then $G(H) \chi$ and $G(H) \psi$ are disjoint orbits. This proves the claim.

Claim 8.1.8. We have $\chi^{*}=\chi$, for all $\chi \in R\left(H^{*}\right)$.

ProOf. We have already shown that $a^{2}=1$, for all $a \in G(H)$. If $\chi$ is irreducible of degree 2 , then we have $\chi \chi^{*}=\sum_{a \in G[\chi]} a+\lambda$, for some $\lambda \in X_{2}$ such that $\lambda^{*}=\lambda$. Also $G[\chi] \subseteq G[\lambda]$, which implies that $\lambda \in G(H) \chi$. Hence every orbit has a self-dual element. Moreover, if $g \in G(H)$ is such that $g \lambda=\chi$, then $\chi^{*}=\lambda g$ and therefore $G[\chi]=$ $G[\lambda] \subseteq G\left[\chi^{*}\right]$. Hence $\chi^{*} \in G(H) \chi$. This implies, since each orbit has two elements, that $\chi^{*}=\chi$. Since $\chi$ was arbitrary, the claim follows.

The claim implies the character algebra $R\left(H^{*}\right)$ is commutative.

As before, we have decompositions $\chi^{2}=\chi \chi^{*}=\sum_{a \in G[\chi]} a+\lambda$, where $\lambda \in G(H) \chi$. On the other hand, if $\chi^{\prime} \in G(H) \chi$, say $b \chi=\chi^{\prime}$, then $\chi^{\prime} \chi=b \chi^{2}=b+b a+b \lambda$, and $b \lambda \in G(H) \chi$. Thus, the set $G(H) \cup G(H) \chi$ spans a standard subalgebra of $R\left(H^{*}\right)$ which corresponds to a Hopf subalgebra $A$ of dimension 12 such that $R\left(A^{*}\right)=k(G(H) \cup G(H) \chi$.

The same argument applies to the other orbit and concludes the proof of the lemma.

Lemma 8.1.9. Suppose that $H$ is of type $(1,4 ; 2,8)$ as a coalgebra. Assume in addition that $H$ is simple. Then $H$ contains two Hopf subalgebras $B$ and $B^{\prime}$, which are of type $(1,4 ; 2,2)$ as coalgebras and such that $B$ and $B^{\prime}$ satisfy the conditions (i) and (ii) of Lemma 8.1.6.

Proof. First note that it is enough to show that $H$ contains Hopf subalgebras $B, B^{\prime}$ of dimension 12 such that $B \cap B^{\prime}=k G$. Indeed, in 
this case, a dimension argument implies that necessarily the subalgebra generated by $B$ and $B^{\prime}$ coincides with $H$. On the other hand, this also implies that the group $G=G(H)$ is isomorphic to $\mathbb{Z}_{2} \times \mathbb{Z}_{2}$ : otherwise $G$ would contain a unique subgroup of order 2 , which would be central both in $B$ and in $B^{\prime}([\mathbf{F}])$ and a fortiori in $H$.

By Lemma 8.1.5 $H=R \# k G$, where $G=G(H)$ and $\operatorname{dim} R=9$. Therefore $R$ decomposes in the form $R=k 1 \oplus V_{1} \oplus V_{2} \oplus V_{3} \oplus V_{4}$ as a left coideal of $H$, where $V_{i}$ is an irreducible left coideal of dimension 2 , for all $i=1, \ldots, 4$.

It follows from Corollary 4.2.2 and Proposition 4.2.1 that $V_{i}$ is a subcoalgebra of $R$, for all $i$, and $V_{i}$ is not isomorphic to $V_{j}$, as left coideals of $H$, if $i \neq j$. Also $\left|G\left[\chi_{i}\right]\right|=2$, where $\chi_{i}=\chi_{V_{i}}$. In particular, $\# G \chi_{i}=2$, for all $i$. We have $V_{i} \# k G=C_{i} \oplus C_{i} h$, where $C_{i}$ is the simple subcoalgebra of $H$ containing $V_{i}$ and $C_{i} h \neq C_{i}$. Thus, if $V_{j} \simeq V_{i} h$ then $V_{j} \# k G=V_{i} \# k G$ and $V_{j}=(\mathrm{id} \otimes \epsilon)\left(V_{j} \# k G\right)=V_{i}$. Therefore $\chi_{i}$, $1 \leq i \leq 4$, form a system of representatives of the orbits of the action of $G$ on $X_{2}$ by right multiplication.

The multiplicity-one condition for the decomposition of $R$ implies that $V_{1}, \ldots, V_{4}$ are uniquely determined, and these are the only irreducible left coideals of $H$ contained in $R$. Thus the action of $G$ on $R$ must permute the $V_{i}$ 's.

Claim 8.1.10. Let $1 \leq i \leq 4$. There exists a subgroup $1 \neq S$ of $G$ such that $s . V_{i}=V_{i}$.

Proof. The possible decompositions of $R$ as an $H$-Yetter-Drinfeld submodule of $H$ are the following

$k 1 \oplus X_{1} \oplus X_{2}, \quad k 1 \oplus Y_{1} \oplus Y_{2} \oplus Y_{3} \oplus Y_{4}, \quad k 1 \oplus X_{1} \oplus Y_{1} \oplus Y_{2}, \quad k 1 \oplus Y_{1} \oplus Z$, where $\operatorname{dim} X_{i}=4, \operatorname{dim} Y_{i}=2, \operatorname{dim} Z=6$. Decomposing the $X_{i}$ 's and $Z$ as left coideals of $H$, and using that the action of $G$ permutes the $V_{i}$ 's, the claim follows.

Let $1 \neq S$ be a subgroup of $G$ such that $s . V_{1}=V_{1}$, for all $s \in S$, as in Claim 8.1.10. The action of $S$ must permute either transitively or trivially the set $G\left(V_{1}\right)$.

Suppose first that the action of $S$ on $V_{1}$ is trivial. Let $V_{1}^{\prime}=G \cdot V_{1}$. Then the action of $S$ on $V_{1}^{\prime}$ is also trivial and so is the action of $S$ on the subalgebra $k\left[V_{1}^{\prime}\right]$ generated by $V_{1}^{\prime}$.

Since $k\left[V_{1}^{\prime}\right]$ is a Yetter-Drinfeld Hopf subalgebra of $R, \operatorname{dim} k\left[V_{1}^{\prime}\right]$ is either 3 or 9 . The last possibility implies that the action of $S$ on $R$ is trivial. Therefore, we may assume that $\operatorname{dim} k\left[V_{1}^{\prime}\right]=3$, and then $V_{1}$ is stable under the action of $G$. Thus $B=k\left[V_{1}\right] \# k G$ is a Hopf 
subalgebra of $H$ of dimension 12. Moreover, since the action of $G$ permutes the set $\left\{V_{1}, \ldots, V_{4}\right\}$ and by the above fixes $V_{1}$, we may assume that also $g V_{2} g^{-1}=V_{2}$, for all $g \in G$. Then the Hopf subalgebras $B$ and $B^{\prime}=k\left[V_{2}\right] \# k G$ do the job.

Suppose finally that the action of $S$ on $V_{1}$ is transitive. By Lemma 4.7.1 $\left|G_{V_{1}}\right|=2$. Let $V_{1}^{\prime}:=G_{V_{1}} . V_{1}$ as above; note that $G_{V_{1}^{\prime}}=G_{V_{1}}$. Then, by Lemma 4.3.1, $\widetilde{B}=k\left[V_{1}^{\prime}\right] \# k G_{V_{1}}=k\left[V_{1}^{\prime}\right] \# k G_{V_{1}^{\prime}}$ is a Hopf subalgebra of $H$. If $k\left[V_{1}^{\prime}\right]=R$, then $\widetilde{B}$ is an index-2 (and therefore normal) Hopf subalgebra of $H$. So we may assume that $\operatorname{dim} k\left[V_{1}^{\prime}\right]=3$, implying that $g . V_{1}=V_{1}$ for all $g \in G$. We may also assume that $g . V_{2}=V_{2}$ for all $g \in G$. Let $B=k\left[V_{1}\right] \# k G$ and $B^{\prime}=k\left[V_{2}\right] \# k G$. Then we have $\operatorname{dim} B=\operatorname{dim} B^{\prime}=12$, and we are done.

Lemma 8.1.11. Suppose that $H$ is of coalgebra type $(1,4 ; 2,4 ; 4,1)$ or $(1,4 ; 2,8)$. Assume in addition that $H$ is simple. Then $H \simeq \mathcal{H}$.

Here $\mathcal{H}$ denotes the simple (self-dual) example in [GN].

Proof. Let $B$ and $B^{\prime}$ be the Hopf subalgebras of $H$ as in Lemmas 8.1.6 and 8.1.9, respectively. Then neither $B$ nor $B^{\prime}$ is cocommutative.

Claim 8.1.12. We have $B \simeq B^{\prime} \simeq \mathcal{A}_{0}$; see Chapter 5 .

Proof. Suppose not. Since $G(H) \simeq \mathbb{Z}_{2} \times \mathbb{Z}_{2}$, then $B$ and $B^{\prime}$ are not isomorphic to $\mathcal{A}_{1}$. In virtue of the classification in dimension 12 $[\mathbf{F}]$, at least one of them, say $B$, is commutative. Then there exists $1 \neq g \in Z\left(B^{\prime}\right) \cap G\left(B^{\prime}\right)$. Since $G\left(B^{\prime}\right)=G(B) \subseteq B, g$ also commutes with $B$. Since $B$ and $B^{\prime}$ generate $H$ as an algebra, this implies that $g$ is a central group-like element in $H$, contradicting the assumption.

We know from Chapter 5 that there exists a normalized 2-cocycle $\phi \in k G(H) \otimes k G(H)$ such that $B_{\phi} \simeq B_{\phi}^{\prime} \simeq k G$, where $G$ is the group in Proposition 5.2.1. Moreover, $B_{\phi}$ and $B^{\prime}{ }_{\phi}$ are Hopf subalgebras of $H_{\phi}$ and $B_{\phi} \cup B^{\prime}{ }_{\phi}$ generates $H_{\phi}$ as an algebra, because the multiplication was unchanged. It turns out that $H_{\phi}$ is cocommutative.

More precisely, $H_{\phi}=k G^{\prime}$, where $G^{\prime}=F^{\prime} \rtimes \Gamma$ is a semidirect product with $\left|F^{\prime}\right|=9$ and $\Gamma=G(H)$. Since $H$ is simple by assumption, then by the results in [GN, 4.3], $G \simeq D_{3} \times D_{3}$ and $H \simeq \mathcal{H}$.

LEMma 8.1.13. Suppose that $H$ is of type $(1,12 ; 2,6)$ as a coalgebra. Then $H$ is not simple.

Proof. By Lemma 8.1.5, we may assume that $\left|G\left(H^{*}\right)\right|=4$ or 12 . By Lemma 8.1.11, if $\left|G\left(H^{*}\right)\right|=4$ we may also assume that $H^{*}$ is of type $(1,4 ; 4,2)$ as a coalgebra; this is impossible in view of Remark 
8.1.2 (iv). Therefore, we must consider the case $\left|G\left(H^{*}\right)\right|=12$, i.e., $H$ and $H^{*}$ are both of type $(1,12 ; 2,6)$.

Consider the quotient Hopf algebra $p: H \rightarrow k^{G\left(H^{*}\right)}$. We have $\operatorname{dim} H^{\operatorname{co} p}=3$. If $g \in H^{\operatorname{co} p}$ for some $g \in G(H)$, then by $[\mathbf{N Z}] g$ is of order 3 and $H^{\text {co } p}=k\langle g\rangle$ is a normal Hopf subalgebra of $H$, implying that $H$ is not simple. Thus, we may assume that $p(g) \neq 1$, for all $g \in$ $G(H)$. This implies that the restriction $\left.p\right|_{k G(H)}: k G(H) \rightarrow k^{G\left(H^{*}\right)}$ is an isomorphism. Therefore the groups $G(H)$ and $G\left(H^{*}\right)$ are isomorphic and abelian and $H$ is a biproduct $H=R \# k G(H)$, where $\operatorname{dim} R=3$. The lemma follows from Proposition 4.4.6.

\subsection{Main result}

In view of the previous results, the only case it remains to consider is that where $H$ and $H^{*}$ are both of type $(1,4 ; 4,2)$ as coalgebras.

THEOREM 8.2.1. Let $H$ be a semisimple Hopf algebra of dimension 36. Suppose that $H$ is simple. Then $H \simeq \mathcal{H}$.

Proof. By Lemma 8.1.5, $H$ is a biproduct $H=R \# k G(H)$, where $R$ is a left coideal subalgebra of dimension 9 . Therefore, as a left coideal of $H, R=k 1 \oplus V_{1} \oplus V_{2}$, where $V_{i}$ are irreducible left coideals of dimension $4, i=1,2$.

ClaIm 8.2.2. There exists a normalized invertible 2-cocycle $\phi \in$ $k G(H) \otimes k G(H)$ such that $H_{\phi}$ is not simple.

Proof. We have $g V_{i} \simeq V_{i} \simeq V_{i} g$ as a left coideal of $H$, for all $g \in G(H)$; so that by Corollary $4.2 .2, V_{i}$ is a subcoalgebra of $R$ in the category of Yetter-Drinfeld modules over $G(H)$, and we have $V_{i} \# k G(H)$ is a simple subcoalgebra of dimension 16. It is also clear that $V_{i}$ generates $R$ as an algebra, because $k\left[V_{1}\right]$ is a braided Hopf subalgebra of $R$ containing $V_{i}$; then $\operatorname{dim} V_{i}^{G}=1$ by [N3, Lemma 4.4.1].

Write $G=G(H)$. By $[\mathbf{N 2}, 1.3]$ there exist normalized 2-cocycles $\alpha_{i}: G \times G \rightarrow G$ such that $V_{i} \simeq\left(k_{\alpha_{i}} G\right)^{*}, i=1,2$, and moreover, in view of the results in [N3, Section 4] there exists a normalized invertible 2-cocycle $\phi \in k G \otimes k G$ such that $H_{\phi}=R_{\phi} \# k G$, and $V:=V_{1}$ is a cocommutative subcoalgebra of $R_{\phi}$. See [N3, Remark 4.4.3].

Suppose that $H_{\phi}$ is simple. Then $H_{\phi} \simeq H$ as algebras and as coalgebras, whence $V \# k G(H)$ is a simple subcoalgebra of $H_{\phi}$. The claim can be established using the argument in the proof of $[\mathbf{N 3}$, Theorem 1.0.1]. We sketch this proof below for the sake of completeness.

Write $G(V)=\left\{x_{1}, \ldots, x_{4}\right\}$. By [N3, Lemma 2.2.1] $\left(\mathcal{S}_{R_{\phi}} V\right) V$ is a Yetter-Drinfeld subcoalgebra of $R_{\phi}$, and $\left(\mathcal{S}_{R_{\phi}} V\right) x_{i}$ is a left coideal of $R$ containing 1 , for all $i=1, \ldots, 4$, where $\mathcal{S}_{R_{\phi}}$ is the antipode of $R_{\phi}$. 
Since the dimensions of the irreducible comodules of a twisted dual group algebra of $G$ divide the order of $G$, we have that the dimensions of the irreducible $R_{\phi}$-comodules are either 1 or 2 .

The group $G$ acts on $R_{\phi}$ by $\leftarrow: R_{\phi} \otimes k G \rightarrow R_{\phi}, r<g:=\left(g, r_{-1}\right) r_{0}$, $r \in R_{\phi}$, where ( , ) is a non-degenerate bicharacter on $G$. Moreover, this action restricts to an action of $G$ on $\left(\mathcal{S}_{R_{\phi}} V\right) V$ by coalgebra automorphisms. Let $\omega_{1}, \ldots, \omega_{l}$ be the group-like elements of $R_{\phi}$ which belong to $\left(\mathcal{S}_{R_{\phi}} V\right) V$. Then the action $<$ of $G$ permutes the set $\left\{\omega_{1}, \ldots, \omega_{l}\right\}$. As in [N3, Claim 5.3.1], one can see that every group-like element $\omega$ in $\left(\mathcal{S}_{R_{\phi}} V\right) V$ belongs to $\left(\mathcal{S}_{R_{\phi}} V\right) x_{i}$ for all $1 \leq i \leq n$.

Therefore $\mathcal{S}_{R_{\phi}} V x_{i}=k \omega_{1} \oplus \cdots \oplus k \omega_{l} \oplus \bigoplus_{j} W_{j}$, for all $i$, where $W_{j}$ are irreducible right coideals of $R_{\phi}$ of dimension bigger than 1 . Thus $l$ is even, and since $1 \in\left\{\omega_{1}, \ldots\right\}$, there exists a nontrivial group-like element in $R_{\phi}$ invariant under the action $<$ of $G$; this is exactly the same as a coinvariant group-like element in $R_{\phi}$. Then $G\left(H_{\phi}\right) \cap R_{\phi}$ has an element of order 3 . This is a contradiction, because $G\left(H_{\phi}\right)$ is of order 4. The contradiction comes from the assumption that $H_{\phi}$ is simple, hence the claim follows.

Let $A \subseteq H_{\phi}$ be a proper normal Hopf subalgebra. Since $H_{\phi} \simeq H$ as algebras, $H_{\phi}$ contains no Hopf subalgebras of dimension 12, by Remark 8.1 .2 (iv). Then $\operatorname{dim} A=2,3,4,6$ or 18 . Note that $k G(H)_{\phi}=k G(H)$ is a Hopf subalgebra of $H_{\phi}$; so that 4 divides the order of $G\left(H_{\phi}\right)$. If $\operatorname{dim} A=3$, then either $\left|G\left(H_{\phi}\right)\right|=12$, which contradicts Remark 8.1.2 (iv), or else $H_{\phi}$ is cocommutative. The last possibility implies $H \simeq \mathcal{H}$ by $[\mathbf{G N}, 4.3]$; hence we may assume that $\operatorname{dim} A \neq 3$.

If $\operatorname{dim} A=2$, then $A \subseteq k G\left(H_{\phi}\right) \cap Z\left(H_{\phi}\right)$ by Corollary 1.4.3. Thus $A \subseteq k G(H) \cap Z(H)$; see Corollary 5.4.2. Also, if $\operatorname{dim} A=4$, then there is a quotient Hopf algebra $H_{\phi} \rightarrow H_{\phi} / H_{\phi} A^{+}=: \overline{H_{\phi}}$, such that $\operatorname{dim} \overline{H_{\phi}}=9$. In particular, $9 /\left|G\left(H_{\phi}^{*}\right)\right|$, which is a contradiction.

Suppose that $\operatorname{dim} A=6$. Then, with the above notation, $\operatorname{dim} \overline{H_{\phi}}=$ 6. Hence ${\overline{H_{\phi}}}^{*}$ contains a group-like Hopf subalgebra of dimension 3 or else a simple subcoalgebra of dimension 4 , which again contradicts the fact that $H_{\phi}$ is of type $(1,4 ; 4,2)$ as an algebra.

Finally, if $\operatorname{dim} A=18$, then $|G(A)|=2$ and by [M3] $A$ is commutative. It follows from [AN2, Corollary 3.9], $\operatorname{dim} V \leq\left[H_{\phi}: A\right]=2$, for all irreducible $H_{\phi}$-module $V$. This is impossible since $H_{\phi}$ is of type $(1,4 ; 4,2)$ as an algebra. This finishes the proof of the theorem. 


\section{CHAPTER 9}

\section{Dimension 40}

\subsection{Reduction of the problem}

$k$.

Let $H$ be a nontrivial semisimple Hopf algebra of dimension 40 over

LEMMA 9.1.1. The order of $G\left(H^{*}\right)$ is either 4,8 or 20 and as an algebra $H$ is of one of the following types:

- $(1,4 ; 2,9)$,

- $(1,4 ; 2,5 ; 4,1)$,

- $(1,4 ; 2,1 ; 4,2)$,

- $(1,8 ; 2,8)$,

- $(1,8 ; 2,4 ; 4,1)$,

- $(1,8 ; 4,2)$,

- $(1,20 ; 2,5)$.

We shall prove that the type $(1,4 ; 2,5 ; 4,1)$ is impossible. See Lemma 9.1.5.

ProOf. Let $n=\left|G\left(H^{*}\right)\right|$. It follows from 1.1 that $n \neq 1,5,10$. The possibility $n=2$ is discarded using Theorem 2.2.1. In the cases $n=4,8,20$ the only possibilities excluded are the types $(1,4 ; 3,4)$ and $(1,4 ; 6,1)$; however, in this cases $H$ has an irreducible character $\psi$ of degree 3 (respectively 6 ), and decomposing the product $\psi \psi^{*}$ as a sum of irreducible characters gives a contradiction. This finishes the proof of the lemma.

REMARK 9.1.2. (i) If $H$ is of type $(1,20 ; 2,5)$, then $H$ is not simple, by Corollary 1.4.3.

(ii) Let $H$ be as in Lemma 9.1.1. It follows from Proposition 2.1.3 that there is a Hopf subalgebra $A \subseteq H$, with $\operatorname{dim} A=8$.

Lemma 9.1.3. We have $H=R \# A$ is a biproduct where $A$ is a semisimple Hopf algebra of dimension 8 and $R$ is a 5-dimensional Yetter-Drinfeld Hopf algebra over A.

Proof. There are a Hopf subalgebra $A \subseteq H$ and a quotient Hopf algebra $q: H \rightarrow B$, such that $\operatorname{dim} A=\operatorname{dim} B=8$. In particular, $\operatorname{dim} H^{\operatorname{co} B}=5$, and therefore $G(H) \cap H^{\operatorname{co} B}=1$ by $[\mathbf{N Z}]$. 
Consider the restriction $\left.q\right|_{A}: A \rightarrow B$. It will be enough to show that $q$ is an isomorphism, or equivalently, that $A \cap H^{\text {co } B}=k 1$. Suppose on the contrary that $A \cap H^{\text {co } B}=A^{\text {co } B}$ is of dimension 2 or 4; see Lemma 1.3.4. Then there must exist $1 \neq g \in G(A) \cap A^{\text {co } B}$, since the irreducible left coideals of $A$ are of dimension 1 or 2 . Then $2 / \operatorname{dim} H^{\operatorname{co} B}=5$, which is absurd. This contradiction finishes the proof of the lemma.

LEMMA 9.1.4. Suppose that $H$ is simple and $|G(H)|=4$. Then $R=k 1 \oplus U$, where $U$ is an irreducible left coideal of $H$ of dimension 4. In particular, $H$ is not of type $(1,4 ; 2,9)$ as a coalgebra.

Proof. Since $|G(H)|=4, A$ is not cocommutative. Suppose on the contrary that $R=k 1 \oplus V_{1} \oplus V_{2}$, where $V_{i}$ is an irreducible left coideal of dimension 2. By Lemma 4.3.3 (i), $\rho\left(V_{i}\right) \subseteq k G(H) \otimes V_{i}$, $i=1,2$. Thus $\rho(R) \subseteq k G(H) \otimes R$ and $R \# k G(H)$ is a normal Hopf subalgebra of $H$ (of index 2).

Lemma 9.1.5. There exists no semisimple Hopf algebra $H$ of type $(1,4 ; 2,5 ; 4,1)$ as a coalgebra.

Proof. Suppose on the contrary that $H$ is of type $(1,4 ; 2,5 ; 4,1)$. Let $A \subseteq H$ be a Hopf subalgebra of dimension 8. For all irreducible $H^{*}$-characters $\chi$ such that $\operatorname{deg} \chi=2$, we have that $G[\chi] \neq 1$, because $H$ does not contain irreducible coideals of dimension 3. Also, the tensor product $\chi \chi^{\prime}$ is irreducible for some irreducible characters $\chi$ and $\chi^{\prime}$ of degree 2 , since otherwise, there would be a Hopf subalgebra of dimension $4+4.5=24$, which is impossible. Therefore there exist $\chi, \psi \in X_{2}$ such that $G[\chi]$ is not contained in $G[\psi]$; c.f. Lemma 2.4.1. Thus $G[\chi]$ and $G[\psi]$ are distinct subgroups of order 2 of $G(H)$.

Since $|G[\chi]|=|G[\psi]|=2$, then the orbits $G(H) \chi$ and $G(H) \psi$ are of order 2. Moreover, since $G\left[\chi^{\prime}\right]=G[\chi]$, for all $\chi^{\prime} \in G(H) \chi$ (because $G[\chi]$ is normal in $G(H)$ ), then $G(H) \chi$ and $G(H) \psi$ are disjoint orbits. Let $\tau \notin A$ be an irreducible character of degree 2 . Then we have

$$
\tau \tau^{*}=\sum_{a \in G[\tau]} a+\lambda,
$$

for some $\lambda \in X_{2}$. By (9.1.6), we have $\lambda^{*}=\lambda$ and $G[\tau] \subseteq G[\lambda]$. Therefore, either $\lambda \in G(H) \tau$ or $\lambda \in A$.

Claim 9.1.7. We have $\lambda \in A$.

Proof. Suppose on the contrary that $\lambda \in G(H) \tau$; hence, every orbit has a self-dual element. Moreover, if $g \in G(H)$ is such that $g \lambda=\tau$, then $\tau^{*}=\lambda g$ and therefore $G[\tau]=G[\lambda] \subseteq G\left[\tau^{*}\right]$. Hence $\tau^{*} \in G(H) \tau$. This implies, since each orbit has two elements, that 
$\tau^{*}=\tau$. As before, we have decompositions $\tau^{2}=\tau \tau^{*}=\sum_{a \in G[\tau]} a+\lambda$, where $\lambda \in G(H) \tau$ and if $\tau^{\prime} \in G(H) \tau$, say $b \tau=\tau^{\prime}$, then $\tau^{\prime} \tau=b \tau^{2}=$ $b+b a+b \lambda$, and $b \lambda \in G(H) \tau$. Then the set $G(H) \cup G(H) \tau$ spans a standard subalgebra of $R\left(H^{*}\right)$ which corresponds to a Hopf subalgebra of dimension 12. This contradicts [NZ]; so we may conclude that $\lambda$ is not in the orbit $G(H) \tau$. Therefore, we must have $\lambda \in A$ as claimed.

Since $\chi \psi$ is irreducible, it follows from Lemma 2.4.1 that $m\left(\lambda, \psi^{*} \psi\right)=$ 0. This contradicts Claim 9.1.7. Then the lemma follows.

REMARK 9.1.8. Keep the notation in Lemma 9.1.3.

(i) Suppose that $R=k 1 \oplus U$, where $U$ is an irreducible left coideal of dimension 4 and let $\chi \in H$ be the corresponding character. It follows from Corollary 4.2.2, that $\left|G\left[\chi^{*}\right]\right| \leq 4$.

(ii) If $\left|G\left(H^{*}\right)\right|=|G(H)|=8$, then $H$ is not simple by [N2, Lemma 2.2.5]. So we may assume that $A$ is not a group algebra of an abelian group. In particular, after dualizing if necessary, we may assume that $A$ is not cocommutative.

Lemma 9.1.9. Suppose that $H$ is of type $(1,8 ; 2,4 ; 4,1)$ as a coalgebra. Then $H$ is not simple.

Proof. By Remark 9.1.8 (ii), we may assume that $G(H)$ is not abelian of order 8. Consider the action by right multiplication of $G(H)$ on the set $X_{2}$ of irreducible characters of degree 2. Then either this action is transitive or else $\left|G\left[\chi^{*}\right]\right|=4$, for all $\chi \in X_{2}$.

The first possibility implies, in view of Remark 2.4.3 (iii) that $H$ has a Hopf subalgebra of dimension 24, which is impossible. Consider the second possibility. Since $G(H)$ is not abelian, $1 \neq[G ; G] \subseteq G\left[\chi^{\prime}\right] \cap$ $G\left[\chi^{*}\right]$, for all $\chi^{\prime}, \chi \in X_{2}$. Therefore, by Theorem 2.4.2, also in this case there is a Hopf subalgebra of dimension 24, which is impossible. This proves the lemma.

Lemma 9.1.10. Suppose that $H$ is of type $(1,8 ; 4,2)$ as a coalgebra. Then $H$ is not simple.

Proof. Suppose that $H$ is simple. We have $\operatorname{dim} R\left(H^{*}\right)=10$ and $G(H)$ is not abelian, by Remark 9.1.8 (ii). Let $e_{0}, e_{1}, e_{2}, e_{3}, f_{1}, f_{2}$ be orthogonal primitive idempotents in $k G(H)$ such that $\operatorname{dim} k G(H) e_{j}=$ 1 and $\operatorname{dim} k G(H) f_{j}=2$. Then $\operatorname{dim} H e_{j}=5$ and $\operatorname{dim} H f_{j}=10$, and moreover $H f_{1} \simeq H f_{2}$.

We may assume that $\left|G\left(H^{*}\right)\right| \neq 8$. Then, by Lemma 9.1.4, $R^{*} \simeq$ $k 1 \oplus U$, where $U$ is an irreducible left coideal of $H^{*}$, and a fortiori an irreducible Yetter-Drinfeld submodule of $H^{*}$. 
By Proposition 1.5.2, there is a bijective correspondence between the irreducible Yetter-Drinfeld submodules of $H$ appearing in $R^{*}=$ $\left(H^{*}\right)^{\operatorname{co} k G(H)^{*}}$ and primitive idempotents $e \in R\left(H^{*}\right)$ such that $e e_{0} \neq 0$.

Therefore we have a decomposition $e_{0}=\Lambda+E_{0}$, where $\Lambda \in H$ is the normalized integral and $E_{0}$ is a primitive idempotent of $R\left(H^{*}\right)$ such that $\operatorname{dim} H E_{0}=4$. This gives at least 7 orthogonal idempotents in $R\left(H^{*}\right)$, implying that $R\left(H^{*}\right) \simeq k^{(6)} \times M_{2}(k)$ as an algebra. In particular, since $f_{1}$ and $f_{2}$ are not central in $k G(H)$, they correspond necessarily to the 4-dimensional simple component of $R\left(H^{*}\right)$.

We find that there must exist some $1 \leq j \leq 3$ such that $e_{j}$ is not primitive in $R\left(H^{*}\right)$. By the Kac-Zhu Theorem, this implies the existence of a primitive idempotent $\Lambda \neq E \in R\left(H^{*}\right)$ such that $\operatorname{dim} H E=1$. Then $G\left(H^{*}\right) \cap Z\left(H^{*}\right) \neq 1$ and the lemma follows.

Lemma 9.1.11. Suppose that $H$ is of type $(1,8 ; 2,8)$ as a coalgebra. Then $H$ is not simple.

Proof. We have $H=R \# k G$, where $G=G(H)$ and $R=k 1 \oplus$ $V_{1} \oplus V_{2}$, where $V_{i}$ are irreducible left coideals of $H$ such that $\operatorname{dim} V_{i}=2$. By Theorem 2.2.1 we must have $G[\chi] \neq 1$, for all irreducible characters $\chi$ of degree 2. Then $V_{i}$ is a subcoalgebra of $R$, by Proposition 4.2.1, and $V_{1}$ is not isomorphic to $V_{2}$ as left coideals of $H$. The group $G$ permutes the set $G\left(V_{1}\right) \cup G\left(V_{2}\right)$ and we may also assume that the group homomorphism $G \rightarrow \mathbb{S}_{4}$ is injective. In particular, $G \simeq D_{4}$ and acts transitively on $G\left(V_{1}\right) \cup G\left(V_{2}\right)$.

Let $G_{i}=\left\{g: g . V_{i} \subseteq V_{i}\right\}$. Then $\left|G_{i}\right| \geq 4$ and in particular, $Z(G) \subseteq$ $G_{i}, i=1,2$. By Lemma 4.7.1, if $Z(G)$ acts transitively on $G\left(V_{i}\right)$, then $\left|G_{V_{i}}\right| \leq 2$. Moreover, the action of $Z(G)$ is either transitive or trivial on $G\left(V_{1}\right)$. Observe in addition that $V_{1}$ generates $R$ as an algebra. Hence, the last possibility implies that $Z(G) \subseteq Z(H)$ and $H$ is not simple.

In the other case, we may conclude that $\rho(R) \subseteq k G_{V_{i}} \otimes R$ and since $\left|G_{V_{i}}\right|=2, B=R \# k G_{V_{i}}$ is a Hopf subalgebra of dimension 10. By Remark 9.1.8 (ii), we may assume that $\left|G\left(H^{*}\right)\right|=4$. Then $\left(H^{*}\right)^{\text {co } B^{*}}=$ $k 1 \oplus k t \oplus V$, where $1 \neq t \in G(H)$ and $V$ is an irreducible left coideal of dimension 2. Consider the possible decompositions of $\left(H^{*}\right)^{\text {co } B^{*}}$ as a Yetter-Drinfeld submodule of $H^{*}$, as in Lemma 1.4.1. Since 3 does not divide $\operatorname{dim} H$, the dimensions of the irreducible summands are either 1 or 2. Decomposing each irreducible Yetter-Drinfeld summand as a sum of irreducible left coideals, implies that $k t$ is necessarily a YetterDrinfeld submodule of $H$. Hence $t \in G(H) \cap Z(H)$ and $H$ is not simple. This concludes the proof of the lemma. 


\subsection{Main result}

In view of the lemmas in the previous section, we may assume that $H$ is of type $(1,4 ; 2,1 ; 4,2)$ as an algebra and as a coalgebra. Moreover we have $H=R \# A$, where $A \simeq H_{8}$ and $R=k 1 \oplus U$, for some irreducible left coideal $U$ of dimension 4 . We keep this notation along this section.

LEMMA 9.2.1. If $R$ is cocommutative then $H$ is not simple.

Proof. We have $U \subseteq R$ is a subcoalgebra in ${ }_{A}^{A} \mathcal{Y} \mathcal{D}$ and $\mathcal{S}_{R} U=U$. On the other hand, $U t \simeq U$ for some $1 \neq t \in G(H)=G(A)$. Let $G(U)=\left\{x_{1}, \ldots, x_{4}\right\}$. By [N3, Lemmas 2.2.1 and 2.2.3] $U x_{i}$ is a left coideal of $R$ and $U x_{i} \simeq U\left(t . x_{i}\right)$; so if $t . x_{i} \neq x_{i}$, by counting dimensions, $x_{i} \in U x_{i}$ implying that $1 \in U$, which is a contradiction. Therefore $t$ acts trivially on $R$. The lemma will follow from the following claim:

Claim 9.2.2. Let $A \rightarrow$ End $V$ be an action of $A \simeq H_{8}$ on a vector space $V$ such that there exists $1 \neq t \in G(A)$ with $\left.t\right|_{V}=\mathrm{id}_{V}$. Then $\left.t^{\prime}\right|_{V}=\mathrm{id}_{V}$ for all $t^{\prime} \in G(A) \cap Z(A)$.

Proof. By $[\mathbf{M 4}]$ we know that $A$ is generated as an algebra by $G(A)=\{1, x, y, x y\}$ and an invertible element $z$ such that $z x=y z$. Moreover, $G(A) \cap Z(A)=\{1, x y\}$. If $t=x y$, then there is nothing to prove. Thus, without loss of generality we may assume that $t=x$. In this case the relation $z x=y z$ plus the fact that $z$ is invertible imply that also $\left.y\right|_{V}=\mathrm{id}_{V}$. Then we have $\left.x y\right|_{V}=\mathrm{id}_{V}$ as claimed.

We now prove the main result of this chapter.

TheOREM 9.2.3. Let $H$ be a semisimple Hopf algebra of dimension 40. Then $H$ is not simple.

Proof. We may assume that $H=R \# A$ is a biproduct, where $A \simeq H_{8}$ and $R=k 1 \oplus U$. By Lemma 9.2.1 it will be enough to show that $U$ is cocommutative. Suppose on the contrary that $U$ is a simple coalgebra. Observe that $U \# A$ is a subcoalgebra of $H$; moreover, there exist $t, s \in G(H)$ such that $U t \simeq U$ and $U s$ is an irreducible coideal not isomorphic to $U$. In particular, $(U \# A) \cap C \neq 0$ and $(U \# A) \cap C^{\prime} \neq 0$, where $C$ and $C^{\prime}$ are the simple subcoalgebras of $H$ containing $U$ and $U s$, respectively. Thus, $U \# A=C \oplus C^{\prime} \simeq M_{4}(k) \oplus M_{4}(k)$.

On the other hand, the assumption that $U$ is simple implies that $U \# A \simeq U \otimes_{\phi} A$ for some invertible normalized 2-cocycle $\phi \in A \otimes A$; see $[\text { Mo, 7.3.1]. By [M5, Theorem } 4.8(1)]_{\phi} A \simeq A$ as coalgebras, which gives $U \# A \simeq M_{2}(k)^{(4)} \oplus M_{4}(k)$. This is a contradiction, which implies that $U$ is cocommutative and finishes the proof of the theorem. 


\section{CHAPTER 10}

\section{Dimension 42}

Let $H$ be a semisimple Hopf algebra of dimension 42 over $k$. We shall assume that $H$ is nontrivial. Suppose that $H$ fits into an abelian extension

$$
1 \rightarrow k^{\Gamma} \rightarrow H \rightarrow k F \rightarrow 1,
$$

where $\Gamma$ and $F$ are finite groups. It follows from the results in $[\mathbf{N}$, Section 4] that in this case $H$ is isomorphic to one of the Hopf algebras $\mathcal{A}_{7}(2,3)$ or $\mathcal{A}_{7}(3,2)=\mathcal{A}_{7}(2,3)^{*}$ constructed in $[\mathbf{A N},(2.3 .1)]$; indeed $\mathcal{A}_{7}(2,3)$ (respectively, $\left.\mathcal{A}_{7}(3,2)\right)$ fits into an extension as above, where $\Gamma$ is the non-abelian group of order 14 and $F$ is the cyclic group of order 3, (respectively, $\Gamma$ is the non-abelian group of order 21 and $F$ is the cyclic group of order 2).

For these Hopf algebras, $G\left(\mathcal{A}_{7}(3,2)\right) \simeq G\left(\mathcal{A}_{7}(2,3)\right) \simeq \mathbb{Z}_{6}$. As coalgebras, $\mathcal{A}_{7}(3,2)$ is of type $(1,6 ; 2,9)$ and $\mathcal{A}_{7}(2,3)$ is of type $(1,6 ; 3,4)$.

\subsection{Possible (co)-algebra structures}

Let $H$ be a nontrivial semisimple Hopf algebra of dimension 42 .

LEMMA 10.1.1. The order of $G\left(H^{*}\right)$ is either 2, 6 or 14 . As an algebra $H$ is of one of the following types:

- $(1,2 ; 2,1 ; 6,1)$,

- $(1,2 ; 2,1 ; 3,4)$,

- $(1,2 ; 2,10)$,

- $(1,6 ; 6,1)$,

- $(1,6 ; 2,9)$,

- $(1,6 ; 3,4)$,

- $(1,14 ; 2,7)$.

Proof. Let $n=\left|G\left(H^{*}\right)\right|$. A counting argument, using 1.1, shows that $n \neq 7,21$, and the only possibilities are the prescribed ones if $n=6$ or 14 . If $n=1$ we find that $H$ must have an irreducible module of degree 2, which contradicts Corollary 2.2.3. If $n=3$, then $H$ is necessarily of type $(1,3 ; 2,3 ; 3,3)$, which is discarded by Theorem 2.2.1. 
Suppose finally that $n=2$. Then, either $H$ is of one of the prescribed algebra types, or else $H$ is of one of the following types:

$$
(1,2 ; 2,6 ; 4,1), \quad(1,2 ; 2,2 ; 4,2) .
$$

However, by Remark 2.2.2 (i), in these cases we have $G[\chi]=G\left(H^{*}\right)$, for all irreducible character $\chi$ of degree 2. By Theorem 2.4.2, $H$ has a Hopf algebra quotient $H \rightarrow \bar{H}$, where $\operatorname{dim} \bar{H}=2+6.4$ or $\operatorname{dim} \bar{H}=2+2.4$, respectively. By $[\mathbf{N Z}]$, this is not possible. Then the lemma follows.

REMARK 10.1.2. (i) Since 12 does not divide the dimension of $H$, by Theorem 2.2.1, every irreducible character of degree 2 is stable under left multiplication by some group-like element $g \in G\left(H^{*}\right)$ of order 2 .

(ii) It follows from Lemma 10.1.1 that $G[\chi]$ is nontrivial for all irreducible character $\chi$ of degree 6 . Also, if $\left|G\left(H^{*}\right)\right|=6$, then for all irreducible character $\chi$ of degree $3, G[\chi]$ is of order 3 .

(iii) It follows from Lemma 10.1.1 that $G\left(H^{*}\right)$ always contains a subgroup of order 2 .

Lemma 10.1.3. (i) Suppose $H$ is of type $(1,2 ; 2,10)$ as a coalgebra. Then $H$ is commutative.

(ii) Suppose $H$ is of type $(1,2 ; 2,1 ; 6,1)$ or $(1,2 ; 2,1 ; 3,4)$ as a coalgebra. Then $H$ contains a Hopf subalgebra isomorphic to $k^{\mathbb{S}_{3}}$, where $\mathbb{S}_{3}$ is the symmetric group on 3 symbols.

Proof. (i) This follows from Theorem 4.6.5, since by Remark 10.1.2 (iii), $\left|G\left(H^{*}\right)\right|$ is even.

(ii) In this case $H$ has a unique simple subcoalgebra $C$ of dimension 4, necessarily stable under left and right multiplication by $G(H)$. Therefore $A=k G(H) \oplus C$ is a Hopf subalgebra of $H$ of dimension 6 , which is not cocommutative; hence, in view of the classification of semisimple Hopf algebras of dimension $6, A \simeq k^{\mathbb{S}_{3}}$.

LEMma 10.1.4. Suppose that $H$ is of type $(1,14 ; 2,7)$ as a coalgebra. Then $H$ is commutative.

Proof. Note that the group $G\left(H^{*}\right)$ must be abelian by Proposition 1.2.6. Suppose that $G\left(H^{*}\right)$ is of order 2 or 6 . By Lemmas 10.1 .1 and 10.1.3, either there is a quotient Hopf algebra $p: H \rightarrow k G$, where $G$ is a group of order 6 , or else $G\left(H^{*}\right) \simeq \mathbb{S}_{3}$ is nonabelian of order 6. In the first case, a dimension argument implies that $H^{\operatorname{co} p}=k \Gamma$, where $\Gamma \subseteq G(H)$ is the only subgroup of order 7 . Therefore, $H$ fits into the (abelian) extension $1 \rightarrow k \Gamma \rightarrow H \rightarrow k G \rightarrow 1$, and the lemma follows from [N, Section 4]. In the second case, there is a projection $q: H \rightarrow k T \simeq k^{T}$, where $T \subseteq G\left(H^{*}\right)$ is the only subgroup of order 3; hence $\operatorname{dim} H^{\text {co } q}=14$, and $\Gamma \subseteq H^{\text {co } q}$. A dimension argument shows 
that $G(H) \subseteq H^{\operatorname{co} q}$ and thus $k G(H)=H^{\operatorname{co} q}$. Therefore $H$ fits into an abelian exact sequence $1 \rightarrow k G(H) \rightarrow H \rightarrow k T \rightarrow 1$, and the lemma follows also from $[\mathbf{N}$, Section 4$]$.

We claim that $H^{*}$ is not of type $(1,14 ; 2,7)$ as a coalgebra. Indeed, in this case, we have $G(H) \simeq G\left(H^{*}\right)$ is abelian and $H \simeq R \# k G(H)$ is a biproduct, where $R$ is of dimension 3. In particular, $R$ is commutative and cocommutative, and it follows easily that the only subgroup $F$ of order 7 of $H$ acts trivially on $R$. Then $F$ is central in $H$, and there is a central extension $0 \rightarrow k F \rightarrow H \rightarrow K \rightarrow 1$. Now, since every 6-dimensional semisimple Hopf algebra is trivial and since $\left|G\left(H^{*}\right)\right|$ is not divisible by 3 , we find that $K$ is necessarily isomorphic to a group algebra. In particular, this extension is abelian. This is a contradiction since $|G(H)| \neq 6$. This completes the proof of the lemma.

LEMMA 10.1.5. $H$ is not of type $(1,6 ; 6,1)$ as a coalgebra.

Proof. Suppose on the contrary that $H$ is of type $(1,6 ; 6,1)$ as a coalgebra. It follows from Lemmas 10.1.1, 10.1.3 and 10.1.4 that there is a quotient Hopf algebra $H \rightarrow A$, where $A$ is of dimension 6 . Hence, $A \simeq k G(H)$ and $H$ is a biproduct $H \simeq R \# k G$, where $R \simeq H / H(k G)^{+}$ is a braided Hopf algebra over $G$ of dimension 7 , and $G=G(H)$.

As a left coideal of $H, R$ decomposes as a direct sum $R=k 1 \oplus V$, where $V$ is an irreducible left coideal of dimension 6 . In particular, $V$ is a subcoalgebra of $R$ in the category of Yetter-Drinfeld modules over $G$, and the smash coproduct coalgebra $V \# k G$ coincides with the unique simple subcoalgebra of $H$ of dimension 36. Since $H^{2}\left(G, k^{\times}\right)=1$, it follows from $[\mathbf{N 2}, 1.3 .1]$ that $V$ is cocommutative and the action of $G$ permutes the 6 distinct group-like elements. Thus $V$ has a basis $x_{i}$, $1 \leq i \leq 6$, consisting of group-like elements of $R$.

By $[\mathbf{N 3}, 2.2], V x_{i}$ is a left coideal of $R$ containing 1 , and $V x_{i} \simeq V x_{j}$, for all $1 \leq i, j \leq 6$ such that $g x_{i}=x_{j}$, for some $g \in G$. Note that $x_{i}$ does not belong to $V x_{i}$ : indeed, since $x_{i}$ is invertible (with inverse $\left.x_{i}^{-1}=\mathcal{S}_{R}\left(x_{i}\right)\right)$, if $x_{i}=\sum_{j} x_{j} x_{i}$, then $1 \in \sum_{j} x_{j} \in V$, which is absurd.

Decomposing $V x_{i}$ into a direct sum of irreducible left coideals of $R$, we get $V x_{i}=k 1 \oplus \bigoplus_{l \neq i} k x_{l}$, where $g_{i} \neq g$, for all $i$. If $g \in G$ is such that $g x_{i}=x_{j} \neq x_{i}$, we have $g x_{i} \in V x_{i}$. But then $x_{j} \in V x_{j} \simeq V x_{i}$. This is a contradiction. Then the lemma follows.

Lemma 10.1.6. Suppose $H$ is of coalgebra type $(1,2 ; 2,1 ; 6,1)$ or $(1,2 ; 2,1 ; 3,4)$. Then $H$ is commutative.

Proof. We shall show that $H$ fits into an abelian extension, and hence is trivial by $[\mathbf{N}$, Section 4]. 
By Lemma 10.1.3, $H$ has a Hopf subalgebra $A$ isomorphic to $k^{\mathbb{S}_{3}}$. We claim that there is no Hopf algebra surjection $p: H \rightarrow k \mathbb{S}_{3}$. If this were the case, then $A \cap H^{\operatorname{co} p}=k 1$. Indeed, as a left coideal of $H, H^{\operatorname{co} p}$ decomposes as a direct sum or irreducible left coideals. Note that $G(A)=G(H)$ intersects trivially $H^{\text {co } p}$ by $[\mathbf{N Z}]$. If $A \cap H^{\text {co } p} \neq k 1$, then there is a 2-dimensional irreducible left coideal $U$ of $A$ such that $U \subseteq H^{\operatorname{co} p}$. Then $H^{\operatorname{co} p}=k 1 \oplus U \oplus W$, where $W$ is a left coideal of $H$ of dimension 4 , such that $W$ contains no one-dimensional left coideal of $H$. This is not possible and therefore $A \cap H^{\operatorname{co} p}=k 1$ as claimed. But then the restriction $p: k^{\mathbb{S}_{3}} \rightarrow k \mathbb{S}_{3}$ is an isomorphism, which is not possible. This establishes the claim. In particular, $\left|G\left(H^{*}\right)\right| \neq 2$.

We may then assume that $\left|G\left(H^{*}\right)\right|=6$. Hence, by Lemma 10.1.5, $H^{*}$ is of type $(1,6 ; 3,4)$ or $(1,6 ; 2,9)$ as a coalgebra. Dualizing the inclusion $A \subseteq H$, we get a Hopf algebra quotient $p: H^{*} \rightarrow k \mathbb{S}_{3}$. By $[\mathbf{N Z}], G\left(H^{*}\right) \cap H^{\operatorname{co} p}=k 1$; so that the restriction $p: k G\left(H^{*}\right) \rightarrow k \mathbb{S}_{3}$ is an isomorphism. In particular, $G\left(H^{*}\right)$ is non-abelian and $H^{*}$ is isomorphic to a biproduct $H^{*} \simeq R \# k G\left(H^{*}\right)$, where $\operatorname{dim} R=7$. Let $\Gamma \subseteq G\left(H^{*}\right)$ be the unique subgroup of order 3 .

Case I. $H^{*}$ is of type $(1,6 ; 3,4)$.

In this case, $R=k 1 \oplus W_{1} \oplus W_{2}$, where $W_{i}$ are irreducible left coideals of $H^{*}$ of dimension 3. Since $\Gamma=G[\chi]$ for all irreducible $\chi$ of degree 3 , then $g W_{i} \simeq W_{i} \simeq W_{i} g$, for all $g \in \Gamma, i=1,2$.

Claim 10.1.7. $\rho\left(W_{i}\right)$ is not contained in $k \Gamma \otimes W_{i}$.

ProOF. Suppose on the contrary that $\rho\left(W_{1}\right) \subseteq k \Gamma \otimes W_{1}$. Let $\widetilde{R}:=k\left[W_{1}\right]$; then $\widetilde{R}$ is a subalgebra and subcoalgebra of $R$ which is stable under the action of $\Gamma$ and $\rho(\widetilde{R}) \subseteq k \Gamma \otimes \widetilde{R}$. Therefore $\widetilde{A}=\widetilde{R} \# k \Gamma$ is a Hopf subalgebra of $H^{*}$, and $\operatorname{dim} \widetilde{A}=3 \operatorname{dim} \widetilde{R}>12$. Then $\operatorname{dim} \widetilde{A}=$ 21 , thus $\widetilde{A}$ is commutative and normal, and moreover the quotient $H^{*} / H^{*} \widetilde{A}^{+}$is cocommutative, since $k \Gamma \subseteq \widetilde{A}$. Hence, $G\left(H^{*}\right)$ is abelian, which is a contradiction. This proves the claim.

Fix $i=1,2$. We may assume that there exists $0 \neq w \in W_{i}$, such that $\rho(w)=a \otimes w$, where $a \in G\left(H^{*}\right)$ is an element of order 2. Let $1 \neq g \in \Gamma$, so that $0 \neq g . w \in W_{i}$ is homogeneous of degree $g a g^{-1} \neq a$. Hence $W_{i}^{\operatorname{co} G\left(H^{*}\right)}=0$, and $R^{\operatorname{co} G\left(H^{*}\right)}=k 1$.

Dualizing, $H=R^{*} \# A$ and the subalgebra $\left(R^{*}\right)^{A}$ of invariants in $R^{*}$ is one-dimensional, since $\left(R^{*}\right)^{A} \simeq \operatorname{Hom}^{G\left(H^{*}\right)}(R, k)$. By [AN2, Section 8], $\left(R^{*}\right)^{A}$ is isomorphic to the Hecke algebra of the pair $A, H$; that is, $\left(R^{*}\right)^{A} \simeq e_{A} H e_{A}$, where $e_{A} \in A$ is the normalized integral. By [AN2, Theorem 4.4] there is a bijection between irreducible representations of 
$\left(R^{*}\right)^{A}$ and irreducible representations of $H$ appearing in $\operatorname{Ind}_{A}^{H} \epsilon_{A}$ with positive multiplicity. This is a contradiction, hence the lemma follows.

Case II. $H^{*}$ is of type $(1,6 ; 2,9)$.

We shall use the action defined in (1.2.5).

Claim 10.1.8. The action $\Gamma \times \Gamma \times X_{2} \rightarrow X_{2}$, given by $(g, h) \cdot \chi=$ $g \chi h^{-1}$ is freely transitive.

ProOF. Let $\chi \in X_{2}$. It will be enough to show that the stabilizer $(\Gamma \times \Gamma)_{\chi}$ is trivial. Let $g, h \in \Gamma$ such that $g \chi h^{-1}=\chi$. Then $G[\chi]=$ $G\left[g \chi h^{-1}\right]=g G[\chi] g^{-1}$. This implies that $g=1$, because $G[\chi]$ is of order 2 and $G\left(H^{*}\right)$ is not abelian. Therefore $\chi h^{-1}=\chi$, and $h=1$. This proves the claim.

Observe that there exists $\chi \in X_{2}$ such that $\chi^{*}=\chi$, because $\left|X_{2}\right|$ is odd. Hence $\chi^{2}=1+a+\chi^{\prime}$, where $\left(\chi^{\prime}\right)^{*}=\chi^{\prime}$, and $G[\chi]=\{1, a\}=G\left[\chi^{\prime}\right]$. Note also that $\chi \neq \chi^{\prime}$, since $H$ has no quotient of dimension 6 which is not commutative. Write $\chi^{\prime}=g \chi h^{-1}, g, h \in \Gamma$. Hence $G[\chi]=$ $G\left[\chi^{\prime}\right]=g G[\chi] g^{-1}$, implying $g=1$. So $\chi^{\prime}=\chi h^{-1}$ and $h \neq 1$. Thus $\chi h^{-1}=\chi^{\prime}=\left(\chi^{\prime}\right)^{*}=h \chi^{*}=h \chi$; which implies that $h \chi h=\chi$. This contradicts Claim 10.1.8. The proof of the lemma is now complete.

\subsection{Classification}

We shall now prove Theorem 3 . In view of the previous results, we may assume that $H$ is of type $(1,6 ; 2,9)$ or $(1,6 ; 3,4)$ as a coalgebra. The proof of the theorem of will follow from the next two lemmas.

LEMMA 10.2.1. Suppose that $H$ is of type $(1,6 ; 2,9)$ as a coalgebra. Then $H$ is isomorphic to $\mathcal{A}_{7}(3,2)$.

Proof. By the above, we also have $\left|G\left(H^{*}\right)\right|=6$. Therefore the groups $G(H)$ and $G\left(H^{*}\right)$ are both abelian. This implies that $G(H)$ contains a unique subgroup $F$ of order 2. In particular, $H \simeq R \# k F$ is a biproduct over $F$. The subgroup $F$ necessarily stabilizes all 4dimensional simple subcoalgebras. Therefore the quotient coalgebra $H / H(k F)^{+} \simeq R$ is cocommutative. It follows from Proposition 4.6.1 that $H$ fits into an abelian extension. This implies the lemma.

Lemma 10.2.2. Suppose that $H$ is of type $(1,6 ; 3,4)$ as a coalgebra. Then $H$ is isomorphic to $\mathcal{A}_{7}(2,3)$.

Proof. Also here we have $H \simeq R \# k G$ is a biproduct, where $G$ is the unique subgroup of order 3 of $G(H)$. By Remark 10.1.2, $G$ stabilizes all simple subcoalgebras of $H$ of dimension 9 . Then $R$ is a cocommutative coalgebra and the lemma follows from Proposition 4.6.1. 


\section{CHAPTER 11}

\section{Dimension 48}

\subsection{First reduction}

Let $H$ be a nontrivial semisimple Hopf algebra of dimension 48 .

LEMma 11.1.1. The order of $G\left(H^{*}\right)$ is either $2,3,4,6,8,12,16$ or 24. As an algebra, $H$ is of one of the following types:

- $(1,2 ; 2,3 ; 3,2 ; 4,1)$,

- $(1,3 ; 3,1 ; 6,1)$,

- $(1,3 ; 2,9 ; 3,1)$,

- $(1,3 ; 3,5)$,

- $(1,4 ; 2,2 ; 3,4)$,

- $(1,4 ; 2,3 ; 4,2)$,

- $(1,4 ; 2,7 ; 4,1)$,

- $(1,4 ; 2,11)$,

- $(1,4 ; 2,2 ; 6,1)$,

- $(1,6 ; 2,6 ; 3,2)$,

- $(1,8 ; 2,2 ; 4,2)$,

- $(1,8 ; 2,10)$,

- $(1,8 ; 2,6 ; 4,1)$,

- $(1,12 ; 2,9)$,

- $(1,12 ; 3,4)$,

- $(1,12 ; 6,1)$,

- $(1,16 ; 2,8)$,

- $(1,16 ; 4,2)$,

- $(1,24 ; 2,6)$.

Proof. The possibility $\left|G\left(H^{*}\right)\right|=1$ is discarded by 1.1 and Theorem 2.2.1; see Corollary 2.2.3.

The only possibilities with $\left|G\left(H^{*}\right)\right|=2$ are the types $(1,2 ; 2,7 ; 3,2)$ and $(1,2 ; 2,3 ; 3,2 ; 4,1)$. In the first case, $H$ cannot have Hopf algebra quotients of type $(1,3 ; 3,1)$. Therefore, by Remark 2.2.2 (i) every irreducible character of degree 2 is stable under left multiplication by $G\left(H^{*}\right)$; hence by Theorem 2.4.2 there is a quotient Hopf algebra of dimension 30 , which is impossible. 
Suppose that $\left|G\left(H^{*}\right)\right|=16$. The only possibility excluded in the list is the type $(1,16 ; 2,4 ; 4,1)$. Suppose on the contrary that $H$ is of this type. Then $H$ has four irreducible characters of degree 2 and one irreducible character of degree 4. In particular, the action of $G\left(H^{*}\right)$ on $X_{2}$ is transitive and $|G[\chi]|=4$, for all irreducible character of degree 2. By Remark 2.4.3 (iii), there is a quotient Hopf algebra of dimension 32 , which is impossible. The rest of the lemma follows from 1.1.

REMARK 11.1.2. (i) If $H$ is of type $(1,24 ; 2,6)$, then $H$ is not simple, by Corollary 1.4.3.

(ii) Suppose that $H$ is of type $(1,4 ; 2,3 ; 4,2)$ as a coalgebra. By Theorem 2.4.2 the irreducible characters of degrees 1 and 2 give rise to a Hopf subalgebra of dimension 16 .

(iii) If $H$ is of type $(1,8 ; 2,2 ; 4,2)$ as a coalgebra then the irreducible characters of degrees 1 and 2 give a Hopf subalgebra of dimension 16; see Remark 2.4.3 (iii).

(iv) Suppose that $H$ is of type $(1,2 ; 2,3 ; 3,2 ; 4,1)$ as a coalgebra. Then $H$ is not simple.

Proof. We have that $H$ does not contain Hopf subalgebras of dimension $12[\mathbf{F}]$. On the other hand, there must exist an irreducible character $\chi$ of degree 2, such that $G[\chi]=1$ (since otherwise there would be a Hopf subalgebra of dimension 14). By Theorem 2.2.1, $H$ has a Hopf subalgebra of dimension 24 and then $H$ is not simple.

(v) Suppose that $H$ is of type $(1,4 ; 2,2 ; 3,4)$ as a coalgebra. Then $H$ contains unique Hopf subalgebras $A_{1} \subseteq A$ of dimension 6 and 12 , respectively.

Proof. The irreducible characters of degrees 1 and 2 give rise to a (unique) Hopf subalgebra of dimension 12.

Let $\psi_{1}, \ldots, \psi_{4}$ be the irreducible characters of degree 3 , and let $\chi_{1}, \chi_{2}$ be the irreducible characters of degree 2 . We have $\psi_{1}^{*} \psi_{1}=1+$ $\chi_{1}+\psi+\psi^{\prime}$, where $\psi, \psi^{\prime}$ are irreducible of degree 3 ; this implies that $\chi_{i}^{*}=\chi_{i}, i=1,2$. Thus $\psi_{1} \chi_{1}=\psi_{1}+\psi_{1} a$, where $1 \neq a \in G\left[\chi_{1}\right]$. In particular, $\left|G\left[\chi_{1}\right]\right|=\left|G\left[\chi_{2}\right]\right|=2$.

Let $\psi_{i}$ be any irreducible character of degree 3 ; thus $\psi_{i}=g \psi_{1}$, for some $g \in G(H)$. Hence $\psi_{i}^{*} \psi_{i}=\psi_{1}^{*} \psi_{1}=1+\chi_{1}+\psi+\psi^{\prime}$.

Then $\chi_{1}^{2}=1+a+\chi$, where $\chi$ is irreducible of degree 2. Comparing decompositions of $\psi_{1} \chi_{1}^{2}$, we find that $\psi_{1} \chi=\psi_{1}+\psi_{1} a$, implying that $m\left(\chi, \psi_{1}^{*} \psi_{1}\right)=1$ and in turn that $\chi=\chi_{1}$. Then $G\left[\chi_{1}\right] \cup\left\{\chi_{1}\right\}$ give rise to a Hopf subalgebra $A_{1}$ of dimension 6 . We have also $m\left(\chi_{1}, \chi_{2} \chi_{1}\right)=$ $m\left(\chi_{2}, \chi_{1}^{2}\right)=0$. Therefore $m\left(\chi_{2}, \chi_{2} \chi_{1}\right)=m\left(\chi_{2}, \chi_{1} \chi_{2}\right)=m\left(\chi_{1}, \chi_{2}^{2}\right)>0$, implying that $m\left(\chi_{2}, \chi_{2} \chi_{1}\right)=m\left(\chi_{1}, \chi_{2}^{2}\right)=1$. Thus $\chi_{2}=b \chi_{1}, b \neq a \in$ 
$G(H)$ and $\chi_{2}^{2}=1+a+\chi_{1}$. In particular, $A_{1}$ is the only six-dimensional Hopf subalgebra of $H$ as claimed.

Note that the set $\left\{\psi, \psi^{\prime}\right\}$ is stable under the adjoint action of $G(H)$ and also under $*$. Also, if $\psi=\psi^{\prime}$, then $H$ is not simple; indeed, in this case, $G\left[\chi_{1}\right], \chi_{1}, \psi$ and $a \psi=\psi a$ span a standard subalgebra corresponding to a Hopf subalgebra of dimension 24.

(vi) Suppose that $H$ is of type $(1,4 ; 2,2 ; 6,1)$ as a coalgebra. Then $H$ contains a unique Hopf subalgebra $A$ of dimension 12 , which coincides with the sum of simple subcoalgebras of dimension 1 and 4 .

LEMma 11.1.3. Suppose that $H$ is of type $(1,4 ; 2,7 ; 4,1)$ as a coalgebra. Then $H$ is not simple.

Proof. By Proposition 2.1.3, $H$ contains a Hopf subalgebra $A$ of dimension 8. In particular, the group $G(H)=G(A)$ is not cyclic.

Let $\zeta \in H$ be the unique irreducible character of degree 4 . Hence we have $g \zeta=\zeta=\zeta g$, for all $g \in G(H)$. Let $\lambda \in A$ be the unique irreducible character of degree 2 . We claim that

$$
\lambda \zeta=2 \zeta=\zeta \lambda \text {. }
$$

Indeed, otherwise, there would exist $\chi \in X_{2}$ such that $m(\chi, \lambda \zeta)>0$; hence $\lambda \zeta=\chi+\ldots$ Multiplying on the left by $\lambda$, and using that $\lambda^{2}=\sum_{g \in G(H)} g$, we find that $\lambda \chi=\zeta$ is irreducible. This contradicts Lemma 2.4.1. Thus $\lambda \zeta=2 \zeta$, and then $\zeta \lambda=(\lambda \zeta)^{*}=2 \zeta^{*}=2 \zeta$. Hence the claim is established.

This implies that $A C=C=C A$. It follows from Corollary 3.2.5 and [M5, Theorem $4.8(1)]$ that $A$ is commutative. Suppose that $B \subseteq$ $H$ is another Hopf subalgebra of dimension 8 . Then the same argument applies, and thus $k[A, B] C=C=C k[A, B]$. Hence $\operatorname{dim} k[A, B]=16$ and the Hopf subalgebra $k[A, B]$ is normal in $k[C]=H$. Therefore $H$ is not simple in this case.

Suppose next that $B \subseteq H$ is a Hopf subalgebra of dimension 6; we may assume that $k[A, B]=H$. We have $G(B) \cap Z(B) \neq 1$ and, since $A$ is commutative, this group is central in $k[A, B]=H$. Hence $H$ is not simple in this case.

Since $H$ cannot contain Hopf subalgebras of dimension 32, there exist $\chi, \psi \in X_{2}$ such that $\chi^{*} \psi=\zeta$ is irreducible of degree 4. It follows from Lemma 2.4.1 that $G[\chi]$ and $G[\psi]$ are distinct subgroups of order 2 of $G(H)$. Write $\psi \psi^{*}=1+a+\tau$, where $\tau \in X_{2}$ and $G[\psi]=\{1, a\}$; thus $\tau=\tau^{*}$ and $G[\psi] \subseteq G[\tau]$. Similarly, $\chi \chi^{*}=1+b+\mu$, where $\mu \in X_{2}$ and $G[\chi]=\{1, b\}$; thus $\mu=\mu^{*}$ and $G[\chi] \subseteq G[\mu]$. In view of Lemma 2.4.1, we have $\mu \neq \tau$. Hence, after changing if necessary $\psi$ and $\chi$, we may assume $\tau \neq \lambda$. 
Suppose $G[\tau]=G(H)$. Then $\tau$ and $G(H)$ span a standard subalgebra corresponding to a Hopf subalgebra of dimension 8, and we know $H$ is not be simple in this case. Hence we may assume $|G[\tau]|=2$. If $\tau=\psi g, g \in G(H)$, then $\tau^{2}=\psi g(\psi g)^{*}=1+a+\tau$. Thus $\tau$ and $G[\tau]$ span a standard subalgebra corresponding to a Hopf subalgebra of dimension 6 , and $H$ is not simple.

Therefore we may assume that the orbits $\tau G(H)$ and $\psi G(H)$ are disjoint. Then the orbits of the right action of $G(H)$ on $X_{2}$ are

$$
\lambda G(H), \quad \chi G(H), \quad \psi G(H), \quad \tau G(H),
$$

and the only orbits with (left) stabilizer $G[\psi]$ are $\psi G(H)$ and $\tau G(H)$.

Note that if $\pi \in X_{2}$ is such that $m(\pi, \psi \zeta)>0$, then $m\left(\zeta, \pi^{*} \psi\right)=$ $m(\pi, \psi \zeta)>0$. Therefore $G[\pi] \cap G[\psi]=1$ and $\pi \in \chi G(H)$. Thus $\psi \zeta=$ $\chi+\chi t+\zeta$, for some $t \in G(H)$. In particular, $m\left(\zeta, \zeta \psi^{*}\right)=m(\zeta, \psi \zeta)=1$.

On the other hand, $\zeta \psi^{*}=\chi^{*} \psi \psi^{*}=\chi^{*}+\chi^{*} a+\chi^{*} \tau$. Hence $\chi^{*} \tau=\zeta$ is irreducible. Letting $X=\left\{\rho \in X_{2}: \chi^{*} \rho=\zeta\right\}$, we have $X=$ $\psi G(H) \cup \tau G(H)$. Moreover, we have $G(H) X=X=X G(H)$ : the left hand side equality, because $G[g \rho]=G[\rho]$, for all $g \in G(H)$, and the only elements in $X_{2}$ with stabilizer $G[\psi]$ are in $\psi G(H) \cup \tau G(H)=X$.

Let $\sigma, \rho \in X^{\prime}=X \cup\{\lambda\}$. Then the product $\sigma^{*} \rho \neq \zeta$. Note also that, for all $\rho \in X$, we have $m(\chi, \rho \zeta)=m(\chi g, \rho \zeta)=m\left(\zeta, \chi^{*} \rho\right)=1$. Hence we have $\rho \zeta=\chi+\chi t+\zeta$, and thus $m\left(\rho, \zeta^{2}\right)=1$, for all $\rho \in X$.

This allows us to write $\zeta^{2}=\sum_{g \in G(H)} g+2 \lambda+\sum_{\rho \in X} \rho$. In particular, it follows that $X^{\prime}$ is closed under $*$. If $\sigma \in X$, then

$$
\begin{aligned}
\sigma \zeta^{2} & =\sum_{g \in G(H)} \sigma g+2 \sigma \lambda+\sum_{\rho \in X} \sigma \rho \\
& =(\sigma \zeta) \zeta=(\chi+\chi t+\zeta) \zeta=2 \chi \zeta+\zeta^{2} \\
& =2 \sum_{\nu \in X} \nu+\sum_{g \in G(H)} g+2 \lambda+\sum_{\rho \in X} \rho .
\end{aligned}
$$

This implies that for all $\sigma, \rho \in X$, the product $\rho \sigma$ decomposes as a sum of elements of $X^{\prime} \cup G(H)$. Also, since $\lambda \zeta^{2}=2 \zeta^{2}=\zeta^{2} \lambda$, then all products $\lambda \sigma, \sigma \lambda$ decompose as sums of elements of $X$, for all $\sigma \in X$.

Hence $X^{\prime} \cup G(H)$ spans a standard subalgebra of $R\left(H^{*}\right)$ which corresponds to a Hopf subalgebra of dimension 24. This proves the lemma.

Lemma 11.1.4. Assume that $H$ is of type $(1,6 ; 2,6 ; 3,2)$ as a coalgebra. Then $H$ is not simple. 
Proof. Since the dimension of $H$ is not divisible by 30, there must exist $\chi \in X_{2}$ such that $G[\chi]=1$. By Theorem 2.2.1, $H$ has a Hopf subalgebra $A$ of type $(1,3 ; 3,1)$ as a coalgebra.

Let $\psi \in \widehat{H^{*}}$ be the unique irreducible character of degree 3 in $A$. Then we have $\psi^{2}=1+a+a^{2}+2 \psi$, where $G[\psi]=\left\{1, a, a^{2}\right\}$. Let $b \in G(H)$ of order 2 , so that $b \psi=\psi^{\prime}=\psi b$ is the remaining degree 3 irreducible character. We have $\psi^{\prime} \psi, \psi \psi^{\prime}$ belong to the span of $G(H) \cup\left\{\psi, \psi^{\prime}\right\}$. Thus $G(H) \cup\left\{\psi, \psi^{\prime}\right\}$ spans a standard subalgebra of $H$ which corresponds to a Hopf subalgebra of dimension 24. This implies that $H$ is not simple, as claimed.

Lemma 11.1.5. Assume that $H$ is of type $(1,3 ; 3,5)$ as a coalgebra. Then $H$ is not simple.

Proof. Let $\psi \in H$ be an irreducible character of degree 3. Decomposing the product $\psi \psi^{*}$, we see that $G[\psi]=G(H)$ is necessarily of order 3 .

By Remark 3.2.7, the quotient coalgebra $H / H(k G(H))^{+}$is cocommutative. In particular, we may assume that $\left|G\left(H^{*}\right)\right|$ is not divisible by 3 : otherwise $H$ would be a biproduct $H=R \# k G(H)$, with $R \simeq H / H(k G(H))^{+}$cocommutative, implying that $H$ is not simple, by Proposition 4.6.1.

By Lemma 11.1.1 and the previous lemmas, we may assume that there is a Hopf algebra quotient $H \rightarrow B$, where $\operatorname{dim} B=4$; hence $\operatorname{dim} H^{\operatorname{co} B}=12$, and by $[\mathbf{N Z}], k G(H) \subseteq H^{\operatorname{co} B}$.

Let $H^{\text {co } B}=k G(H) \oplus V_{1} \oplus V_{2} \oplus V_{3}$ be a decomposition of $H^{\text {co } B}$ as a sum of irreducible left coideals of $H$. If $V_{1}, V_{2}$ and $V_{3}$ are pairwise isomorphic, then $H^{\mathrm{co} B}$ is a subcoalgebra of $H$ and the map $H \rightarrow B$ is normal. Hence we may assume that $V_{1}$ appears with multiplicity 1 in $H^{\operatorname{co} B}$.

Suppose that $V_{i}$ appears with multiplicity 1 in $H^{\text {co } B}$. Then necessarily $g V_{i}=V_{i}=V_{i} g$, for all $g \in G(H), i=1,2,3$. Let $C_{i} \subseteq H$ be the simple subcoalgebra containing $V_{i}$. By Corollary 3.5.2 $k G(H)$ is normal in $k\left[C_{i}\right]$. Hence, we may assume that $\operatorname{dim} k\left[C_{i}\right]=12$.

We claim that $V_{i}$ appears with multiplicity 1, for all $i$. As above, implies that $G(H)$ is normal in $k\left[C_{i}\right]$ for all $i$. Since $k\left[C_{1}, C_{2}, C_{3}\right]=H$, it follows that $G(H)$ is normal in $H$ and we are done.

To prove the claim we argue as follows. By Lemma 1.7.1, $\left(H^{\operatorname{co} B}\right)^{*} \simeq$ $\operatorname{Ind}_{B^{*}}^{H^{*}} 1$ as left $H^{*}$-modules. Hence, $\operatorname{Ind}_{B^{*}}^{H^{*}} 1=\sum_{g \in G(H)} g+\psi_{1}+2 \psi_{2}$, where $\psi_{1} \neq \psi_{2} \in H$ are irreducible characters of degree 3 . In particular, $\psi_{2}^{*}=\psi_{2}$.

We may also assume that the Hopf subalgebra $k[C]$, where $C$ is the subcoalgebra corresponding to $\psi_{2}$, is all of $H$; otherwise, $\operatorname{dim} k[C]=12$ 
and $G(H)$ would be normal in $k[C]$, hence $G(H)$ would be normal in $H=k\left[C, C_{1}\right]$ (by dimension).

By Frobenius reciprocity, $\left.\psi_{2}\right|_{B^{*}}=2.1+t$, where $1 \neq t \in G(B)$. Since $\psi_{2}^{*}=\psi_{2}$, then $t^{2}=1$. Let $A=k\langle t\rangle \subseteq B$. Consider the composition

$$
\pi: H \rightarrow B \rightarrow \bar{B}:=B / B A^{+} .
$$

We have $\left.\psi_{2}\right|_{B}=\pi\left(\psi_{2}\right)=3$.1. Applying again the Frobenius reciprocity, this gives $m\left(\psi_{2}, \operatorname{Ind} \bar{H}^{*}{ }^{*} 1\right)=3=\operatorname{deg} \psi_{2}$.

Therefore, by Lemma 1.7.1, $H^{\text {co } \bar{B}}$ contains the simple subcoalgebra $C$ corresponding to $\psi_{2}$. This is absurd, since it implies that $k[C]=$ $H \subseteq H^{\text {co } \bar{B}}$. This finishes the proof of the lemma.

Lemma 11.1.6. Assume that $H$ is of type $(1,3 ; 2,9 ; 3,1)$ as a coalgebra. Then $H$ is not simple.

ProOf. Let $\psi$ be the unique irreducible character of degree 3. For all irreducible character $\mu$ of degree 2, we have $\mu \mu^{*}=1+\psi$. By Theorem 2.2.1, $G(H)$ and $\psi$ span a standard subalgebra of $R(H)$, which corresponds to a commutative Hopf subalgebra of coalgebra type $(1,3 ; 3,1)$. In particular, the dimension of an irreducible $H$-module is at most $[H: A]=4$. Thus $H^{*}$ cannot be of type $(1,3 ; 3,1 ; 6,1)$. Moreover, by previous results in this section, we may assume that either $H^{*}$ is of type $(1,3 ; 2,9 ; 3,1)$ as a coalgebra, or else there is a Hopf algebra quotient $H \rightarrow B$, where $\operatorname{dim} B=4$.

In the last case, we have $\operatorname{dim} H^{\operatorname{co} B}=12$ and $G(H) \subseteq H^{\operatorname{co} B}$, by [NZ]. Also, unless $A=H^{\text {co } B}$ is normal in $H$, we may assume that $H^{\text {co } B}$ contains an irreducible left coideal $V$ of dimension 2. Therefore, $H^{\text {co } B}=k G(H) \oplus \oplus_{g \in G(H)} g V \oplus W$ as a left coideal of $H$, where $W$ is an irreducible left coideal of dimension 3. This implies that $G(H) \chi=$ $\chi G(H)$, where $\chi$ is the character of $V$. Moreover, by Lemma 1.7.1, there exists $g_{0} \in G(H)$ such that $\chi^{*}=g_{0} \chi$.

The relation $\chi^{*} \chi=1+\psi$ implies that $\psi \chi=\sum_{g \in G(H)} g \chi$. Also, for all $g, h \in G(H)$, we have $\chi h=h_{0} \chi$, for some $h_{0} \in G(H)$ and therefore,

$$
(g \chi)(h \chi)=g h_{0} \chi^{2}=g h_{0}\left(g_{0}\right)^{-1} \chi^{*} \chi,
$$

so that $(g \chi)(h \chi)$ belongs to the span of $G(H)$ and $\psi$. It follows that $G(H), \psi$ and $G(H) \chi$ span a standard subalgebra, corresponding to a Hopf subalgebra of dimension 24. Hence $H$ is not simple in this case.

Suppose finally that $H^{*}$ is of type $(1,3 ; 2,9 ; 3,1)$ as a coalgebra. Then there is a projection $q: H \rightarrow B$, where $B=k G(B)$ is of dimension 12. The coalgebra structure of $B^{*}$ implies that $G(B)$ has a unique normal subgroup of order 4 . We have necessarily $H^{\text {co } B}=k 1 \oplus V$, where 
$V$ is an irreducible left coideal of dimension 3; whence $\operatorname{dim} q(A)=3$ and $q(\psi)=\sum_{x \in G(q(A))} x$.

Since $\left|X_{2}\right|$ is odd, there exists an irreducible character $\chi$ of degree 2 , such that $\chi^{*}=\chi$. Then $q(\chi)=a+b$, where $a, b \in G(B)$. The relation $\chi^{2}=1+\psi$ implies that $G(q(A)) \subseteq\langle a, b\rangle$. We cannot have $a^{2}=b^{2}=1$, since otherwise $\langle a, b\rangle$ would be contained in the unique subgroup of order 4 of $G(B)$. Hence $b=a^{-1}$, and $G(q(A))=\langle a\rangle$ of order 3 .

Let $C$ be the simple subcoalgebra containing $\chi$. Then $q(k[C])=\langle a\rangle$; in particular, $k[C] \neq H$. In addition, $A \subseteq k[C]$, so that $12 / \operatorname{dim} k[C]$. Hence, $\operatorname{dim} k[C]=24$ and thus $H$ is not simple. This finishes the proof of the lemma.

LEMMA 11.1.7. Let $H$ be of type $(1,8 ; 2,2 ; 4,2)$ or $(1,8 ; 2,10)$ as a coalgebra. Assume $H$ is simple. Then $H$ contains a Hopf subalgebra of dimension 16.

Proof. If $H$ is of type $(1,8 ; 2,2 ; 4,2)$, the claim follows from Remark 11.1.2 (iii). So assume $H$ is of type $(1,8 ; 2,10)$. For all $\lambda \in X_{2}$ we have $|G[\lambda]|=2$ or 4 .

Suppose $\chi \in X_{2}$ is such that $G[\chi]=G\left[\chi^{*}\right]$ of order 4 . Let $A=$ $k G[\chi]$ and $C$ the simple subcoalgebra containing $\chi$. We have $A C=$ $C=C A$, whence $A$ is normal in $k[C]$ by Proposition 3.2.6. Since $|G[\chi]|=4, A$ is normal in $k G(H)$ and then it is also normal in $K=$ $k[C, G(H)]$. But $\operatorname{dim} K>8$ is divisible by 8 , and we are assuming that $H$ is simple. Hence $\operatorname{dim} K=16$ and we are done.

Assume first that $|G[\lambda]|=4$ for all $\lambda \in X_{2}$. We claim that there exists $\chi \in X_{2}$ such that $G[\chi]=G\left[\chi^{*}\right]$, implying the lemma. To prove the claim, consider the action of $G(H) \times G(H)$ on $X_{2}$ given by $(g, h) \cdot \chi=$ $g \chi h^{-1}$. The orbit of an element $\chi$ is $G(H) \chi G(H)$, so it has order 2 or 4, and clearly $G(H) \chi G(H)$ and $G(H) \chi^{*} G(H)$ are of the same order, for all $\chi$. Also, because $G[\chi]$ is normal in $G(H), G[\lambda]=G[\chi]$ for all $\lambda \in G(H) \chi G(H)$. Suppose on the contrary that $G[\chi] \neq G\left[\chi^{*}\right]$ for all $\chi \in X_{2}$. Then $G(H) \chi G(H)$ and $G(H) \chi^{*} G(H)$ are disjoint. Let $\alpha \in X_{2}$ such that $\alpha$ is not conjugate to $\chi$ nor to $\chi^{*}$ (such an $\alpha$ exists because $\left.\left|X_{2}\right|=10\right)$. Then the orbits $G(H) \chi G(H), G(H) \chi^{*} G(H), G(H) \alpha G(H)$ and $G(H) \alpha^{*} G(H)$ are pairwise disjoint.

Then $\left|X_{2}\right|=10 \geq 2|G(H) \chi G(H)|+2|G(H) \alpha G(H)| \geq 8$, and there must exist $\beta$ not in any of these orbits. Hence, by dimension, $G(H) \beta G(H)=G(H) \beta^{*} G(H)$. Thus $G[\beta]=G\left[\beta^{*}\right]$ and the lemma holds in this case.

Suppose next that $|G[\lambda]|=2$ for some $\lambda \in X_{2}$. Then $\lambda \lambda^{*}=$ $\sum_{g \in G[\lambda]} g+\chi$ for some $\chi \in X_{2}$ such that $\chi^{*}=\chi$. If $|G[\chi]|=4$, then we 
are done. Otherwise, we may assume $\chi G(H)$ is the only orbit with 4 elements: if not, since $\left|X_{2}\right|=10$, there would be a unique orbit $\alpha G(H)$ with stabilizer of order 4 , and thus $G\left[\alpha^{*}\right]=G[\alpha]$ implying the lemma.

In particular, for all $g \in G(H), g \chi g^{-1}=\chi a, a \in G(H)$, and therefore $G\left[g \chi g^{-1}\right]=g G[\chi] g^{-1}=G[\chi]$. That is, $G[\chi] \unlhd G(H)$.

Also $\lambda=\chi t, t \in G(H)$, and $\chi^{2}=\sum_{g \in G[\chi]} g+\chi$. Hence $G[\chi]$ and $\chi$ span a standard subalgebra corresponding to a Hopf subalgebra $A$ of dimension 6. Moreover, $G[\chi]$ is central in $A$. Then $k G[\chi]$ is normal in $k[A, G(H)]$. But this implies $\operatorname{dim} k[A, G(H)]=24$ or 48 , and $H$ is not simple in this case.

LEMma 11.1.8. Suppose that $H$ is of type $(1,8 ; 2,6 ; 4,1)$ as a coalgebra. Then $H$ is not simple.

Proof. Let $\zeta \in C$ be the unique irreducible character of degree 4 in $H$. Let $X=\left\{\chi \in X_{2}: m\left(\chi, \zeta^{2}\right)>0\right\}$, so that $\zeta^{2}=\sum_{g \in G(H)} g+$ $\sum_{\chi \in X} n_{\chi} \chi+n \zeta$, where $n_{\chi}=m\left(\chi, \zeta^{2}\right)=m(\zeta, \chi \zeta)=1$ or $2, \chi \in X$. We have $X$ is stable under $*$ and left and right multiplication by $G(H)$.

If $X=\emptyset$ then $G(H)$ and $\zeta$ span a standard subalgebra corresponding to a Hopf subalgebra of dimension 24. Hence $H$ is not simple in this case. Thus we may assume $X \neq \emptyset$.

Let $\chi \in X$. We have $\chi \zeta=n_{\chi} \zeta+\sum \lambda$, where $\lambda$ runs over the set $\Lambda=\left\{\lambda \in X_{2}: m(\lambda, \chi \zeta)=m\left(\zeta, \lambda^{*} \chi\right)>0\right\}$. Then $\operatorname{deg} \chi \zeta=4 n_{\chi}+2|\Lambda|$. Note that $\Lambda G(H)=\Lambda$, so that $|\Lambda|=0$ (iff $n_{\chi}=2$ ) or 2 (iff $n_{\chi}=1$ ).

If $|\Lambda|=0$, we have $n_{\chi}=2$. Since $g \chi$ appears in $\zeta^{2}$ with the same multiplicity as $\chi$, for all $g \in G(H)$. Then $|G[\chi]|=4$ and $\zeta^{2}=$ $\sum_{g \in G(H)} g+2 \chi+2 t \chi$, for some $t \in G(H)$. In particular $G(H) \chi=$ $\chi G(H)$. Also $\chi \zeta^{2}=2 \zeta^{2}$. Then $G(H), G(H) \chi$ and $\zeta$ span a standard subalgebra corresponding to a Hopf subalgebra of dimension 32. This is impossible by $[\mathbf{N Z}]$. Hence $|\Lambda|=2$; say $\Lambda=\{\lambda, \lambda a\}, a \in G(H)$. In particular, $\left|G\left[\lambda^{*}\right]\right|=|G[\lambda]|=4$. Then $n_{\chi}=1$, for all $\chi \in X$. So that $\zeta^{2}=\sum_{g \in G(H)} g+\chi_{1}+\chi_{2}+\zeta$, with $X=\left\{\chi_{1}, \chi_{2}\right\}$, if $|X|=2$, or $\zeta^{2}=\sum_{g \in G(H)} g+\chi_{1}+\chi_{2}+\chi_{3}+\chi_{4}$, with $X=\left\{\chi_{1}, \chi_{2}, \chi_{3}, \chi_{4}\right\}$, if $|X|=4$.

Then, for each $\chi \in X, \chi \zeta=\zeta+\lambda+\lambda a$, and thus $\chi \zeta^{2}=\zeta^{2}+$ $\lambda(1+a) \zeta=\zeta^{2}+2 \lambda \zeta$. Now since $\lambda^{*} \chi=\zeta$, then $\lambda \zeta=\lambda \lambda^{*} \chi \in G(H) \chi$. Hence $\chi \zeta^{2}$ belongs to the span of $G(H), \zeta$ and $X$. Since this happens for all $\chi \in X$, then the irreducible summands of $\zeta^{2}$ span a standard subalgebra corresponding to a Hopf subalgebra of dimension 32 or 24 . By $[\mathbf{N Z}]$ the first case is impossible, and the second one implies that $H$ is not simple. 
Lemma 11.1.9. Suppose $H$ is of type $(1,3 ; 3,1 ; 6,1)$ as a coalgebra. Then $H$ is not simple.

ProOF. The irreducible characters of degrees 1 and 3 span a standard subalgebra of $R(H)$, which corresponds to a Hopf subalgebra $A$ of dimension 12. By $[\mathbf{F}] A$ is commutative. In particular, $\operatorname{dim} V \leq[H$ : $A]=4$, for all irreducible $H$-module $V$.

Suppose on the contrary that $H$ is simple. Then there is no Hopf algebra quotient $H \rightarrow B$ with $\operatorname{dim} B=16$ : otherwise, by $[\mathbf{N Z}]$, $k G(H)=H^{\text {co } B}$ is a normal Hopf subalgebra of $H$. In view of Lemma 11.1.1 and the previous results, it follows that $\operatorname{dim} V=1,2$ or 3 for all irreducible $H$-module $V$. It follows as well that $\left|G\left(H^{*}\right)\right|=4$ or 12 , and thus $G\left(H^{*}\right)$ contains a subgroup $\Gamma$ of order 4 .

Consider the Hopf algebra projection $H^{*} \rightarrow A^{*}$. Then $\left(H^{*}\right)^{\text {co } A^{*}} \subseteq$ $\left(H^{*}\right)^{\operatorname{co} q}$, where $q: H^{*} \rightarrow k^{G(H)}$ is the natural projection. By [NZ], we have $k \Gamma \subseteq\left(H^{*}\right)^{\text {co } q}$. In particular, since $\left(H^{*}\right)^{\operatorname{co} q} \neq k G\left(H^{*}\right), \Gamma=$ $G\left(H^{*}\right) \cap\left(H^{*}\right)^{\text {co } q}$ is a normal subgroup of $G\left(H^{*}\right)$, and it is therefore its only subgroup of order 4 .

Claim 11.1.10. $\left(H^{*}\right)^{\operatorname{co} q}$ contains no irreducible left coideal of dimension 3.

Proof. Suppose otherwise that $U \subseteq\left(H^{*}\right)^{\operatorname{co} q}$ is an irreducible left coideal of dimension 3 . Hence $H^{*}$ is of type $(1,4 ; 2,2 ; 3,4)$ or $(1,12 ; 3,4)$ as a coalgebra, and there is a Hopf subalgebra $B \subseteq H^{*}$ with $\operatorname{dim} B=$ 12 , such that $B$ is cocommutative or of type $(1,4 ; 2,2)$.

Since $g U$ is not isomorphic to $U$, for all $g \in \Gamma$, the sum $\sum_{g \in \Gamma} g U$ is direct and by dimension, $\left(H^{*}\right)^{\operatorname{co} q}=k \Gamma \oplus \oplus_{g \in \Gamma} g U$. Hence, if $H$ is simple, $\left(H^{*}\right)^{\text {co } A^{*}}=k 1 \oplus g U$, for some $g \in \Gamma$. This implies that $B^{\text {co } A^{*}}=$ $B \cap\left(H^{*}\right)^{\text {co } A^{*}}=k 1$, and therefore that $B \simeq A^{*}$ is cocommutative and $H^{*} \simeq R \# B$ is a biproduct, where $R$ is a braided Hopf algebra over $B$ of dimension 4. Proposition 4.4.6 implies that $H$ is not simple in this case. Hence the claim follows.

In view of the claim, we may assume that $\left(H^{*}\right)^{\text {co } A^{*}}=k 1 \oplus k t \oplus V$, where $1 \neq t \in G\left(H^{*}\right)$, and $V$ is an irreducible left coideal of dimension 2. In particular, $t V=V=V t$. So that, letting $C$ be the simple subcoalgebra of $H^{*}$ containing $V$, we find that $t$ is central in the Hopf subalgebra $k[C]$, by Corollary 3.5.2. In particular, $k[C] \neq H^{*}$ and by $[\mathbf{N Z}]$, $\operatorname{dim} k[C]=12$ or 8 . In the first case, the restriction $q \mid: k[C] \rightarrow k^{G(H)}$ is surjective; then by $[\mathbf{F}] k[C]$ is trivial, whence necessarily commutative. But this implies that $\operatorname{dim} U \leq\left[H^{*}: k[C]\right]=4$, for all irreducible $H$-comodules $U$, against the assumption on the coalgebra type of $H$. Hence $\operatorname{dim} k[C]=8$ and $k[C] \subseteq\left(H^{*}\right)^{\operatorname{co} q}$. 
We have therefore a decomposition $\left(H^{*}\right)^{\operatorname{co} q}=k[C] \oplus V_{1} \oplus V_{2} \oplus V_{3} \oplus V_{4}$, where $V_{i}$ is an irreducible left coideal of dimension 2 of $H^{*}$, for all $i=1, \ldots, 4$.

Note that $V_{i}$ appears in $\left(H^{*}\right)^{\operatorname{co} q}$ with multiplicity 1 , for all $i$. Otherwise, say $C_{1} \subseteq\left(H^{*}\right)^{\text {co } q}$, where $C_{1}$ is the simple subcoalgebra containing $V_{1}$. Then $k\left[C, C_{1}\right] \subseteq\left(H^{*}\right)^{\operatorname{co} q}$; but the inclusion $k[C] \subseteq k\left[C, C_{1}\right]$ is strict, so $k\left[C, C_{1}\right]=\left(H^{*}\right)^{\mathrm{co} q}$ by dimension restrictions, implying that $H$ is not simple.

Let $\tau_{i} \in X_{2}$ be the irreducible character corresponding to $V_{i}, i=$ $1, \ldots, 4$, and let $\lambda$ be the character of $V$. By Frobenius reciprocity $m\left(1, q\left(\tau_{i} \tau_{i}^{*}\right)\right)=2$, thus $G\left[\tau_{i}\right] \neq \Gamma$, because $q(g)=1$, for all $g \in \Gamma$. Hence $G\left[\tau_{i}\right]$ is a subgroup of order 2 , for all $i=1, \ldots, 4$. Similarly, we see that $m\left(\tau_{i}, \lambda \tau_{i}\right)=m\left(\lambda, \tau_{i} \tau_{i}^{*}\right)=0$

Without loss of generality we may write $\tau_{2}=h \tau_{1}$ and $\tau_{4}=g \tau_{3}$, for some $h, g \in \Gamma \backslash\{1\}$. By the above, $\lambda \tau_{1}=\tau_{3}+\tau_{4}$. Write $q\left(\tau_{1}\right)=1+x$, where $1 \neq x$ is of order 3 . Since $q(\lambda)=2.1$, we find that $q\left(\tau_{i}\right)=1+x$, for all $i=1, \ldots, 4$. This is impossible, since by Lemma 1.7.1, we must have $\tau_{1}^{*}=\tau_{i}$ for some $i$, and since $q\left(\tau_{1}^{*}\right)=1+x^{-1}$. This contradiction finishes the proof of the lemma.

Lemma 11.1.11. Suppose that $H$ is simple. Then $|G(H)| \neq 12$.

This discards the possibility that the types $(1,12 ; 2,9),(1,12 ; 3,4)$ and $(1,12 ; 6,1)$ in Lemma 11.1 .1 correspond to a simple Hopf algebra.

Proof. Suppose on the contrary that $|G(H)|=12$.

Claim 11.1.12. $\left|G\left(H^{*}\right)\right|=12$.

Proof. There is a Hopf algebra surjection $H^{*} \rightarrow B$, where $B \simeq$ $k \mathbb{Z}_{3}$. Therefore, $H^{*}$ does not contain any Hopf subalgebra $A$ of dimension 16. Indeed, if this were the case, necessarily $\left(H^{*}\right)^{\text {co } B}=A$, by [NZ]; hence $H$ would not be simple.

Suppose that $\left|G\left(H^{*}\right)\right| \neq 12$. By previous results, $H^{*}$ must be of type $(1,4 ; 2,2 ; 3,4),(1,4 ; 2,11)$ or $(1,4 ; 2,2 ; 6,1)$ as a coalgebra.

Consider first the case where $H^{*}$ is of one of the types $(1,4 ; 2,2 ; 3,4)$ or $(1,4 ; 2,2 ; 6,1)$; so that $H^{*}$ contains a Hopf subalgebra $A$ of dimension 12 , which is of type $(1,4 ; 2,2)$ as a coalgebra. Since $k G(A) \subseteq\left(H^{*}\right)^{\text {co } B}$, and $\operatorname{dim}\left(H^{*}\right)^{\operatorname{co} B}=16$, then $\left(H^{*}\right)^{\operatorname{co} B} \cap A=k G\left(H^{*}\right)$. Let $\pi: H^{*} \rightarrow$ $k^{G(H)}$ be the natural Hopf algebra projection. Then we have $\left(H^{*}\right)^{\operatorname{co} \pi} \subseteq$ $\left(H^{*}\right)^{\operatorname{co} B}$ and $\operatorname{dim}\left(H^{*}\right)^{\operatorname{co} \pi}=4$. Hence, $\left(H^{*}\right)^{\operatorname{co} \pi}=k 1 \oplus W$, for some irreducible left coideal $W$ of dimension 3. (In particular, $H^{*}$ is not of type $(1,4 ; 2,2 ; 6,1)$.) 
Thus $\left(H^{*}\right)^{\operatorname{co} \pi} \cap A=k 1$ and the restriction $\left.\pi\right|_{A}: A \rightarrow k^{G(H)}$ is an isomorphism. This implies that $H$ is a biproduct $H=R \# k G(H)$. Then the claim follows in this case from Proposition 4.4.6.

Suppose next that $H^{*}$ is of type $(1,4 ; 2,11)$. By Proposition 2.1.3, there is a Hopf algebra quotient $H \rightarrow B^{\prime}$ where $B^{\prime}$ is of algebra type $(1,4 ; 2,1)$; so that $\operatorname{dim} H^{\text {co } B^{\prime}}=6$. This implies that $H$ is not of type $(1,12 ; 6,1)$.

By [NZ], any subgroup of order 3 of $G(H)$ is contained in $H^{\text {co } B^{\prime}}$. Hence, $G(H)$ contains a unique subgroup $F$ of order 3 , and $F$ is the unique subgroup of $G(H)$ contained in $H^{\text {co } B^{\prime}}$.

Suppose $H$ is of type $(1,12 ; 3,4)$. Then $H^{\text {co } B^{\prime}}=k F \oplus V$, where $V$ is an irreducible left coideal of $H$ of dimension 3 . Then we have $g V=V=V g$, for all $g \in F$. Let $C$ be the simple subcoalgebra of $H$ containing $V$. By Corollary 3.5.2, $k F$ is normal in $k[C]$.

Besides, since $H^{\text {co } B^{\prime}}$ is normal in $H$, we have $g C g^{-1}=C$, for all $g \in G(H)$. Since $k[C]$ and $G(H)$ necessarily generate $H$ as an algebra, it follows that $k[C]$ is normal in $H$. This discards this type as the coalgebra structure of $H$.

Finally, suppose $H$ is of type $(1,12 ; 2,9)$. Then we must have $H^{\text {co } B^{\prime}}=k F \oplus \oplus_{g \in F} g V$, where $V$ is an irreducible left coideal of $H$ of dimension 2. This is not possible, since $\operatorname{dim} H^{\text {co } B^{\prime}}=6$. Thus $H$ is not type $(1,12 ; 2,9)$ and the proof is complete.

Claim 11.1.12 implies that $H$ is not of type $(1,12 ; 6,1)$ as a coalgebra: indeed, in this case, $H^{\text {co } k^{G\left(H^{*}\right)}} \subseteq k G(H)$ and therefore $k G\left(H^{*}\right)$ is a normal Hopf subalgebra of $H^{*}$.

Since $|G(H)|=\left|G\left(H^{*}\right)\right|=12$, then $H=R \# k \mathbb{Z}_{3}$ is a biproduct. In particular, $G(H)$ contains a unique (normal) subgroup of order 4 . There is in addition a Hopf algebra surjection $H \rightarrow B$, where $\operatorname{dim} B=$ 4. Thus if $F \subseteq G(H)$ is a subgroup of order 3 , then $F \subseteq H^{\operatorname{co} B}$. This shows that $G(H)$ also contains a unique (normal) subgroup of order 3 . Thus $G(H)$ is abelian. This implies that $H$ is not of type $(1,12 ; 3,4)$, in view of Proposition 4.6.1, since in this case $R$ will be a cocommutative coalgebra, by Remark 3.2.7.

It remains to discard the case where $H$ is of type $(1,12 ; 2,9)$. Let $\Gamma \subseteq G(H)$ be the unique subgroup of order 4 . Then there is an irreducible character $\lambda$ of degree 2, such that $\Gamma \cup\{\lambda\}$ spans a standard subalgebra of $R\left(H^{*}\right)$, corresponding to a Hopf subalgebra $A$ of dimension 8.

By $[\mathbf{N Z}]$, we must have $A \subseteq R$. Also, since $R$ is not a Hopf subalgebra, $R$ can contain only one simple subcoalgebra of dimension 4 , which 
implies that this subcoalgebra (and hence all of $A$ ) is stable under the action of $\mathbb{Z}_{3}$ by conjugation.

Hence $B=k\left[A, \mathbb{Z}_{3}\right]$ contains $A$ as a normal Hopf subalgebra, and thus $B \neq H$. Since $\operatorname{dim} B$ is divisible by 3 and $8=\operatorname{dim} A$, we get $\operatorname{dim} B=24$. This implies that $H$ is not simple. The lemma follows.

\subsection{Further reductions}

In this section we further reduce the possibilities for the (co)algebra structure of an eventual simple $H$.

Lemma 11.2.1. Suppose that $H$ is of type $(1,4 ; 2,11)$ as a coalgebra. Then we have

(i) There exists an 8-dimensional non-cocommutative Hopf subalgebra $A_{0} \subseteq H$.

(ii) Assume in addition that $H$ is simple and contains a Hopf subalgebra $A$ of dimension 12. Then neither $A$ nor $A_{0}$ is commutative. In this case, there exists a twist $\phi \in k G(H) \otimes k G(H)$ such that $\left|G\left(H_{\phi}\right)\right|=12,24$ or 48 . In particular, $H_{\phi}$ is not simple.

Proof. Part (i) follows from Proposition 2.1.3. We show part (ii). Note that a Hopf subalgebra of $H$ of dimension 12 is necessarily of coalgebra type $(1,4 ; 2,2)$. Let $B=k\left[A_{0}, A\right]$ be the subalgebra generated by $A_{0}$ and $A$, so that $B$ is a Hopf subalgebra of $H$. Since $8=\operatorname{dim} A_{0}$ divides the dimension of $B$ and also $3 / \operatorname{dim} A / \operatorname{dim} B$, then 24 divides the dimension of $B$. $H$ being simple by assumption, we may assume that $B=H$. In other words, $A_{0}$ and $A$ generate $H$ as an algebra.

Also $G\left(A_{0}\right)=G(A)=G(H)$ is not cyclic.

Suppose that $A$ is commutative. We know that there exists a central group-like element $1 \neq g \in G\left(A_{0}\right)$. Since $A$ is commutative, and $G\left(A_{0}\right)=G(A)$, then $g$ commutes with $A$. Therefore $g$ is central in $k\left[A_{0}, A\right]=H$, contradicting the simplicity of $H$. A similar argument shows that $A_{0}$ cannot be commutative. The last statement of the lemma follows from Proposition 5.2.1.

Lemma 11.2.2. Suppose $H=R \# A$ is a biproduct, where $\operatorname{dim} A=$ 16. Then $H$ is not simple.

Proof. We have $\operatorname{dim} R=3$, thus $R$ is commutative and cocommutative. As a left coideal of $H$, we may assume that $R=k 1 \oplus V$, where $V$ is an irreducible left coideal of dimension 2 .

If $A$ is cocommutative, then the lemma follows from Proposition 4.4.6. So we may assume $A$ is not cocommutative. By Lemma 4.3.3, $\rho(R) \subseteq k G(A) \otimes R$. 
It is not difficult to see that there must exist a normal Hopf subalgebra $B$ of $A$ such that $k G(A) \subseteq B$ and $\operatorname{dim} B=8$. Thus $\rho(R) \subseteq B \otimes R$ and $R \# B$ is a normal Hopf subalgebra (of index 2) of $H$.

Lemma 11.2.3. Suppose that $H$ contains a Hopf subalgebra $A$ with $\operatorname{dim} A=16$. If there is a quotient Hopf algebra $q: H \rightarrow B$, with $\operatorname{dim} B=16$, then $H$ is not simple.

Proof. We have $\operatorname{dim} H^{\mathrm{co} B}=3$. Hence, by Lemma 1.3.4, $A \cap$ $H^{\text {co } B}=k 1$, thus $H=R \# A$ is a biproduct. The lemma follows from Lemma 11.2.2.

LEMma 11.2.4. Suppose that $H$ is of coalgebra type $(1,4 ; 2,2 ; 3,4)$ or $(1,4 ; 2,2 ; 6,1)$. Assume in addition that there is a Hopf algebra quotient $q: H \rightarrow B$, with $\operatorname{dim} B=16$. Then $H$ is not simple.

Proof. We consider first the case where $H$ is of type $(1,4 ; 2,2 ; 6,1)$. Let $\psi$ be the unique irreducible character of degree 6 , and let $\chi_{1}, \chi_{2}$ be the irreducible characters of degree 2 . Then $\chi_{1}, \chi_{2} \in A$, where $A$ is the unique Hopf subalgebra of dimension 12 of $H$; see Remark 11.1.2 (vi). We shall show that $A$ is normal in $H$.

It is not hard to see that

$$
\psi^{2}=\sum_{g \in G(H)} g+2 \chi_{1}+2 \chi_{2}+4 \psi .
$$

Necessarily, we must have $H^{\text {co } B}=k 1 \oplus V$, where $V$ is an irreducible left coideal of $H$ of dimension 2. In particular, $H^{\text {co } B}=A^{\text {co } B}$ and $q(A)$ is a four-dimensional Hopf subalgebra of $B$.

We first claim that $B$ is of type $(1,4 ; 2,3)$ as a coalgebra. To prove this, we consider the possible decompositions of $q(\psi)=\left.\psi\right|_{B^{*}}$ into a sum of irreducible characters in $B$. It follows from Frobenius reciprocity, together with equation (11.2.5), that $q(\psi)=\lambda_{1}+\lambda_{2}+\lambda_{3}$, where $\lambda_{i}$ are pairwise distinct irreducible characters of degree 2 in $B$. Hence $B$ must be of the prescribed coalgebra type.

Therefore, $q(A)=k G(B)$; since this is the unique Hopf subalgebra of dimension 4 in $B$.

On the other hand, it follows from the classification results in $[\mathbf{K}]$, that $k G(B)$ is normal in $B$. For the sake of completeness, we give a proof of this fact in what follows. We may assume that $B$ is not commutative. By $[\mathbf{K}, 3.3], B$ fits into a cocentral abelian extension

$$
1 \rightarrow K \rightarrow B \rightarrow k F \rightarrow 0,
$$

where $F=\left\langle t: t^{2}=1\right\rangle \simeq \mathbb{Z}_{2}$ and $K$ is a commutative Hopf algebra of dimension 8. In particular, $k G(B)$ is contained in $K$ and therefore $G(B)=G(K)$ is central $K$. 
Let $e=\sum_{x \in G(B)} g$. As an algebra, $H$ is a smash product $H=$ $K \# k F$, with respect to an action $\rightarrow: F \times K \rightarrow K$ by Hopf algebra automorphisms. Hence, the action of $t \in F$ permutes the elements of $G(B)$, and therefore $t \rightarrow e=e$.

Note that $H$ is generated as algebra by $K$ and $T:=1 \# t$. It follows from the above discussion that

$$
T e=(t \rightarrow e) \# t=e \# t=T e .
$$

Hence $e \in Z(H)$, which proves the desired fact.

Consider the sequence of surjective Hopf algebra maps

$$
H \stackrel{q}{\longrightarrow} B \stackrel{q^{\prime}}{\longrightarrow} B^{\prime},
$$

where $B^{\prime}=B / B(k G(B))^{+}$. Since $q(A)=k G(B)$, we have $A \subseteq H^{\text {co } q^{\prime} q}$. Thus, by dimension, $A=H^{\operatorname{co} q^{\prime} q}$ and it is a normal Hopf subalgebra, as claimed.

Now consider the case where $H$ is of type $(1,4 ; 2,2 ; 3,4)$. We shall keep the notation in Remark 11.1.2 (v).

For all irreducible character $\psi_{i}$ of degree 3 , we have relations

$$
\psi_{i}^{*} \psi_{i}=1+\chi_{1}+\psi+\psi^{\prime}
$$

and $\chi_{1} \psi_{i}=\psi_{i}+a \psi_{i}$, where $\psi, \psi^{\prime}$ are fixed irreducible of degree 3 , $G\left[\chi_{1}\right]=\{1, a\}$, and $\chi_{1} \in A_{1}$, where $A_{1}$ is the unique six-dimensional Hopf subalgebra of $H$.

Observe first that $\psi^{\prime}=\psi g$, for some $g \in G(H)$. We shall show that $g \in G\left[\chi_{1}\right]$. So that the irreducible characters $1, a, \chi_{1}, \psi, a \psi$, span a standard subalgebra of $R\left(H^{*}\right)$ corresponding to a Hopf subalgebra of dimension 24, whence $H$ is not simple in this case.

We have $H^{\text {co } B}=k 1 \oplus V$, where $V$ is an irreducible left coideal of dimension 2, and necessarily $V \subseteq A_{1}$, since $\operatorname{dim} A_{1}$ does not divide $\operatorname{dim} B$. Hence, $q\left(\chi_{1}\right)=\left.\left(\chi_{1}\right)\right|_{B^{*}}=1+\alpha$, where $1 \neq \alpha \in G(B)$. Since $a \chi_{1}=\chi_{1}$, and $q(a) \neq 1$, then $q(a)=\alpha$.

On the other hand, $m\left(1, q\left(\psi_{i}\right)\right)=m\left(\psi_{i}, \operatorname{Ind}_{B^{*}}^{H^{*}} 1\right)=0$, for all $i=$ $1, \ldots, 4$. The relation $(11.2 .6)$ implies that $m\left(1, q\left(\psi_{i}^{*} \psi_{i}\right)\right)=2$.

This implies that $q(\psi)=\beta+\lambda$ and $q\left(\psi^{\prime}\right)=\beta^{\prime}+\lambda^{\prime}$, for some $\beta, \beta^{\prime} \in G(B)$, and $\lambda, \lambda^{\prime} \in X_{2}(B)$. By Frobenius reciprocity, $\beta, \beta^{\prime}$ and $\alpha$ are pairwise distinct elements of $G(B)$.

Using again equation (11.2.6) for $\psi_{i}=\psi$, we find that $\lambda^{*} \lambda=1+$ $\beta+\beta^{\prime}+\alpha$; hence $\left\{1, \beta, \beta^{\prime}, \alpha\right\}=G\left[\lambda^{*}\right]$ is a subgroup of $G(B)$. Therefore $\beta \alpha=\beta^{\prime}$.

Using Frobenius reciprocity, we have

$$
\psi a=\left(\operatorname{Ind}_{B^{*}}^{H^{*}} \beta\right) a=\operatorname{Ind}_{B^{*}}^{H^{*}}\left(\left.\beta a\right|_{B^{*}}\right)=\operatorname{Ind}_{B^{*}}^{H^{*}}(\beta \alpha)=\operatorname{Ind}_{B^{*}}^{H^{*}}\left(\beta^{\prime}\right)=\psi^{\prime} .
$$


This establishes the claim and finishes the proof of the lemma.

LEMma 11.2.7. Suppose that $H$ contains a Hopf subalgebra $A$ with $\operatorname{dim} A=16$. Then we have:

(i) If $H$ is simple, then $H$ is of type $(1,4 ; 2,11)$ as an algebra, and there exists a normalized 2-cocycle $\phi \in k G\left(H^{*}\right) \otimes k G\left(H^{*}\right)$ such that $\left(H^{*}\right)_{\phi}$ is not simple.

(ii) Assume in addition that $A$ is cocommutative. Then $H$ is not simple.

By part (ii), if $H$ is of type $(1,16 ; 2,8)$ or $(1,16 ; 4,2)$, then $H$ is not simple. Therefore, if $H$ is simple and contains a Hopf subalgebra of dimension $16, H$ is of type $(1,8 ; 2,2 ; 4,2),(1,8 ; 2,10),(1,4 ; 2,3 ; 4,2)$ or $(1,4 ; 2,11)$ as a coalgebra.

Proof. (i) By previous lemmas, $\left|G\left(H^{*}\right)\right| \neq 2,3,6,12$ and there is no Hopf subalgebra $B \subseteq H^{*}$ with $\operatorname{dim} B=16$.

Consider the projection $\pi: H^{*} \rightarrow A^{*}$; we may assume $\left(H^{*}\right)^{\text {co } A^{*}}=$ $k 1 \oplus V$, where $V$ is a left coideal of $H^{*}$ of dimension 2. It follows from Lemma 11.1.1 that $G[\chi] \neq 1$, where $\chi=\chi_{V}$ is the character corresponding to $V$. Then $|G[\chi]|=4$ or 2 .

By Theorem 2.5.1, $H^{*}$ contains a Hopf subalgebra $B$ of dimension $3\left|G\left(H^{*}\right)\right|$. We may assume that $\left|G\left(H^{*}\right)\right|=4$ or 8 , and therefore, $\operatorname{dim} B=12$ or 24 .

Since $H$ is simple, $\operatorname{dim} B=12$, and therefore $\left|G\left(H^{*}\right)\right|=4$. It follows from Lemma 11.2.3 and the results of the previous section, that $H^{*}$ is of type $(1,4 ; 2,2 ; 3,4),(1,4 ; 2,2 ; 6,1)$ or $(1,4 ; 2,11)$ as a coalgebra. In view of Lemma $11.2 .4, H^{*}$ is of type $(1,4 ; 2,11)$ as a coalgebra.

Since $H^{*}$ contains a Hopf subalgebra $A$ of dimension 12, by Lemma 11.2.1, there exists a normalized 2-cocycle $\phi \in k G\left(H^{*}\right) \otimes k G\left(H^{*}\right)$ such that $\left|G\left(H_{\phi}^{*}\right)\right|=12,24$ or 48 . Then $H_{\phi}^{*}$ is not simple. This proves (i).

(ii) We may assume that $|G(H)|=16$ and $H$ is simple. As in the proof of part (i), it follows that $H^{*}$ contains a Hopf subalgebra $B$ of dimension 12. Consider the dual projection $q: H \rightarrow B^{*}$; we have $\operatorname{dim} H^{\text {co } B^{*}}=4$. Also, by a dimension argument using $[\mathbf{N Z}]$, the kernel of the restriction of $q$ to $G(H)$ must be of order 4 . Thus $H^{\text {co } B^{*}} \subseteq$ $k G(H)$ and $q$ is normal. Hence $H$ is not simple in this case.

We summarize the results of this section in the following corollary.

Corollary 11.2.8. If $H$ is simple, the possible coalgebra types for $H$ and $H^{*}$ must be among the following:

$$
\begin{array}{lll}
(1,4 ; 2,2 ; 3,4), & (1,4 ; 2,2 ; 6,1), & (1,4 ; 2,11) \\
(1,4 ; 2,3 ; 4,2), & (1,8 ; 2,2 ; 4,2), & (1,8 ; 2,10) .
\end{array}
$$


Moreover, either $H$ or $H^{*}$ must be of one of the types listed in the first row, and if the type of $H$ is in the second row then the type of $H^{*}$ is $(1,4 ; 2,11)$.

\subsection{Main result up to cocycle twists}

We shall prove in this section that semisimple Hopf algebras of dimension 48 are not simple, up to a cocycle twist.

Lemma 11.3.1. Suppose $H$ is simple. Then $H$ admits no Hopf algebra quotient $H \rightarrow B$, where $B$ is a cocommutative Hopf algebra of dimension 12.

Proof. We shall consider separately the coalgebra types listed in Corollary 11.2.8.

Claim 11.3.2. Suppose $H \rightarrow B$ is a Hopf algebra quotient where $B$ is cocommutative and $\operatorname{dim} B=12$. Then $H^{*}$ is of type $(1,4 ; 2,2 ; 3,4)$ or $(1,4 ; 2,2 ; 6,1)$ as a coalgebra.

Proof. By assumption $B^{*} \subseteq H^{*}$ is a commutative Hopf subalgebra of dimension 12. If $H^{*}$ is not of the prescribed types, then there is a Hopf subalgebra $A \subseteq H^{*}$ of dimension 8 . Since $H$ is simple, we may assume $k\left[A, B^{*}\right]=H$. On the other hand, there exists $1 \neq g \in Z(A) \cap G(A)$. If $g \in B^{*}$, then $g$ is central in $k\left[A, B^{*}\right]=H$ and $H$ is not simple. If $g \notin B^{*}$, then $\left|G\left(H^{*}\right)\right|=8$ and $G\left(B^{*}\right) \subseteq$ $G\left(H^{*}\right)$ is a normal subgroup (of index 2). Then $k G\left(B^{*}\right)$ is normal in $k\left[G\left(H^{*}\right), B^{*}\right]=H$.

Suppose first that $H$ is of type $(1,4 ; 2,2 ; 6,1)$ as a coalgebra. The lemma follows in this case from 1.1, since a cocommutative quotient Hopf algebra $B$ of dimension 12 of $H$ is the same as a commutative Hopf subalgebra $B^{*}$ of index 4 of $H^{*}$, and since $H^{*}$ has an irreducible module of dimension $6>\left[H^{*}: B^{*}\right]$.

Suppose that, as a coalgebra, $H$ is of one of the types

$$
(1,4 ; 2,3 ; 4,2), \quad(1,8 ; 2,2 ; 4,2), \quad(1,8 ; 2,10) .
$$

Then $H$ contains a Hopf subalgebra of dimension 16 and $H^{*}$ is of type $(1,4 ; 2,11)$ as a coalgebra. Then the lemma follows from Lemma 11.2.1.

Suppose next that $H$ is of type $(1,4 ; 2,2 ; 3,4)$. Recall from Remark 11.1.2 (v), that $G(H)$ and the irreducible characters $\chi_{1}$ and $\chi_{2}$ of degree 2 give rise to a Hopf subalgebra $A$ of dimension 12, and for all irreducible characters $\psi_{1}, \ldots, \psi_{4}$ of degree 3 we have $\psi_{i}^{*} \psi_{i}=1+\chi_{1}+\psi+\psi^{\prime}$, where $\psi$ and $\psi^{\prime}$ are fixed elements in $X_{3}$. We also have $\chi_{1}^{2}=1+a+\chi_{1}$, where $\{1, a\}=G\left[\chi_{1}\right] \subseteq G(H)$. 
Suppose that $H$ has a Hopf algebra quotient $q: H \rightarrow B=k G(B)$, where $\operatorname{dim} B=12$. We may assume that $m\left(1, q\left(\psi_{i}\right)\right)=0$, for all $i=$ $1, \ldots, 4$. Otherwise, we would have a decomposition $H^{\text {со } B}=k 1 \oplus W$, where $W$ is an irreducible left coideal of dimension 3, implying that the restriction $q: A \rightarrow B$ is an isomorphism, and thus that $A$ is cocommutative, which is absurd.

We claim that also $m\left(1, q\left(\chi_{1}\right)\right)=0$. If not, since $q\left(\chi_{1}\right)=\left.\chi_{1}\right|_{B^{*}}$, then $m\left(1, q\left(\chi_{1}\right)\right)=1$ by Frobenius reciprocity. Then $q\left(\chi_{1}\right)=1+g$, where $1 \neq g \in G(B)$. The relation $\chi_{1}^{2}=1+a+\chi_{1}$, implies that $q(a)=g$.

Let $1 \neq t \in G(H) \cap H^{\operatorname{co} B}$. Then $q(t)=1$, and $\left(\operatorname{Ind}_{B^{*}}^{H^{*}} 1\right) t=\operatorname{Ind}_{B^{*}}^{H^{*}} 1$; so that $t=a \in G\left[\chi_{1}\right]$. This is a contradiction. Therefore $m\left(1, q\left(\chi_{1}\right)\right)=$ 0 , as claimed.

The relation $\psi_{i}^{*} \psi_{i}=1+\chi_{1}+\psi+\psi^{\prime}$ implies that $m\left(1, q\left(\psi_{i}^{*} \psi_{i}\right)\right)=1$. Write $q\left(\psi_{i}\right)=\sum_{s \in G(B)} n_{s} s$. Then $\sum_{s} n_{s}=\operatorname{deg} \psi_{i}=3$, and $n_{s}=$ $m\left(s, q\left(\psi_{i}\right)\right)=m\left(\psi_{i}, \operatorname{Ind}_{B^{*}}^{H^{*}} s\right) \leq 1$. Hence, the set of all $s \in G(B)$ such that $n_{s} \neq 0$ has at least 3 elements. Now we have

$$
q\left(\psi_{i}^{*} \psi_{i}\right)=q\left(\psi_{i}\right)^{*} q\left(\psi_{i}\right)=\sum_{s, u \in G(B)} n_{s} n_{u} s^{-1} u,
$$

whence $m\left(1, q\left(\psi_{i}^{*} \psi_{i}\right)\right) \geq 3$. This is again a contradiction. Therefore the lemma is established in this case.

It remains to consider the case where $H$ is of type $(1,4 ; 2,11)$. Suppose that there is Hopf algebra quotient $H \rightarrow B$, where $B=k G(B)$ is a cocommutative Hopf algebra of dimension 12. By Corollary 11.2.8, $B^{*}$ is of type $(1,4 ; 2,2)$ as a coalgebra. Hence, $G(B)$ has a normal subgroup of order 3 , and there is a normal Hopf subalgebra $B_{0} \subseteq B$, with $\operatorname{dim} B_{0}=3$.

We may write $H^{\text {co } B}=k 1 \oplus k t \oplus V_{1}$, where $V_{1}$ is an irreducible left coideal of dimension 2, and $1 \neq t \in G(H)$. By Corollary 3.5.2, $t$ is central in $k\left[C_{1}\right]$, where $C_{1}$ is the simple subcoalgebra containing $V_{1}$.

Consider the sequence of surjective Hopf algebra maps

$$
H \stackrel{q}{\longrightarrow} B \stackrel{q^{\prime}}{\longrightarrow} B^{\prime}
$$

where $B^{\prime}=B / B B_{0}^{+}$. We have $\operatorname{dim} H^{\text {co } B^{\prime}}=12$, and there exists an irreducible left coideal $U$ of $H$, not isomorphic to $V_{1}$ such that $m\left(U, H^{\operatorname{co} B^{\prime}}\right)>0$.

Let $\chi \in H$ and $C \subseteq H$ be, respectively, the irreducible character and the simple subcoalgebra corresponding to $U$. Decompose $q(\chi)=$ $\left.\chi\right|_{B^{*}}$ in the form $q(\chi)=g+h$, where $g, h \in G(B)$. Then the restriction of $q$ induces an epimorphism $q: k[C] \rightarrow k\langle g, h\rangle \subseteq k G(B)$. 
Similarly, the restriction of $q^{\prime} q$ induces an epimorphism $q: k[C] \rightarrow$ $k\left\langle g^{\prime}, h^{\prime}\right\rangle \subseteq k G\left(B^{\prime}\right)$, where $q^{\prime} q(\chi)=g^{\prime}+h^{\prime}$. In particular, $g^{\prime}$ and $h^{\prime}$ are the natural projections of $g$ and $h$ in $G\left(B^{\prime}\right)$.

By Frobenius reciprocity, we may assume that $g^{\prime}=1$. Hence

$$
q^{\prime} q(k[C])=k\left\langle h^{\prime}\right\rangle \neq B^{\prime}
$$

because, by Claim 11.3.5, $G\left(B^{\prime}\right)$ is not cyclic. In particular $k[C] \neq H$.

On the other hand, since $g^{\prime}=1$, then $g \in B_{0}$ is of order 3 (because $m(1, q(\chi))=0)$. Hence 3 divides the dimension of $k[C]$. Since $H$ is simple, then $\operatorname{dim} k[C]=12$ or 6 . Also, $\operatorname{dim} k[C]^{\operatorname{co} B}=2$, and it turns out that $1 \neq t$ is a central group-like in $k[C]$.

Since this happens for every irreducible constituent $U$ of $H^{\text {co } B^{\prime}}$, not isomorphic to $V$, it follows that $t$ is central in the Hopf subalgebra generated by all simple subcoalgebras intersecting $H^{\text {co } B^{\prime}}$. This implies that $t$ is central in $H$. This finishes the proof of the lemma.

Lemma 11.3.3. Suppose that $H$ is simple. Then there exists an invertible normalized 2-cocycle $\phi \in k G(\widetilde{H}) \otimes k G(\widetilde{H})$ such that $G\left(\widetilde{H}_{\phi}\right) \simeq$ $G$, where $G$ is the group defined in 5.2. Here, $\widetilde{H}$ is one of the Hopf algebras $H$ or $H^{*}$.

Recall from Chapter 5 that $G$ is the semidirect product $G=F \rtimes \Gamma$, where $F=\left\langle a: a^{3}=1\right\rangle$, and $\Gamma=\left\langle s, t: s^{2}=t^{2}=s t s^{-1} t^{-1}=1\right\rangle$, corresponding to the action by group automorphisms of $\Gamma$ on $F$ defined on generators by s.a $a^{-1}$ and $t . a=a^{-1}$.

Observe that, by Lemma 11.1.1, $\widetilde{H}_{\phi}$ is of one of the types

$$
(1,12 ; 2,9), \quad(1,12 ; 3,4), \quad(1,12 ; 6,1) .
$$

In particular, $\widetilde{H}_{\phi}$ is not simple.

Proof. We shall show that there exist a Hopf subalgebra $A \subseteq \widetilde{H}$ such that $A \simeq \mathcal{A}_{0}$. Thus there is an invertible normalized 2-cocycle $\phi \in k G(\widetilde{H}) \otimes k G(\widetilde{H})$ such that $A_{\phi} \simeq k G$, in view of Proposition 5.2.1. In particular, $\left|G\left(\widetilde{H}_{\phi}\right)\right|$ is divisible by 12 , where $\widetilde{H}$ is either $H$ or $H^{*}$. Thus $\left|G\left(\widetilde{H}_{\phi}\right)\right|=12,24$ or 48 . The following claim implies that indeed $\left|G\left(\widetilde{H}_{\phi}\right)\right|=12$. Hence, $G\left(\widetilde{H}_{\phi}\right) \simeq G$.

Claim 11.3.4. Suppose $\left|G\left(\widetilde{H}_{\phi}\right)\right|=24$ or 48 . Then $H$ is not simple.

Proof. If $\left|G\left(\widetilde{H}_{\phi}\right)\right|=24$, then $k G\left(\widetilde{H}_{\phi}\right)$ is a normal Hopf subalgebra of $\widetilde{H}_{\phi}$. On the other hand we must have $G(\widetilde{H}) \subseteq G\left(\widetilde{H}_{\phi}\right)$. Then $\phi^{-1} \in k G\left(\widetilde{H}_{\phi}\right) \otimes k G\left(\widetilde{H}_{\phi}\right)$. Therefore, in view of Lemma 5.4.1, 
$B=\left(k G\left(\widetilde{H}_{\phi}\right)\right)_{\phi^{-1}}$ is a normal Hopf subalgebra of $\widetilde{H}$ (because it has index 2).

Assume now that $\left|G\left(\widetilde{H}_{\phi}\right)\right|=48$; that is, $\widetilde{H}_{\phi}=k \Gamma$, where $\Gamma=$ $G\left(\widetilde{H}_{\phi}\right)$ is a group of order 48 . For such a group $\Gamma$, either $Z(\Gamma) \neq 1$ or $\Gamma$ contains a normal subgroup of order 16 , that necessarily contains $G(\widetilde{H})$. In any case, $\widetilde{H}$ (and thus $H$ ) is not simple, as claimed.

Since $H$ is simple by assumption, we know that $H$ is of one of the coalgebra types listed in Corollary 11.2.8. Eventually taking $\widetilde{H}=H^{*}$, we may further assume that $H$ has one of the following coalgebra types:

$$
(1,4 ; 2,2 ; 3,4), \quad(1,4 ; 2,2 ; 6,1), \quad(1,4 ; 2,11) .
$$

Claim 11.3.5. The group $G(H)$ is not cyclic.

Proof. If $H$ is of type $(1,4 ; 2,11)$, the claim follows from Lemma 11.2.1 (i). If $H$ is of type $(1,4 ; 2,2 ; 6,1)$, then $H$ contains a simple subcoalgebra $C$ of dimension 36 such that $g C=C=C g$, for all $g \in G(H)$, and the quotient coalgebra $C / C(k G(H))^{+}$is of dimension 9 . If $G(H)$ is cyclic, then any twisted group algebra $k_{\alpha} G(H)$ is cocommutative, but this contradicts Corollary 3.2.5, because $C / C(k G(H))^{+}$cannot have 4 isomorphism classes of simple comodules. Hence the claim follows also in this case.

It remains to consider the type $(1,4 ; 2,2 ; 3,4)$. Suppose on the contrary that $G(H)$ is cyclic. By Corollary 11.2.8, there is a quotient Hopf algebra $q: H \rightarrow Q$ of dimension 4 , so that $\operatorname{dim} H^{\operatorname{co} q}=12$. Keep the notation in Remark 11.1.2 (v). Considering the eventual decomposition of $A^{\operatorname{co} q}, A_{1}^{\operatorname{co} q}$, we distinguish the following two possibilities for $H^{\operatorname{co} q}$ :

(a) $H^{\text {co } q}=k 1 \oplus V \oplus W_{1} \oplus W_{2} \oplus W_{3}$, where $V$ is an irreducible left coideal of dimension 2 , and $W_{i}$ 's are irreducible left coideals of dimension $3, i=1,2,3$.

(b) $H^{\text {co } q}=k 1 \oplus k t \oplus V \oplus V^{\prime} \oplus W_{1} \oplus W_{2}$, where $t \in G(H), V, V^{\prime}$ are irreducible coideals of dimension 2 , and $W_{i}$ 's are irreducible left coideals of dimension $3, i=1,2$.

Note that in this case $t$ is an element of order 2 in $G(H)$, and since this group is cyclic by assumption, necessarily $t=a \in G\left[\chi_{1}\right]$.

Case (a). In this case the restriction of $q$ to $k G(H)$ induces an isomorphism $k G(H) \simeq Q$. Hence $G\left(H^{*}\right)$ contains a cyclic subgroup of order 4 , and by Corollary 11.2.8, we may assume that $H^{*}$ is also of type $(1,4 ; 2,2 ; 3,4)$ as a coalgebra. Hence there is a quotient Hopf algebra $H \rightarrow B$, where $\operatorname{dim} B=12$ and $H^{\text {co } B} \subseteq H^{\text {co } q}$ is a left coideal of dimension 4. Thus we must have a decomposition $H^{\text {co } B}=k 1 \oplus W$, where $W$ is an irreducible left coideal of dimension 3. Let $\psi_{W} \in H$ 
be the character of $W$. Then $g \psi_{W} g^{-1}=\psi_{W}$, for all $g \in G(H)$, and $\left(\psi_{W}\right)^{*}=\psi_{W}$ by Lemma 1.7.1. As in Remark 11.1.2(v) we have a decomposition

$$
\left(\psi_{W}\right)^{*} \psi_{W}=1+\chi_{1}+\psi+\psi^{\prime} .
$$

By Frobenius reciprocity, $m\left(1,\left.\left(\psi_{W}\right)\right|_{B}\right)=1$, hence $\left.\left(\psi_{W}\right)\right|_{B}=1+\lambda$, where $\lambda \in B$ is a (not necessarily irreducible) character such that $m(1, \lambda)=0$; then $m\left(1,\left.\left(\psi_{W}\right)^{*}\left(\psi_{W}\right)\right|_{B}\right) \geq 2$. On the other hand,

$$
\begin{aligned}
m\left(1,\left.\left(\psi_{W}^{*} \psi_{W}\right)\right|_{B}\right) & =1+m\left(1,\left.\left(\chi_{1}\right)\right|_{B}\right)+m\left(1,\left.\psi\right|_{B}\right)+m\left(1,\left.\psi^{\prime}\right|_{B}\right) \\
& =1+m\left(1,\left.\psi\right|_{B}\right)+m\left(1,\left.\psi^{\prime}\right|_{B}\right),
\end{aligned}
$$

and we may assume that $m(1, \psi)>0$, whence $\psi_{W}=\psi$.

This implies that $\psi^{*}=\psi$. Then also $\left(\psi^{\prime}\right)^{*}=\psi^{\prime}$, in view of the relation (11.3.6). Write $\psi^{\prime}=g \psi, 1 \neq g \in G(H)$. Therefore $g^{2}=1$, and since $G(H)$ is cyclic $g=a \in G\left[\chi_{1}\right]$. But this implies that the irreducible characters $G\left[\chi_{1}\right], \chi_{1}, \psi, \psi^{\prime}$ span a standard subalgebra which corresponds to a Hopf subalgebra of $H$ of dimension 24; hence $H$ is not simple in this case.

Case (b). Let $\psi_{i}$ be the character of $W_{i}, i=1,2$. Then $a \psi_{1}=$ $\psi_{2} \neq \psi_{1}$, because $a W_{1}$ is not isomorphic to $W_{1}$ and it is contained in $H^{\text {co } q}$. Moreover, $\psi_{1} \in\left\{\psi, \psi^{\prime}\right\}$, since otherwise, we would have $\psi^{\prime}=a \psi$, implying as before that $H$ contains a Hopf subalgebra of dimension 24 .

Then we may assume that $\psi_{1}=\psi$. Write $\psi^{\prime}=g \psi, 1 \neq g \in G(H)$. If $\psi^{*}=\psi$, then also $\left(\psi^{\prime}\right)^{*}=\psi^{\prime}$ and therefore $g^{2}=1$, whence $g=a$ since $G(H)$ is cyclic. If $\psi^{*}=\psi^{\prime}$, then $\psi^{\prime}=\psi_{2}$, by Lemma 1.7.1. Thus, in any case, $\psi^{\prime}=a \psi$, implying as before, that $H$ contains a Hopf subalgebra of dimension 24. This proves that if $H$ is simple, then the group $G(H)$ is not cyclic, as claimed.

Suppose that $H$ has a Hopf subalgebra $A$ of dimension 12. By Lemma 11.3.1, $H^{*}$ has no cocommutative quotient of dimension 12 . Therefore, $A$ is not commutative. Since $G(H)$ is not cyclic, then $A \simeq \mathcal{A}_{0}$ by $[\mathbf{F}]$. By Proposition 5.2.1 (i), there exists a 2-cocycle $\phi \in k G(A)^{\otimes 2}=k G(H)^{\otimes 2}$ such that $\mathcal{A}_{0} \simeq(k G)_{\phi}$. This establishes the lemma in this case.

We may therefore assume that $H$ is of type $(1,4 ; 2,11)$ and contains no Hopf subalgebra of dimension 12, by Lemma 11.2.1. In particular, $H^{*}$ contains no Hopf subalgebra of dimension 16, by Theorem 2.5.1. We may also assume that $H^{*}$ is not of type $(1,4 ; 2,2 ; 3,4)$ nor $(1,4 ; 2,2 ; 6,1)$, since otherwise we are done by letting $\widetilde{H}=H^{*}$.

By Lemma 11.2.7 (i), we may suppose that $H^{*}$ is also of type $(1,4 ; 2,11)$. Then there is a quotient $q: H \rightarrow B$, where $\operatorname{dim} B=8$ and 
$B$ is not commutative. We may assume that $H^{\text {co } B}=k 1 \oplus k t \oplus V_{1} \oplus V_{2}$, where $t \in G(H)$ and $V_{i}$ is an irreducible coideal of dimension 2, such that $V_{i}$ is not isomorphic to $V_{2}$. Let $\tau_{i} \in X_{2}$ and $C_{i} \subseteq H$ be the irreducible character and simple subcoalgebra corresponding, respectively, to $V_{i}, i=1,2$.

Then $m\left(\tau_{i}, \operatorname{Ind}_{B^{*}}^{H^{*}} 1\right)=1$, and therefore $q\left(\tau_{i}\right)=\left.\tau_{i}\right|_{B^{*}}=1+g_{i}$, where $1 \neq g_{i} \in G(B)$. Moreover, the subgroups $\left\langle g_{1}\right\rangle,\left\langle g_{2}\right\rangle$ are of order at most 4. By construction, $q$ induces by restriction a surjective Hopf algebra $\operatorname{map} q: k\left[C_{i}\right] \rightarrow k\left\langle g_{i}\right\rangle$; in particular, $k\left[C_{i}\right] \neq H$.

If $k\left[C_{i}\right]^{\text {co } B}=k 1 \oplus V_{i}$, respectively $k 1 \oplus k t \oplus V_{1} \oplus V_{2}$, then we have $\operatorname{dim} k\left[C_{i}, G(H)\right]=12$, respectively $\operatorname{dim} k\left[C_{i}\right]=12$. Hence we are done in these cases. If otherwise, $k\left[C_{i}\right]^{\operatorname{co} B}=k 1 \oplus k t \oplus V_{i}$, for all $i=1,2$, Then $t V_{i}=V_{i}=V_{i} t$ and by Corollary 3.5.2, $t$ is central in $k\left[C_{i}\right]$. Thus $t$ is central in $k\left[C_{1}, C_{2}\right]$; and we may assume that $k\left[C_{1}, C_{2}\right] \neq H$. Since 6 divides $\operatorname{dim} k\left[C_{1}, C_{2}\right]$, then we may even assume that $\operatorname{dim} k\left[C_{1}, C_{2}\right]=12$ and we are done in view of Lemma 11.2.1.

\subsection{Main result}

In this section we shall prove our main result in dimension 48. For this we shall first study the normal Hopf subalgebras in a semisimple Hopf algebra $K$ with $|G(K)|=12$. In what follows, we shall denote by $G$ the group considered in Lemma 11.3.3.

THEOREM 11.4.1. Let $H$ be a semisimple Hopf algebra of dimension 48. Then $H$ is not simple.

Proof. By Lemma 11.3.3, we may assume that for $\widetilde{H}=H$ or $H^{*}$, there exists an invertible normalized 2-cocycle $\phi \in k G(\widetilde{H}) \otimes k G(\widetilde{H})$ such that $\widetilde{H}_{\phi}$ is of one of the types

$$
(1,12 ; 2,9), \quad(1,12 ; 3,4), \quad(1,12 ; 6,1),
$$

and $G\left(\widetilde{H}_{\phi}\right) \simeq G$, where $G$ is as in 5.2 .

By Lemma 11.1.11, $\widetilde{H}_{\phi}$ is not simple. Note that $k G(\widetilde{H})=(k G(\widetilde{H}))_{\phi}$ is a cocommutative Hopf subalgebra of dimension 4 of $\widetilde{H}_{\phi}$. Note also that, because $\widetilde{H}=\widetilde{H}_{\phi}$ as algebras, we may assume that $\left|G\left(\widetilde{H}_{\phi}^{*}\right)\right|$ is divisible by 4 . On the other hand, $\phi^{-1} \in k G(\widetilde{H}) \otimes k G(\widetilde{H})$ is a normalized 2-cocycle for $\widetilde{H}_{\phi}$.

Let $K$ be a semisimple Hopf algebra of dimension 48 such that $G(K) \simeq G$. In view of Lemmas 11.4.4, 11.4.5 and 11.4.7 below, at 
least one of the following conditions hold:

(11.4.2) $K$ has a nontrivial central group-like element;

(11.4.3) $G(K)$ is contained in a normal Hopf subalgebra of $K$.

Thus, if $J \in k G(K) \otimes k G(K)$ is an invertible 2-cocycle, the twisted Hopf algebra $K_{J}$ is not simple, by Lemma 5.4.1 and Corollary 5.4.2. This implies the theorem, applied to $K=\widetilde{H}_{\phi}$ and $J=\phi^{-1}$.

In the rest of this section $K$ will be a semisimple Hopf algebra of dimension 48 such that $G(K) \simeq G$; that is, $G(K) \simeq G=F \rtimes \Gamma$, and thus $G$ contains a unique abelian subgroup $M$ of order $6, M=F \times Z$, where $Z=Z(G)$ is of order 2 .

Also, $A \subseteq H$ will be a proper normal Hopf subalgebra. Our aim is to show that at least one of the conditions (11.4.2) or (11.4.3) hold.

Hence, we may assume in what follows that $\operatorname{dim} A \neq 2$. Indeed, by Corollary 1.4.3, if $\operatorname{dim} A=2$, then $A \subseteq k G(K) \cap Z(K)$. Thus, in this case, $K$ verifies condition (11.4.2).

On the other hand, note that we cannot have $A \cap k G(K)$ of dimension 4: this would imply that $A \cap k G(K)=k L$, where $L$ is a normal subgroup of order 4 in $G$ (because $A \cap k G(K)$ is invariant under the adjoint action of $G$ ), which contradicts the structure of $G$.

Hence $\operatorname{dim} A=4,8,16$ are impossible.

Therefore, the possibilities for $\operatorname{dim} A$ are 3, 6, 12 and 24. Moreover, if $\operatorname{dim} A=12$ or 24 , we may assume that $K$ is of type $(1,12 ; 3,4)$. Otherwise, (that is, if $K$ is of type $(1,12 ; 2,9)$ or $(1,12 ; 6,1)$ ), since $\operatorname{dim} A \cap k G(K) \neq 4$, necessarily $G(K) \subseteq A$. See Lemma 6.1.1.

Lemma 11.4.4. Assume $K$ is of type $(1,12 ; 2,9)$ as a coalgebra. Then at least one of the conditions (11.4.2) or (11.4.3) hold.

Proof. By Proposition 2.1.3, $K$ contains a Hopf subalgebra $K_{0}$ of dimension 8. We may assume that $k\left[K_{0}, F\right]=K$; if not, $\operatorname{dim} k\left[K_{0}, F\right]=$ 24 and $k\left[K_{0}, F\right]$ is normal in $K$, whence we are done because $G(K)=$ $G \subseteq k\left[K_{0}, F\right]$.

For all $g \in F, g K_{0} g^{-1}$ is an 8-dimensional Hopf subalgebra of $K$. If $g K_{0} g^{-1}=K_{0}$ for some $1 \neq g \in F$, then $G\left(K_{0}\right)$ would be a normal subgroup of order 4 of $G$, which is a contradiction. Thus the conjugation action of $F$ gives rise to 8-dimensional Hopf subalgebras $K_{0}, K_{1}, K_{2}$ and $G\left(K_{i}\right), 0 \leq i \leq 2$, are distinct subgroups of order 4 of $G$.

We may further assume that $F \subseteq k\left[K_{0}, K_{1}, K_{2}\right]$ and therefore that $k\left[K_{0}, K_{1}, K_{2}\right]=K$. Otherwise $G\left(k\left[K_{0}, K_{1}, K_{2}\right]\right)$ would be of order 4 and since $k\left[K_{0}, K_{1}, K_{2}\right]$ is normal in $K=k\left[K_{0}, K_{1}, K_{2}, F\right]$, then this 
group would be normal in $G(K)$, which is not possible. Note that for each $i=0,1,2, G(K)=\left\langle G\left(K_{i}\right), F\right\rangle$.

We may assume $K$ contains a normal Hopf subalgebra $A$ with $\operatorname{dim} A=3$ or 6 . If $\operatorname{dim} A=3$, then $A=k F$, and in this case, since $k G\left(K_{i}\right)$ is normal in $K_{i}$, then $\operatorname{ad}_{K_{i}} k G(K)=k G(K)$. Thus $k G(K)$ is normal in $K=k\left[K_{0}, K_{1}, K_{2}\right]$. If on the other hand, $\operatorname{dim} A=6$, then because $\operatorname{dim} K / K A^{+}=8$, we have $k F \subseteq A$. Hence $A$ is cocommutative and arguing as before we get that $k G(K)$ is normal in $K$. This finishes the proof of the lemma.

LEMMA 11.4.5. Assume $K$ is of type $(1,12 ; 3,4)$ as a coalgebra. Then at least one of the conditions (11.4.2) or (11.4.3) hold.

Proof. In this case may assume that $\operatorname{dim} A=3,6,12$ or 24 . We may further assume that $K$ contains a normal Hopf subalgebra of dimension 3; that is, $\operatorname{dim} A=3$. To see this, we argue as follows. Suppose that $\operatorname{dim} A=12$. Then $A$ is of type $(1,3 ; 3,1)$ as a coalgebra, and $A$ is commutative. Also, $G(A)$ is the unique (normal) subgroup of order 3 of $G(K)=G$. Thus $k G(A)$ is a 3-dimensional normal Hopf subalgebra in $k[A, G]=K$.

If $\operatorname{dim} A=6$, then $A=k N$, where $N$ is a normal subgroup of order 6 of $G(K)$. Consider the Hopf algebra quotient $K \rightarrow K / K A^{+}$, since $\operatorname{dim} K / K A^{+}=8$, there is a Hopf algebra quotient $K \rightarrow K / K A^{+} \rightarrow B$, where $\operatorname{dim} B=4$. We have $A \subseteq K^{\operatorname{co} B}$, and unless $k G(K)$ is normal in $K, K^{\text {co } B}=A \oplus U \oplus V$, where $U$ and $V$ are irreducible left coideals of $K$ of dimension 3 ; since $N K^{\mathrm{co} B}=K^{\mathrm{co} B}$, we get that $U$ and $V$ are not isomorphic.

In addition, $x V \simeq V \simeq V x$ and $x U \simeq U \simeq U x$, for all $x \in F$, because $G\left[\chi_{U}\right]$ and $G\left[\chi_{V}\right]$ are necessarily of order 3. Let $C_{U}$ and $C_{V}$ be the simple subcoalgebras of $K$ containing $U$ and $V$, respectively. In view of Corollary 3.5.2, $k F$ is normal in $k\left[C_{V}, C_{U}\right]$. Therefore, since it is also normal in $k G(K)$, then $k F$ is normal in $k\left[C_{V}, C_{U}, G(K)\right]=K$.

Finally, if $\operatorname{dim} A=24$, we may assume that $A$ is of type $(1,6 ; 3,2)$ as a coalgebra; see Lemma 6.1.1. In particular, $G(A)$ is a normal subgroup of $G$ of order 6 .

Let $A_{0} \subseteq A$ be a normal Hopf subalgebra. If $\operatorname{dim} A_{0}=12$, then $k F \subseteq Z\left(A_{0}\right)$, and it follows that $k F$ is normal in $K=k\left[A_{0}, G(K)\right]$. If, on the other hand, $A_{0}$ is cocommutative, then $\operatorname{dim} A_{0}=2,3$ or 6 .

In the first case, $A_{0}=k Z$ is the only subgroup of order 2 contained in $G(A)$. Hence $k Z$ is central in $A$ and thus also in $k[A, G(K)]=K$. If $\operatorname{dim} A_{0}=3$, then $A_{0}=k F$ and $k F$ is normal in $k[A, G(K)]=K$. If $\operatorname{dim} A_{0}=6$, then $A_{0}=k N$ is normal in $k[A, G(K)]=K$ and $K$ has a normal Hopf subalgebra of dimension 3, be the above. 
We may therefore assume that $\operatorname{dim} A=3$, as claimed. Then $A=k F$, since $F$ is the only subgroup of order 3 in $G(K)$. In particular, $F=G[\chi]$ for all irreducible character $\chi$ of degree 3. Hence, the quotient Hopf algebra $K / K A^{+}$is cocommutative; see Remark 3.2.7. Say $K / K A^{+} \simeq k N$, where $N$ is a group of order 16 . Since $k F \simeq k^{F}$, then $K$ fits into the abelian extension

$$
1 \rightarrow k^{F} \rightarrow K \rightarrow k N \rightarrow 1
$$

We shall prove in what follows that in this case, $G(K) \cap Z(K) \neq 1$. Hence $K$ satisfies condition (11.4.2).

Consider the matched pair $(F, N)$ associated to the extension, with the actions $\triangleleft: F \times N \rightarrow F$ and $\triangleright: F \times N \rightarrow N$. By [N, Lemma 1.1.7], the exact sequence dual to (11.4.6) gives rise to an exact sequence of groups

$$
1 \rightarrow \widehat{F} \rightarrow G(K) \rightarrow N^{F}
$$

where $N^{F}$ is the subgroup of invariants in $N$ under the action $\triangleright$. Since $|G(K)|=12, N^{F}$ contains a subgroup $N_{1}$ of order 4 of $N$.

On the other hand, the action $\triangleleft$ permutes the set $F \backslash\{1\}$; hence, there is a subgroup $N_{0} \subseteq N$ such that $N_{0}$ acts trivially on $F$ and $\left|N_{0}\right|=8$. We have $N_{0} \cap N_{1} \neq 1$. Let $1 \neq x \in N_{0} \cap N_{1}$.

As a Hopf algebra, $K \simeq k^{F \tau} \#_{\sigma} k N$ is a bicrossed product, corresponding to the actions $k N \otimes k^{F} \rightarrow k^{F}$ obtained from $\triangleleft$, and $k F \otimes k^{N} \rightarrow$ $k^{N}$ obtained from $\triangleright$, and certain 2-cocycles $\sigma: N \times N \rightarrow\left(k^{F}\right)^{\times}$and $\tau: F \times F \rightarrow\left(k^{N}\right)^{\times}$. Moreover, by [N, Lemma 1.2.5], we may assume that $\tau=1$.

The compatibility conditions between $\triangleleft$ and $\triangleright$ imply that $F$ acts on $N_{0}$ by group automorphisms through $\triangleright$. Hence $A_{0}=k^{F} \otimes k N_{0}$ is a Hopf subalgebra of $K$ of dimension 24 .

Since $x \in N_{1}$, then $F$ acts trivially on $x$ and $x \in G(K)$. Also, since $x \in N_{0}$, then $x$ acts trivially in $F$, and thus $x$ commutes with $k^{F}[\mathbf{M}]$.

Since $F$ acts on $N_{0}$ by group automorphisms, this action preserves the center of $N_{0}$. If $N_{0}$ is not abelian, then $\left|Z\left(N_{0}\right)\right|$ is of order 2, and $F$ acts trivially on $Z\left(N_{0}\right)$. Hence we may assume that $x \in Z\left(N_{0}\right)$. Therefore, $1 \neq x \in G\left(A_{0}\right) \cap Z\left(A_{0}\right)$ is an element of order 2. Clearly, the same conclusion holds if $N_{0}$ is abelian, since in this case any $x \in N_{1} \cap N_{0}$ commutes with $N_{0}$.

On the other hand, we may assume that $A_{0}$ is of type $(1,6 ; 3,2)$ as a coalgebra, whence $G\left(A_{0}\right) \cap Z\left(A_{0}\right) \supseteq Z(G(K))$, because $A_{0}$ is normal in $K$. Therefore $Z(G(K))$ is central in $k\left[A_{0}, G(K)\right]=K$. This proves the claim. The proof of the lemma is now complete. 
Lemma 11.4.7. Assume $K$ is of type $(1,12 ; 6,1)$ as a coalgebra. Then at least one of the conditions (11.4.2) or (11.4.3) hold.

Proof. In this case may assume that $\operatorname{dim} A=3$ or 6 . We claim that if $K$ has a Hopf algebra quotient $K \rightarrow B$ with $\operatorname{dim} B=16$, then $k G(K)$ is normal in $K$. Hence the lemma follows when $\operatorname{dim} A=3$.

Observe that the quotient $q: K \rightarrow B$ is necessarily normal, since $k F=K^{\text {co } B}$ by $[\mathbf{N Z}$. In order to establish the claim, we shall follow the lines of the proof of Lemma 11.2.4. We have $q(k G(K))$ is a fourdimensional Hopf subalgebra of $B$. Let $\psi$ be the unique irreducible character of degree 6 . Then we have $\psi^{2}=\sum_{g \in G(K)} g+4 \psi$. This implies, as in the proof of Lemma 11.2.4, that $q(\psi)=\lambda_{1}+\lambda_{2}+\lambda_{3}$, where $\lambda_{i}$ are pairwise distinct irreducible characters of degree 2 in $B$. Hence $B$ must be of type $(1,4 ; 2,3)$ as a coalgebra. Hence, $q(k G(K))=k G(B)$.

We know that $k G(B)$ is normal in $B$. Consider the sequence of surjective Hopf algebra maps $K \stackrel{q}{\rightarrow} B \stackrel{q^{\prime}}{\rightarrow} B^{\prime}$, where $B^{\prime}=B / B(k G(B))^{+}$. Since $q(k G(K))=k G(B)$, then we have $k G(K) \subseteq K^{\text {co } q^{\prime} q}$. Thus, $k G(K)=K^{\mathrm{co} q^{\prime} q}$ is a normal Hopf subalgebra, as claimed.

Suppose next that $\operatorname{dim} A=6$, so that $A=k S$, where $S$ is a subgroup of order 6 in $G$. The quotient Hopf algebra $K / K A^{+}$is cocommutative by Remark 3.2.7. Say $K / K A^{+}=k \Gamma$, where $|\Gamma|=8$. Then $K^{*}=k^{\Gamma} \#_{\sigma} k^{S}$ is a crossed product. Since there exists an irreducible $K^{*}$-module of dimension 6 , then $S=M$ is abelian, by [MW, Proof of Theorem 2.1]. Thus $K$ fits into the abelian extension $1 \rightarrow k^{M} \rightarrow K \rightarrow k N \rightarrow 1$, where $N$ is a group of order 8 such that $K / K A^{+} \simeq k N$. Dualizing, we get an abelian extension $1 \rightarrow k^{N} \rightarrow K^{*} \rightarrow k M \rightarrow 1$. Let $\triangleleft: N \times M \rightarrow N, \triangleright: N \times M \rightarrow M$, be the associated matched pair.

Since the action $\triangleright$ fixes $1 \in M$, and because $N$ is of order 8 , the set of fixed points $M^{N}$ has at least 2 elements. Moreover, by the compatibility condition [M, (4.10)], $M^{N}$ is a subgroup of $M$. It follows also from the formulas $[\mathbf{M},(4.2)$ and (4.5)] for the multiplication and comultiplication of $K$ that the subspace $B:=k^{N} \otimes k\left(M^{N}\right)$ is a Hopf subalgebra of $K^{*}$. If $B=K^{*}$, then then the action $\triangleright$ is trivial. This implies that $k M$ is a central Hopf subalgebra of $K$, which contradicts the assumption on the structure of $G(K)$.

Therefore $\operatorname{dim} B=8\left|M^{N}\right|=16$ or 24 . If $\operatorname{dim} B=16$, the lemma follows from the proof in the case $\operatorname{dim} A=3$. If $\operatorname{dim} B=24$, then $G(K) \cap Z(K) \neq 1$ and the lemma follows as well. 


\section{CHAPTER 12}

\section{Dimension 54}

\subsection{First reduction}

Let $H$ be a nontrivial semisimple Hopf algebra of dimension 54 .

LEMma 12.1.1. The order of $G\left(H^{*}\right)$ is either $2,6,9,18$ or 27 and as an algebra $H$ is of one of the following types:

- $(1,2 ; 2,4 ; 6,1)$,

- $(1,2 ; 2,4 ; 3,4)$,

- $(1,2 ; 2,13)$,

- $(1,6 ; 2,3 ; 6,1)$,

- $(1,6 ; 2,12)$,

- $(1,6 ; 2,3 ; 3,4)$,

- $(1,9 ; 3,1 ; 6,1)$,

- $(1,9 ; 3,5)$,

- $(1,18 ; 2,9)$,

- $(1,18 ; 3,4)$,

- $(1,18 ; 6,1)$,

- $(1,27 ; 3,3)$.

Proof. It follows from 1.1 and Corollary 2.2.3 that $\left|G\left(H^{*}\right)\right| \neq 1$. If $\left|G\left(H^{*}\right)\right|=2$, then the possible algebra types for $H$ other than the prescribed ones are

$$
\begin{aligned}
& (1,2 ; 2,1 ; 4,3),(1,2 ; 3,4 ; 4,1),(1,2 ; 4,1 ; 6,1), \\
& (1,2 ; 2,9 ; 4,1),(1,2 ; 2,5 ; 4,2) .
\end{aligned}
$$

In the last two cases, by Theorems 2.2.1 and 2.4.2 $H$ has a quotient Hopf algebra of algebra types $(1,2 ; 2,9)$ or $(1,2 ; 2,5)$, respectively, which contradicts $[\mathbf{N Z}]$. In the third case, $H$ has one irreducible character $\chi$ of degree 4 , one irreducible character $\psi$ of degree 6 and the remaining two are of degree 1 . The character $\chi$ is necessarily stable under left multiplication by $G\left(H^{*}\right)$ and therefore we have a decomposition $\chi^{2}=\chi \chi^{*}=\epsilon+g+n \chi+m \psi$, implying that $n=2$ and $m=1$. Then we have $1=m=m(\chi, \psi \chi)$ and $\psi \chi=\chi+l \psi, l \in \mathbb{Z}$, which is not possible. The type $(1,2 ; 2,1 ; 4,3)$ is discarded by Lemma 1.2.4. 
Suppose that $H$ is of type $(1,2 ; 3,4 ; 4,1)$. Write $G(H)=\{\epsilon, g\}$ and let $\tau$ be the unique irreducible character of degree 4 . We must have $\tau \tau^{*}=1+g+2 \tau+\psi_{1}+\psi_{2}$, where $\psi_{1} \neq \psi_{2}$ are irreducible characters of degree 3 . Then $m\left(\tau, \psi_{1} \tau\right)=1$ and therefore, $\psi_{1} \tau=\tau+\sum_{j} \psi_{j}$, where $\psi_{j}$ are irreducible of degree 3. Taking degrees we get a contradiction. This discards this possibility.

The possibility $\left|G\left(H^{*}\right)\right|=3$ is discarded using Theorem 2.2.1 and 1.1. As to $\left|G\left(H^{*}\right)\right|=6$, we need to discard the type $(1,6 ; 4,3)$, which is done as follows: let $\chi$ be a irreducible character of degree 4 , and write $\chi \chi^{*}=\sum_{g \in G[\chi]} g+\sum_{i} m_{i} \chi_{i}$, where $\chi_{i}$ are irreducible of degree 4; it turns out that 4 divides the order of $G[\chi]$ which is impossible.

Suppose that $\left|G\left(H^{*}\right)\right|=9$. Then $H$ has no irreducible characters of degree 2 by Theorem 2.2.1 and the only possibilities are the prescribed ones. The rest of the lemma follows also from 1.1.

LEMma 12.1.2. Suppose that $H$ is of type $(1,9 ; 3,5)$ as a coalgebra. Then $H$ is not simple.

Proof. We claim that there exist irreducible characters $\psi \neq \psi^{\prime}$ of degree 3, which commute with $G(H)$, and such that

$$
\psi \psi^{*}=\psi^{\prime}\left(\psi^{\prime}\right)^{*}=\sum_{g \in G(H)} g .
$$

Indeed, the group $G(H)$, being abelian, acts by left multiplication on the set $X_{3}^{\prime}:=\left\{\psi \in X_{3}: g \psi=\psi g, \forall g \in G(H)\right\}$, and we have $\left|X_{3}^{\prime}\right|=2$ or 5 . It follows that there are at least 2 stable elements in $X_{3}^{\prime}$, which therefore satisfy the claimed equations.

The claim implies that there are two subcoalgebras $C_{1}$ and $C_{2}$ of dimension 9, such that $g C_{i}=C_{i}=C_{i} g$, for all $g \in G(H)$. Moreover, we may assume that $k\left[C_{1}, C_{2}\right]=H$ since it is a Hopf subalgebra of dimension bigger than 18, which divides $\operatorname{dim} H$ : if $\operatorname{dim} k\left[C_{1}, C_{2}\right]=27$, then $H$ is not simple by Corollary 1.4.3.

Fix $i=1,2$. By Proposition 3.2.6, $k G(H)$ is normal in $H=$ $k\left[C_{1}, C_{2}\right]$. Therefore $H$ is not simple in this case.

Lemma 12.1.3. Suppose that $H$ is of type $(1,9 ; 3,1 ; 6,1)$ as a coalgebra. Then $H$ is not simple.

Proof. Let $D(H)$ be the Drinfeld double of $H$. By $[\mathbf{N}$, Remark 2.2.4] $\left|G D(H)^{*}\right| \neq 1$. By $[\mathbf{R}]$, the group-like elements in $D(H)^{*}$ are of the form $g \otimes \eta$, where $g \in G(H), \eta \in G\left(H^{*}\right)$, are such that $\eta \otimes g$ is central in $D(H)$. Observe that if the orders of $g$ and $\eta$ are different, say $n=|\eta|<|g|$, then the element $1 \otimes \epsilon \neq g^{n} \otimes \epsilon=(g \otimes \eta)^{n}$ would be such that $\epsilon \otimes g^{n}$ is central in $D(H)$, and a fortiori, $1 \neq g^{n} \in G(H) \cap Z(H)$. 
Then we may assume that there is an element $g \otimes \eta \in G D(H)^{*}$, where $g \in G(H)$ and $\eta \in G\left(H^{*}\right)$ are of the same order. In particular, $\left|G\left(H^{*}\right)\right|$ is divisible by 3 .

On the other hand, the irreducible characters of degree 1 and 3 span a standard subalgebra, which corresponds to a Hopf subalgebra $A$ of dimension 18. Consider the projection $H^{*} \rightarrow A^{*}$; we may assume that $\left(H^{*}\right)^{\text {co } A^{*}}=k 1 \oplus V$, where $V$ is an irreducible left coideal of dimension 2 . Then, by Theorem 2.5.1, $\left|G\left(H^{*}\right)\right|$ is even and there is a quotient Hopf algebra $H \rightarrow B$, with $\operatorname{dim} B=3\left|G\left(H^{*}\right)\right|$. Hence either $\operatorname{dim} B=18$ and necessarily $H^{\operatorname{co} B} \subseteq k G(H)$, or else $H^{*}$ is of type $(1,18 ; 2,9)$ as a coalgebra and $H^{\text {co } k^{G\left(H^{*}\right)}} \subseteq k G(H)$. This implies that $H^{*}$ contains a normal Hopf subalgebra, and hence $H$ is not simple.

REMARK 12.1.4. (i) If $H$ is of type $(1,27 ; 3,3)$, then $H$ is not simple by Corollary 1.4.3.

(ii) Suppose that $H$ is simple. It follows from Lemmas 12.1.1, 12.1.2 and 12.1.3 that there exist subgroups $F \subseteq G(H)$ and $F^{\prime} \subseteq G\left(H^{*}\right)$ such that $|F|=\left|F^{\prime}\right|=2$. We have a projection $q: H \rightarrow \bar{k}^{F^{\prime}}$ such that $\operatorname{dim} H^{\operatorname{co} q}=27$. Hence, $F \cap H^{\operatorname{co} q}=1$ and $H=R \# k F$ is a biproduct. By Proposition 4.6.1, $R$ is not commutative and not cocommutative.

(iii) Suppose that $H$ is of type $(1,6 ; 2,12)$ or $(1,18 ; 2,9)$. Then $H$ is not simple.

Proof. Keep the notation in part (ii). We claim that $G(H)$ contains a central (hence unique) subgroup of order 2, which must stabilize all simple subcoalgebras of dimension 4. This implies, in view of Remark 3.2.7, that the braided Hopf algebra $R$ is a cocommutative coalgebra and we are done.

To prove the claim we distinguish both cases. Suppose first that $H$ is of type $(1,6 ; 2,12)$. Then $G(H)$ is abelian by Proposition 1.2.6, and the claim follows in this case.

In the case of type $(1,18 ; 2,9)$ we argue as follows. First note that by Lemma 2.3.2, $D(H)$ has an irreducible module of dimension 2 . It follows from Theorem 2.2.1 that $G\left(D(H)^{*}\right)$ has an element $g \otimes \eta$ of order 2 or 3 . If $g \otimes \eta$ is of order 2 , we may assume that the center of $G(H)$ has an element of order 2 , as claimed.

Finally suppose that $g \otimes \eta$ is of order 3. By $[\mathbf{N}$, Corollary 2.3.2] there is an exact sequence of Hopf algebras $1 \rightarrow k G \rightarrow D(H) \rightarrow K \rightarrow 1$, and also $G\left(K^{*}\right) \neq 1$ by [N, Remark 2.2.4]; moreover, we may assume that $g \otimes \eta \in G\left(K^{*}\right) \subseteq G\left(D(H)^{*}\right)$. Therefore, by part (ii) in [N , Corollary 2.3.2], we get $\langle\eta, g\rangle=1$.

Consider the natural projection $q: H \rightarrow k^{\langle\eta\rangle}$; we have shown that $\langle g\rangle \subseteq H^{\operatorname{co} q}$. Also, it is clear that $H^{\operatorname{co} q}$ contains every 2-subgoup of 
$G(H)$. Since $H^{\operatorname{co} q}$ is of dimension 18, and we may assume does not coincide with $k G(H)$, then $G(H) \cap H^{\mathrm{co} q}$ is a normal subgroup of order 2 or 6 in $G(H)$. In particular, since we may assume that $1 \neq g \in$ $Z(G(H))$, then the group $G(H) \cap H^{\operatorname{co} q}$ is abelian. But $G(H) \cap H^{\operatorname{co} q}$ contains a unique subgroup of order 2 , then so does $G(H)$. This finishes the proof of the claim.

(iv) By Theorem 2.2.1, since $\operatorname{dim} H$ is not divisible by 4 , for every irreducible character $\chi$ of degree 2 we have $G[\chi] \neq 1$.

(v) If $H$ is of type $(1,2 ; 2,4 ; 6,1),(1,2 ; 2,4 ; 3,4),(1,6 ; 2,3 ; 6,1)$ or $(1,6 ; 2,3 ; 3,4)$ as a coalgebra, then $G(H)$ and $X_{2}$ span a standard subalgebra of $R\left(H^{*}\right)$, which corresponds to a non-cocommutative Hopf subalgebra $A$ of dimension 18. We may thus conclude that if $H$ is simple, then $H$ contains a Hopf subalgebra of dimension 18.

Lemma 12.1.5. Assume that $H$ is of type $(1,2 ; 2,13)$ as a coalgebra. Then $H$ is commutative.

ProOF. By Theorem 4.6.5, we may assume that the order of $G\left(H^{*}\right)$ is odd; thus, by Lemma 12.1.1, $\left|G\left(H^{*}\right)\right|=9$ or 27. Also, by Corollary 4.6.8, we may assume that $G(H) \cap Z(H)=1$ and, in particular, $\left|G\left(H^{*}\right)\right| \neq 27$.

By $\left[\mathbf{N}\right.$, Remark 2.2.4], we have $G\left(D(H)^{*}\right) \neq 1$. Since $\left|G\left(H^{*}\right)\right|$ and $|G(H)|$ are relatively prime, the description of $G\left(D(H)^{*}\right)$ in $[\mathbf{R}]$ implies that there is an isomorphism

$$
G\left(D(H)^{*}\right) \simeq(Z(H) \cap G(H)) \times\left(Z\left(H^{*}\right) \cap G\left(H^{*}\right)\right)=Z\left(H^{*}\right) \cap G\left(H^{*}\right) .
$$

Therefore, $n=\left|G\left(D(H)^{*}\right)\right|=3$ or 9 , and $H^{*}$ contains a central grouplike element of order 3 . Hence $H$ fits into an abelian cocentral extension

$$
1 \rightarrow k^{G} \rightarrow H \rightarrow k \mathbb{Z}_{3} \rightarrow 0
$$

where $G$ is a group of order 18 . Then the extension induces the trivial action $\triangleright: G \times \mathbb{Z}_{3} \rightarrow \mathbb{Z}_{3}$, and an action by group automorphisms $\triangleleft$ : $G \times \mathbb{Z}_{3} \rightarrow G$. This implies that the transpose action $\rightarrow: k \mathbb{Z}_{3} \otimes k^{G} \rightarrow k^{G}$ is by Hopf algebra automorphisms, and therefore that $k G(H)=k \widehat{G}$ is invariant under this action. Hence this action is trivial on $k G(H)$. By [N, Proposition 1.2.6] $H$ is isomorphic as an algebra to the crossed product $H \simeq k^{G} \# \rightarrow k \mathbb{Z}_{3}$. So it turns out that $k G(H)$ is central in $H$. This implies that $H$ is commutative.

Lemma 12.1.6. Assume that $|G(H)|=18$. Then $H$ is not simple.

This discards the following possibilities for the coalgebra type of $H$ :

$$
(1,18 ; 2,9), \quad(1,18 ; 3,4), \quad(1,18 ; 6,1)
$$


PROOF. In view of Remark 12.1.4 (iii) and Lemma 12.1.1, we may assume that $H$ contains no irreducible left coideal of dimension 2. On the other hand, we know from Remark 12.1.4 (v) that there is a quotient Hopf algebra $H \rightarrow B$, where $\operatorname{dim} B=18$. Then necessarily $H^{\text {co } B} \subseteq$ $k G(H)$ is a Hopf subalgebra, and thus $H$ is not simple.

Lemma 12.1.7. Suppose that $H$ is simple. Then $H$ contains a Hopf subalgebra $A \subseteq H$, such that $A \simeq k^{\Gamma}$, where $\Gamma$ is a non-abelian group of order 18. In particular, the dimension of an irreducible left $H$-module is at most 3.

Proof. By Remark 12.1.4 (v) and Lemma 12.1.6 we may assume that $H$ contains a non-cocommutative Hopf subalgebra $A$ of dimension 18. By [M3] there are two isomorphism classes of nontrivial semisimple Hopf algebras of dimension 18, which are dual to each another: $\mathcal{B}_{0}$ and $\mathcal{B}_{1}=\mathcal{B}_{0}{ }^{*}$. We have $\left|G\left(\mathcal{B}_{0}\right)\right|=6$ and $\left|G\left(\mathcal{B}_{1}\right)\right|=9$.

If $A$ is not commutative, and since $G(A) \subseteq G(H)$, we may assume that $|G(A)|=6$. By Remark 12.1.4 (ii), $H=R \# k \mathbb{Z}_{2}$, and since $k \mathbb{Z}_{2} \subseteq A$ and $A$ is not contained in $R=H^{\text {co } \mathbb{Z}_{2}}$, then also $A \simeq R^{\prime} \# k \mathbb{Z}_{2}$. In particular, the order of $G\left(A^{*}\right)$ is also divisible by 2 , which implies that $A$ is commutative as claimed. Finally, applying [AN2, Corollary 3.9] to the inclusion $k^{\Gamma} \subseteq H$, we find that $\operatorname{dim} V \leq 3$, for all irreducible $H$-module $V$.

\subsection{Main result}

After what we have already shown until now, we can conclude that if $H$ is simple, then the possible (co)algebra types for $H$ are $(1,2 ; 2,4 ; 3,4)$ and $(1,6 ; 2,3 ; 3,4)$.

THEOREM 12.2.1. Let $H$ be a semisimple Hopf algebra of dimension 54 over $k$. Then $H$ is not simple.

Proof. Let $A \subseteq H$ be the commutative Hopf subalgebra of dimension 18. Note that $G(H) \subseteq A$.

By Lemma 2.3.2 and Proposition 2.3.1, we have $G\left(D(H)^{*}\right) \neq 1$. Hence, we may assume that there exists a nontrivial one-dimensional Yetter-Drinfeld module for $H$. By Lemma 1.6.1, this has necessarily the form $V_{g, \eta}$, for some $1 \neq g \in G(H)$ and $1 \neq \eta \in G\left(H^{*}\right)$.

Consider the projection $q: H \rightarrow k^{\langle\eta\rangle}$, obtained by transposing the inclusion $k\langle\eta\rangle \subseteq H^{*}$. Since $A$ is commutative, $g^{-1} a g=a$, for all $a \in A^{\operatorname{co} q}$. By Theorem 1.6.4, we must have that $A^{\operatorname{co} q}$ is a Hopf subalgebra of $A$. But this is not possible since $\operatorname{dim} A^{\operatorname{co} q}=9$, implying that $A^{\operatorname{co} q}$ is a cocommutative Hopf subalgebra, while $G(H)$ is of order 2 or 6 . This contradiction finishes the proof of the theorem. 
CHAPTER 13

\section{Dimension 56}

\subsection{First reduction}

Let $H$ be a nontrivial semisimple Hopf algebra of dimension 56 .

LEMMA 13.1.1. The order of $G\left(H^{*}\right)$ is either $4,7,8$ or 28 , and as an algebra $H$ is of one of the following types:

- $(1,4 ; 2,13)$,

- $(1,4 ; 2,9 ; 4,1)$,

- $(1,4 ; 2,5 ; 4,2)$,

- $(1,4 ; 2,1 ; 4,3)$,

- $(1,7 ; 7,1)$,

- $(1,8 ; 4,3)$,

- $(1,8 ; 2,4 ; 4,2)$,

- $(1,8 ; 2,8 ; 4,1)$,

- $(1,8 ; 2,12)$,

- $(1,28 ; 2,7)$.

Proof. We have $G\left(H^{*}\right) \neq 1$ by Corollary 2.2 .3 ; indeed, counting arguments show that the assumption $G\left(H^{*}\right)=1$ implies that $H$ must have an irreducible character of degree 2. Suppose that $\left|G\left(H^{*}\right)\right|=2$, and $H$ is of type $\left(1,2 ; 2, n_{2} ; 3, n_{3} ; \ldots\right)$. If $n_{2} \neq 0$, by Theorems 2.2.1 and 2.4.2, there is a quotient Hopf algebra of dimension $2\left(2 n_{2}+1\right)$; thus $n_{2}=3$ by $[\mathbf{N Z}]$, and using 1.1 we find a contradiction. The possibilities with $n_{2}=0$ are the types $(1,2 ; 3,2 ; 6,1)$ and $(1,2 ; 3,6)$ : these cases are seen to be impossible, after trying to decompose the product $\psi \psi^{*}$ into irreducibles, where $\psi$ is an irreducible character of degree 3.

In the case $\left|G\left(H^{*}\right)\right|=4$, apart from the types listed in the lemma, we have the following possibilities:

$$
(1,4 ; 2,4 ; 6,1),(1,4 ; 2,4 ; 3,4),(1,4 ; 3,4 ; 4,1),(1,4 ; 4,1 ; 6,1) .
$$

In the first two cases, $H$ has a quotient Hopf algebra of dimension 20 , which is impossible. In the third case, $H$ has four degree 1 representations, four irreducible characters $\chi_{1}, \ldots, \chi_{4}$ of degree 3 , and one irreducible character $\psi$ of degree 4 . Then we must have $m\left(\chi_{i}, \psi^{2}\right)=$ $m\left(\chi_{i}, \psi \psi^{*}\right)>0$ for some $i$; also, since $G\left(H^{*}\right)$ must permute transitively 
the degree 3 characters under left or right multiplication and since $g \psi=\psi$, for all $g \in G\left(H^{*}\right)$, then $m\left(\chi_{i}, \psi^{2}\right)>0$ for all $i=1, \ldots, 4$, implying that $m\left(\psi, \chi_{i} \psi\right)=m\left(\chi_{i}, \psi^{2}\right)=1$ for all $i=1, \ldots, 4$. Thus $\chi_{1} \psi=\psi+\sum_{i} m_{i} \chi_{i}$, where $m_{i}$ are non-negative integers not all them equal to zero. Again since $\psi g=\psi$ for all $g$, we find that $m_{i} \neq 0$, for all $i=1, \ldots, 4$. Taking degrees we find a contradiction. This discards the type $(1,4 ; 3,4 ; 4,1)$.

The type $(1,4 ; 4,1 ; 6,1)$ is discarded as follows: let $\chi$ and $\psi$ be the unique irreducible characters of degrees 4 and 6, respectively. Write $\chi^{2}=\sum_{g \in G\left(H^{*}\right)} g+n \chi+m \psi$; thus $m \neq 0$, since this would give a quotient Hopf algebra of dimension 20, which is not possible. Hence $m=2$, and since $m=m(\chi, \psi \chi)$, we have $\psi \chi=2 \chi+l \psi$. Taking degrees we get a contradiction.

It is easy to see that $\left|G\left(H^{*}\right)\right|=14$ is not possible. The rest of the lemma follows from 1.1.

REMARK 13.1.2. (i) If $H$ is of type $(1,28 ; 2,7)$, then $H$ is not simple, by Corollary 1.4.3.

(ii) It follows from Proposition 2.1.3 that, except for the coalgebra type $(1,7 ; 7,1), H$ has a Hopf subalgebra of dimension 8 .

(iii) By [N2], if $G(H) \cap Z(H)=1=G\left(H^{*}\right) \cap Z\left(H^{*}\right)$, then $G(H)$ and $G\left(H^{*}\right)$ are not both of order 8 .

Lemma 13.1.3. Suppose that $H$ is of type $(1,7 ; 7,1)$ as a coalgebra. Then $H$ is not simple.

Proof. We may assume that $H^{*}$ is also of type $(1,7 ; 7,1)$ as a coalgebra; otherwise, there is a quotient Hopf algebra $H \rightarrow B$, where $\operatorname{dim} B=8$ and necessarily $H^{\text {co } B}=k G(H)$ is a normal Hopf subalgebra. Hence $H=R \# k G(H)$ is a biproduct, where $\operatorname{dim} R=8$ and moreover, by Remark 3.2.7, $R$ is cocommutative. Then the lemma follows from Proposition 4.6.1.

LEMma 13.1.4. Suppose that $H$ is simple.

Then $H=R \# A$, where $A$ is a semisimple Hopf algebra of dimension 8 and $R$ is a Yetter-Drinfeld Hopf algebra over $A$ of dimension 7 .

Proof. We may assume that $H$ has a Hopf subalgebra $A$ and a Hopf algebra quotient $q: H \rightarrow B$, such that $\operatorname{dim} A=\operatorname{dim} B=8$.

Since $\operatorname{dim} H^{\text {co } B}=7$, we may assume that $k G(H) \cap H^{\text {co } B}=k 1$. In particular, the lemma follows in the case where $A$ is cocommutative.

If $A$ is not cocommutative, then $A$ contains a unique 4-dimensional simple coalgebra $C$ of $H$. Note that $C$ is not contained in $H^{\text {co } B}$ since $G(H) \subseteq C^{2}$. 
If the restriction $\left.q\right|_{A}: A \rightarrow B$ is an isomorphism, then we are done. Otherwise, $H^{\text {co } B}$ contains a 2-dimensional irreducible left coideal $V$ of $A$. But since $H^{\text {co } B}$ cannot contain $C$, we must have $H^{\text {co } B} \cap A=k 1 \oplus V$. This contradicts Lemma 1.3.4. The proof of the lemma is complete.

\subsection{Main result}

In this section, we apply Lemma 13.1 .4 to show that a semisimple Hopf algebra of dimension 56 is not simple as a Hopf algebra.

TheOREM 13.2.1. Let $H$ be a semisimple Hopf algebra of dimension 56. Then $H$ is not simple.

Proof. Suppose on the contrary that $H$ is simple. Keep the notation in Lemma 13.1.4. After dualizing if necessary, we may assume that $|G(H)|=4$ (see Remark 13.1.2 (ii)); so that $A$ is not cocommutative. As a left coideal of $H, R$ must be of one of the following types:

$$
\text { (1) } k 1 \oplus V_{1} \oplus V_{2} \oplus V_{3}, \quad \text { (2) } k 1 \oplus V \oplus W
$$

where $\operatorname{dim} V_{j}=2=\operatorname{dim} V$, for all $j=1,2,3$, and $\operatorname{dim} W=4$. In particular, the type $(1,4 ; 2,1 ; 4,3)$ is impossible.

Consider first the case (1). By Lemma 4.3.3 $\rho\left(V_{j}\right) \subseteq k G(A) \otimes V_{j}$, for all $j$; thus $\rho(R) \subseteq k G(A) \otimes R$ and therefore $R \# k G(A)$ is a Hopf subalgebra of $H$. This implies that $H$ is not simple, since $G(A)$ has index 2 in $A$.

Suppose finally that $R$ is as in case (2). By Lemma 4.3.3 $\rho(V) \subseteq$ $k G(A) \otimes V$. Moreover, $k G(A) . V \subseteq V$, since $g V g^{-1}$ is a 2-dimensional irreducible left coideal of $H$ contained in $R$, for all $g \in G(H)$.

By Proposition 4.2.1, $V$ is an $A$-subcomodule subcoalgebra of $R$. Then, by Lemma 4.3.1, $B=k[V] \# k G(A)$ is a Hopf subalgebra of $H$. Since $\operatorname{dim} B$ is divisible by 4 and $\operatorname{dim} k[V] \geq 3$, we must have $\operatorname{dim} B=28$ and $k V=R$. As before, we get that $R \# k G(A)$ is a Hopf subalgebra of index 2 in $H$, and $H$ is not simple. 


\section{APPENDIX A}

\section{Drinfeld Double of $H_{8}$}

We shall denote by $H_{8}$ the unique nontrivial 8-dimensional semisimple Hopf algebra over $k[\mathbf{K P}, \mathbf{M} 4]$. We shall use the notation $D_{4}$ and $Q$ to indicate, respectively, the dihedral and quaternionic groups of order 8. For a Hopf algebra $A, D(A)$ denotes the Drinfeld double of $A$.

By [M4] the only non-commutative semisimple Hopf algebras of dimension 8 are $H_{8}, k D_{4}$ and $k Q$.

\section{A.1. Structure of $D\left(H_{8}\right)$}

Tambara and Yamagami show in $[\mathbf{T Y}]$ that the categories of representations of these three Hopf algebras are not equivalent as monoidal categories. The comparison of Schur indicators implies that the representation theory of $H_{8}$ is in some sense closer to that of $k D$ than to that of $k Q$; see [Mo3]. On the other hand, note that $H_{8}$ fits into an exact sequence $1 \rightarrow k^{\Gamma} \rightarrow H_{8} \rightarrow k F \rightarrow 1$, where $\Gamma \simeq \mathbb{Z}_{2} \times \mathbb{Z}_{2}$ and $F \simeq \mathbb{Z}_{2}$, such that the associated product group associated to the extension is $D_{4}$.

The main results in this appendix are the following:

Theorem A.1.1. (i) $D\left(H_{8}\right)$ fits into an abelian central extension

$$
0 \rightarrow k^{G} \rightarrow D\left(H_{8}\right) \rightarrow k G \rightarrow 1,
$$

where $G=G\left(D\left(H_{8}\right)^{*}\right) \simeq \mathbb{Z}_{2} \times \mathbb{Z}_{2} \times \mathbb{Z}_{2}$.

(ii) $D\left(H_{8}\right)$ is of type $(1,8 ; 2,14)$ as an algebra, and as a coalgebra it is of type $(1,16 ; 2,8 ; 4,1)$.

THEOREM A.1.2. $D\left(H_{8}\right)$ has no quotient Hopf algebra isomorphic to $k Q$.

Observe that since $H_{8}$ admits quasitriangular structures [Su], then $D\left(H_{8}\right)$ also has quotient Hopf algebras isomorphic to $H_{8}$.

REMARK A.1.3. Let $G$ be a finite group. Then $D(G)$ is a semidirect product $D(G)=k^{G} \rtimes k G$, with respect to the action comming from the adjoint action of $G$ on itself. Hence, the irreducible representations of $D(G)$, viewed as irreducible Yetter-Drinfeld modules over $k G$, are classified by the modules $V_{g, \rho}:=k G \otimes_{Z_{G}(g)} \rho$, where $g$ runs over a 
system of representatives of the conjugacy classes in $G$ and $\rho$ runs over the irreducible representations of the centralizer $Z_{G}(g)$.

This implies that if $|G|=p^{3}, p$ prime, then $D(G)$ has exactly $p^{3}$ one-dimensional representations and the remaining irreducible representations are of dimension $p$; that is, $D(G)$ is of type $\left(1, p^{3} ; p, p\left(p^{3}-1\right)\right)$ as an algebra.

In particular, the Drinfeld doubles of the three non-commutative 8dimensional semisimple Hopf algebras have the same algebra structure.

\section{A.2. Proof of Theorem A.1.1}

Let $A$ be a finite-dimensional Hopf algebra and let $g \in G(A), \eta \in$ $G\left(A^{*}\right)$. Let $V_{g, \eta}$ denote the vector space $k 1$ endowed with the action $h .1=\eta(h) 1, h \in H$, and the coaction $1 \mapsto g \otimes 1$.

By Lemma 1.6.1, the one-dimensional Yetter-Drinfeld modules over $A$ are exactly of the form $V_{g, \eta}$, where $g \in G(A)$ and $\eta \in G\left(A^{*}\right)$ are such that $(\eta \rightarrow h) g=g(h<\eta)$, for all $h \in A$.

Note that if the condition $(\eta \rightarrow h) g=g(h<\eta)$ holds for all $h$ in a set of generators of $A$, then it holds for all $h \in A$.

It turns out that the elements of the form $\eta \otimes g$, where $g$ and $\eta$ satisfy the condition in Lemma 1.6 .1 are exactly the central group-like elements in $D(A)[\mathbf{R}]$. In particular, $V_{g, \epsilon}$ (respectively, $\left.V_{1, \eta}\right)$ is a YetterDrinfeld module if and only if $g \in Z(A)$ (respectively, $\eta \in Z\left(A^{*}\right)$ ).

As in $[\mathbf{M} 4], H_{8}$ can be presented by generators $x, y, z$ with relations

$$
\begin{aligned}
& x^{2}=y^{2}=1 \\
& x y=y x, \quad z x=y z, \quad z y=x z, \\
& z^{2}=\frac{1}{2}(1+x+y-x y) .
\end{aligned}
$$

The coalgebra structure is determined by

$$
\begin{aligned}
& \Delta(x)=x \otimes x, \quad \Delta(y)=y \otimes y, \\
& \Delta(z)=\frac{1}{2}((1+y) \otimes 1+(1-y) \otimes x)(z \otimes z) .
\end{aligned}
$$

In particular, $z \in H_{8}^{\times}, \epsilon(z)=1$ and $\mathcal{S}(z)=z^{-1}$. We have in addition $H_{8} \simeq H_{8}^{*}, G\left(H_{8}\right)=\{1, x, y, x y\} \simeq \mathbb{Z}_{2} \times \mathbb{Z}_{2}$, and $G\left(H_{8}\right) \cap$ $Z\left(H_{8}\right)=\{1, x y\}$.

LEMmA A.2.1. Let $g \in G\left(H_{8}\right) \backslash Z\left(H_{8}\right)$ and $\eta \in G\left(H_{8}^{*}\right) \backslash Z\left(H_{8}^{*}\right)$. Then $V_{g, \eta}$ is a Yetter-Drinfeld module of $H_{8}$. 
This implies that $D\left(H_{8}\right)$ has exactly 8 distinct one-dimensional representations. In other words, we have

$$
G\left(D\left(H_{8}\right)^{*}\right)=\langle x y \otimes \epsilon\rangle \oplus\langle 1 \otimes \alpha \beta\rangle \oplus\langle x \otimes \alpha\rangle,
$$

where $G\left(H_{8}^{*}\right)=\{\epsilon, \alpha, \beta, \alpha \beta\}$ and $G\left(H_{8}^{*}\right) \cap Z\left(H_{8}^{*}\right)=\{\epsilon, \alpha \beta\}$.

Proof. We shall show that if $1, x y \neq g$, then every element $\eta \in$ $G\left(H_{8}^{*}\right) \backslash Z\left(H_{8}^{*}\right)$ satisfies the condition in Lemma 1.6.1.

Notice that for any one-dimensional representation $\eta: H_{8} \rightarrow k$ we must have $\eta(x)=\eta(y)$, because of the relation $z x=y z$ and the fact that $z \in H_{8}^{\times}$. Moreover, if $\eta$ is not central in $H_{8}^{*}$, then $\eta(x)=$ $\eta(y)=-1$ : indeed, if $\left.\eta\right|_{G(H)}=1$, then $k\langle\eta\rangle=\left(H_{8}^{*}\right)^{\operatorname{co} G\left(H_{8}\right)}$ and thus $\eta \in Z\left(H_{8}^{*}\right)$.

Also, it is enough to see that the condition in Lemma 1.6.1 is satisfied for $h=z$, since it is always satisfied for $h=x, y$ and these generate $H_{8}$ as an algebra.

We compute

$$
\begin{aligned}
& \eta \rightarrow z=\eta\left(z_{2}\right) z_{1}=\frac{1}{2}(1+y+\eta(x)(1-y)) \eta(z) z, \\
& z<\eta=\eta\left(z_{1}\right) z_{2}=\frac{1}{2}(\eta(1+y) 1+\eta(1-y) x) \eta(z) z .
\end{aligned}
$$

Replacing in this identities $g=y$, the condition $(\eta \rightarrow z) y=y(z<$ $\eta)$ is equivalent to the equation $x y+x+\eta(x)(x-x y)=\eta(1+y) y+$ $\eta(1-y) x y$. This is always satisfied for $\eta \in G\left(H_{8}^{*}\right) \backslash Z\left(H_{8}^{*}\right)$.

The argument for $g=x$ is similar.

Corollary A.2.5. $G\left(D\left(H_{8}\right)^{*}\right) \simeq \mathbb{Z}_{2} \times \mathbb{Z}_{2} \times \mathbb{Z}_{2}$.

Proof. We have $\left|G\left(D\left(H_{8}\right)^{*}\right)\right|=8$ and $G\left(D\left(H_{8}\right)^{*}\right)$ is isomorphic to a subgroup of $G\left(D\left(H_{8}\right)\right)=G\left(H_{8}^{*}\right) \times G\left(H_{8}\right)$.

Proof of Theorem A.1.1. (i) In virtue of [N, Corollary 2.3.2] and Corollary A.2.5, there is a central extension

$$
0 \longrightarrow k G \stackrel{\iota}{\longrightarrow} D\left(H_{8}\right) \stackrel{\pi}{\longrightarrow} K \longrightarrow 1,
$$

where $G \simeq G\left(D\left(H_{8}\right)^{*}\right) \simeq \mathbb{Z}_{2} \times \mathbb{Z}_{2} \times \mathbb{Z}_{2}$, and the map $\iota$ is determined by $\iota(g \otimes \eta)=\eta \otimes g$. In particular, $\operatorname{dim} K=8$.

We have $k G \simeq k^{G}$. Identify $K$ with a Hopf subalgebra of $D\left(H_{8}\right)^{*}$. Part (i) will be established if we show that $G\left(K^{*}\right)=G$. To see this, we observe that a group-like element $g \otimes \eta$ belongs to $G\left(K^{*}\right)$ exactly when it is a one dimensional representation of $D\left(H_{8}\right)$ which factorizes through $K$; that is, $g \otimes \eta$ belongs to $G\left(K^{*}\right)$ if and only if

$$
\left\langle\eta, g^{\prime}\right\rangle\left\langle\eta^{\prime}, g\right\rangle=1
$$


for all group-like elements $g^{\prime} \otimes \eta^{\prime} \in G\left(D\left(H_{8}\right)^{*}\right)$. See [N, Corollary 2.3.2]. Finally, using the description of the elements in $G\left(D\left(H_{8}\right)^{*}\right)$ given in (A.2.2), one sees that $G\left(K^{*}\right)=G$, thus proving part (i).

(ii) As a coalgebra, $D\left(H_{8}\right)$ is a tensor product: $D\left(H_{8}\right)=\left(H_{8}^{*}\right)^{\text {cop }} \otimes$ $H_{8}$. This proves the statement corresponding to the coalgebra structure.

Combining part (i) with the description in [KMM, Theorem 3.3] (the action being trivial in our situation), we find that the simple $D\left(H_{8}\right)$-modules are of the form $p_{g} \otimes V$, where $g \in G$ and $V$ is an irreducible $k_{\sigma_{g}} G$-irreducible module, for some 2-cocycle $\sigma_{g}: G \times G \rightarrow k^{\times}$. The dimensions of the irreducible $k_{\sigma_{g}} G$-modules are either 1 or 2 (since they divide the order of $G$ and their square is less than 8). This finishes the proof of (ii).

\section{A.3. Proof of Theorem A.1.2}

Suppose that $\pi: D\left(H_{8}\right) \rightarrow k M$ is a surjective Hopf algebra map, where $M$ is a group of order 8 . Then $k M=\pi\left(H_{8}^{*}\right) \pi\left(H_{8}\right)$ and $\operatorname{dim} \pi\left(H_{8}^{*}\right)$ and $\operatorname{dim} \pi\left(H_{8}\right)$ divide 4 (because $H_{8} \simeq H_{8}^{*}$ is not isomorphic to $k M$ ). Then some of them, say $\pi\left(H_{8}\right)$ is of dimension 4. Then $\pi\left(H_{8}\right) \simeq$ $\mathbb{Z}_{2} \times \mathbb{Z}_{2}$, since $H_{8}^{*}$ has no Hopf subalgebra isomorphic to $\mathbb{Z}_{4}$. Therefore $M \neq Q$. 


\section{Bibliography}

[A] N. Andruskiewitsch, Notes on extensions of Hopf algebras, Canad. J. Math. 48 (1996), 3-42.

[A2] N. Andruskiewitsch, About finite dimensional Hopf algebras, Contemp. Math. 294 (2002), 1-57.

[AN] N. Andruskiewitsch and S. Natale, Examples of self-dual Hopf algebras, J. Math. Sci. Univ. Tokyo 6 (1999), 181-215.

[AN2] N. Andruskiewitsch and S. Natale, Harmonic analysis on semisimple Hopf algebras, Algebra i Analiz 12 (2000), 3-27.

[AN3] N. Andruskiewitsch and S. Natale, Braided Hopf algebras arising from matched pairs of groups, J. Pure Appl. Algebra 132 (2003), 119-149.

[CR] C. Curtis and I. Reiner, Methods of Representation Theory Vol. I, Wiley Interscience, New York, 1990.

[EG] P. Etingof and S. Gelaki, Semisimple Hopf algebras of dimension $p q$ are trivial, J. Algebra 210 (1998), 664-669.

[EG2] P. Etingof and S. Gelaki, Some properties of finite dimensional semisimple Hopf algebras, Math. Res. Lett. 5 (1998), 551-561.

[EG3] P. Etingof and S. Gelaki, The representation theory of co-triangular semisimple Hopf algebras, Int. Math. Res. Not. 7 (1999), 387-394.

[EG4] P. Etingof and S. Gelaki, The classification of triangular semisimple and cosemisimple Hopf algebras over an algebraically closed field, Int. Math. Res. Not. 5 (2000), 223-234.

[EG5] P. Etingof and S. Gelaki, Isocategorical groups, Int. Math. Res. Not. 2 (2001), $59-76$.

[F] N. Fukuda, Semisimple Hopf algebras of dimension 12, Tsukuba J. Math. 21 (1997), 43-54.

[GN] C. Galindo and S. Natale, Simple Hopf algebras and deformations of finite groups, preprint math.QA/0608734 (2006).

[G] S. Gelaki, Quantum groups of dimension $p q^{2}$, Israel J. Math. 102 (1997), $227-267$.

[GW] S. Gelaki and S. Westreich, On semisimple Hopf algebras of dimension pq, Proc. Am. Math. Soc. 128 (2000), 39-47. (Corrigendum: Proc. Am. Math. Soc. 128 (2000), 2829-2831.)

[IK] M. Izumi and H. Kosaki, Kac algebras arising from composition of subfactors: general theory and classification, Mem. Amer. Math. Soc. 158, 750, (2002).

[Ka] G. Kac, Extensions of groups to ring groups, Math. USSR. Sb. 5 (1968), 451-474.

[Ka2] G. Kac, Certain arithmetic properties of ring groups, Functional Anal. Appl. 6 (1972), 158-160. 


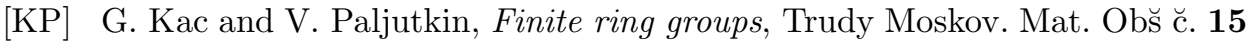
(1966) 224-261.

[K] Y. Kashina, Classification of semisimple Hopf algebras of dimension 16, J. Algebra 232 (2000), 617-663.

[K2] Y. Kashina, Some results on Hopf algebras of Frobenius type, Math. Appl., Dordr. 555 (2003), 85-104.

$[\mathrm{KMM}]$ Y. Kashina, G. Mason and S. Montgomery, Computing the Frobenius-Schur indicator for abelian extensions of Hopf algebras, J. Algebra 251 (2002), 888913.

$[\mathrm{KM}]$ T. Kobayashi and A. Masuoka, A result extended from groups to Hopf algebras, Commun. Algebra 25 (1997), 1169-1197.

[LR] R. Larson and D. Radford, Semisimple cosemisimple Hopf algebras, Amer. J. Math. 110 (1988), 187-195.

[LR2] R. Larson and D. Radford, Finite dimensional cosemisimple Hopf algebras in characteristic 0 are semisimple, J. Algebra 117 (1988), 267-289.

[Mj] S. Majid, Crossed products by braided groups and bosonization, J. Algebra 163 (1994), 165-190.

[Mj2] S. Majid, Foundations of Quantum Group Theory, Cambridge Univ. Press (1995).

[M] A. Masuoka, Extensions of Hopf algebras, Trabajos de Matemática 41/99, FaMAF (1999).

[M2] A. Masuoka, Freeness of Hopf algebras over coideal subalgebras, Commun. Algebra 20 (1992) 1353-1373.

[M3] A. Masuoka, Some further classification results on semisimple Hopf algebras, Commun. Algebra 24 (1996), 307-329.

[M4] A. Masuoka, Semisimple Hopf algebras of dimension 6, 8, Israel J. Math. 92 (1995), 361-373.

[M5] A. Masuoka, Cocycle deformations and Galois objects for some co-semisimple Hopf algebras of finite dimension, Contemp. Math. 267 (2000), 195-214.

[M6] A. Masuoka, The $p^{n}$-th Theorem for Hopf algebras, Proc. Amer. Math. Soc. 124 (1996), 187-195.

[M7] A. Masuoka, Self dual Hopf algebras of dimension $p^{3}$ obtained by extension, J. Algebra 178 (1995), 791-806.

[M8] A. Masuoka, Semisimple Hopf algebras of dimension 2p, Comm. Algebra 23 (1995), 1931-1940.

[M9] A. Masuoka, Coalgebras actions on Azumaya algebras, Tsukuba J. Math. 14 (1990), 107-112.

[M10] A. Masuoka, Hopf algebra extensions and cohomology, Math. Sci. Res. Inst. Publ. 43 (2002), 167-209.

[Mo] S. Montgomery, Hopf Algebras and their Actions on Rings, CMBS Reg. Conf. Ser. in Math. 82, Amer. Math. Soc., 1993.

[Mo1] S. Montgomery, Classifying finite dimensional semisimple Hopf algebras, Contemp. Math. 229 (1998), 265-279.

[Mo2] S. Montgomery, Indecomposable coalgebras, simple comodules and poin-ted Hopf algebras, Proc. Amer. Math. Soc. 123 (1995), 2343-2351.

[Mo3] S. Montgomery, Representation theory of semisimple Hopf algebras, Algebra - representation theory (Constanta, 2000), 189-218, NATO Sci. Ser. II Math. Phys. Chem. 28, Kluwer Acad. Publ., Dordrecht, 2001. 
[MW] S. Montgomery and S. Whiterspoon, Irreducible representations of crossed products, J. Pure Appl. Algebra 129 (1998), 315-326.

[N] S. Natale, On semisimple Hopf algebras of dimension $p q^{2}$, J. Algebra 221 (1999), 242-278.

[N2] S. Natale, On semisimple Hopf algebras of dimension $p q^{2}$, II, Algebr. Represent. Theory 5 (3), (2001), 277-291.

[N3] S. Natale, On semisimple Hopf algebras of dimension $p q^{r}$, Algebr. Represent. Theory 7 (2) (2004), 173-188.

[N4] S. Natale, On semisimple Hopf algebras of low dimension, AMA Algebra Montp. Announc. 01 (2003).

[N5] S. Natale, On group theoretical Hopf algebras and exact factorizations of finite groups, J. Algebra 270 (1) (2003), 199-211. Preprint math.QA/0208054.

[NR] W. Nichols and M. Richmond, The Grothendieck group of a Hopf algebra, J. Pure Appl. Algebra 106 (1996), 297-306.

[NZ] W. Nichols and M. Zoeller, A Hopf algebra freeness Theorem, Amer. J. Math. 111 (1989), 381-385.

[Nk] D. Nikshych, $K_{0}$-rings and twisting of finite-dimensional semisimple Hopf algebras, Commun. Algebra 26 (1998), 321-342. (Corrigendum: Commun. Algebra 26 (1998), 2019.)

[R] D. Radford, Minimal quasitriangular Hopf algebras, J. Algebra 157 (1993), $285-315$.

[R2] D. Radford, The structure of Hopf algebras with a projection, J. Algebra 92 (1985), 322-347.

[Sb] P. Schauenburg, On the braiding on a Hopf algebra in a braided category, New York J. Math. 4 (1998), 259-263.

[Sc] H.-J. Schneider, Lectures on Hopf algebras, Trabajos de Matemática 31/95, FaMAF (1995).

[Sc2] H.-J. Schneider, Normal basis and transitivity of crossed products for Hopf algebras, J. Algebra 152 (1992), 289-312.

[Sc3] H.-J. Schneider, Principal homogeneous spaces for arbitrary Hopf algebras, Israel J. Math. 72 (1990), 167-195.

[So] Y. Sommerhäuser, Yetter-Drinfel'd Hopf algebras over groups of prime order, Lecture Notes in Math. 1789 (2002), Springer-Verlag.

[Su] S. Suzuki, A family of braided cosemisimple Hopf algebras of finite dimension, Tsukuba J. Math. 22 (1998), 1-29.

[T] D. Tambara, Representation of tensor categories with fusion rules of selfduality for finite abelian groups, Israel J. Math. 118 (2000), 29-60.

[TY] D. Tambara and S. Yamagami, Tensor categories with fusion rules of selfduality for finite abelian groups, J. Algebra 209 (1998), 29-60.

[ZS] S. Zhu, On finite dimensional Hopf algebras, Commun. Algebra 21 (1993), 3871-3885.

[Z] Y. Zhu, Hopf algebras of prime dimension, Int. Math. Res. Not. 1 (1994), $53-59$.

[Z2] Y. Zhu, A commuting pair in Hopf algebras, Proc. Amer. Math. Soc. 125 (1997), 2847-2851. 